University of Louisville

ThinkIR: The University of Louisville's Institutional Repository

Electronic Theses and Dissertations

8-2009

\title{
Ultrakinetic features of anime texts: revisioning composition theory and exploring visual rhetoric pedagogy.
}

Karen Ware 1973-

University of Louisville

Follow this and additional works at: https://ir.library.louisville.edu/etd

\section{Recommended Citation}

Ware, Karen 1973-, "Ultrakinetic features of anime texts: revisioning composition theory and exploring visual rhetoric pedagogy." (2009). Electronic Theses and Dissertations. Paper 1530.

https://doi.org/10.18297/etd/1530

This Doctoral Dissertation is brought to you for free and open access by ThinkIR: The University of Louisville's Institutional Repository. It has been accepted for inclusion in Electronic Theses and Dissertations by an authorized administrator of ThinkIR: The University of Louisville's Institutional Repository. This title appears here courtesy of the author, who has retained all other copyrights. For more information, please contact thinkir@louisville.edu. 
ULTRAKINETIC FEATURES OF ANIME TEXTS:

REVISIONING COMPOSITION THEORY

AND EXPLORING VISUAL RHETORIC PEDAGOGY

\title{
By
}

Karen O'Connell Ware

B.A., Spalding University, 1994

M.A., University of Louisville, 2001

\author{
A Dissertation \\ Submitted to the Faculty of the \\ Graduate School of the University of Louisville \\ in Partial Fulfillment of the Requirements \\ for the Degree of
}

Doctor of Philosophy

Department of English University of Louisville

Louisville, Kentucky

August 2009 
Copyright 2009 by Karen O'Connell Ware

All rights reserved 


\title{
ULTRAKINETIC FEATURES OF ANIME TEXTS: REVISIONING COMPOSITION THEORY AND EXPLORING VISUAL RHETORIC PEDAGOGY
}

\author{
By \\ Karen O'Connell Ware \\ B.A., Spalding University, 1994 \\ M.A., University of Louisville, 2001 \\ A Dissertation Approved on
}

August 11, 2009

by the following Dissertation Committee:

Dissertation Director 


\section{DEDICATION}

For Kyle,

through all things.

And for Paul, who would be proud. 


\section{ACKNOWLEDGMENTS}

If I were to thank everyone who has supported the process of writing this dissertation in a manner proportional to the assistance, this page would rival the length of the dissertation. The time my readers have afforded me is beyond the call of duty and I am grateful for their effort and thoughtfulness. Dr. David Anderson committed to the project early and stayed the course, despite how long the timeline became. Dr. Dennis Hall was willing to step in with his guidance and has provided a keen eye. Dr. Cynthia Selfe gave me an amazing opportunity to assist her in teaching a course at UofL and made the leap to hang on for the ride of this dissertation. Dr. Pam Takayoshi has been a nurturing resource in my academic life from the moment I met her. The title "Director" does not encompass the role Dr. Brian Huot has played; he is a mentor and an inspiration. His drive to challenge me has been tireless and I am better for it. I thank Sherleen Sisney for being an extraordinary person and an unusual "boss" who is personally loyal and supportive of me, what I value, and my goals. My family has done everything they know how to sustain me, including loving and respecting me no matter what. For that, I thank each of them.

And Kyle: there are no words expressive enough (I've looked for them) to describe the incredible mixture of patience, consolation, encouragement, and absolute dedication that you have given to me in this pursuit. This would not have been possible without you. 


\section{ABSTRACT \\ ULTRAKINETIC FEATURES OF ANIME TEXTS: \\ REVISIONING COMPOSITION THEORY \\ AND EXPLORING VISUAL RHETORIC PEDAGOGY}

Karen O'Connell Ware

August 11, 2009

Our contemporary culture is laden with a glut of visual stimuli: advertising, packaging, television, film, the internet, digital-camera-wireless-web-access-mobile-phones. In a world filled with visually rhetorical media, it is imperative that the field of Composition continue to embrace the theorizing and use of visual rhetoric an apt (if not vital) pursuit. It seems a responsible choice to select visual texts and/or visually rich writing assignments in order to address the current fabric of public/private communication. Furthermore, such texts can potentially set the student reader (viewer/ writer/ designer/ composer) at ease in the sometimes daunting task of becoming a "college writer" and subsequently a professional able to communicate in the contemporary world. Visual texts that reflect the social climate students encounter each day may help break down some of the initial barriers to reading and composing. Students react differently to film, comics and other media than to a more traditional "school" text. This dissertation submits that the genre of Japanese animated film, known as anime, is particularly ripe for study and use in composition. Anime offers visually rhetoric texts that are accessible yet challenging for students in the area of analysis. Anime also encompasses a wide range of themes and styles. A specific form of anime, termed in this dissertation ultrakinetic, 
describes visually rhetorical texts that highlight the presentation of movement in ranges from stillness to slowed to hyper-fast. Ultrakinetic texts reflect a $21^{\text {st }}$ century sensibility and are effective models for students who must learn to read and compose in a rapidly changing multimodal environment. 
ACKNOWLEDGMENTS

\section{CHAPTER}

I. VISUAL RHETORIC IN CONTEMPORARY COMPOSITION: A SURGE OF

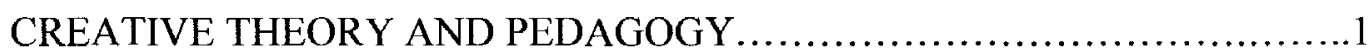

II. VISUAL RHETORIC THEORY AND PRACTICE IN COMPOSITION: A LITERATURE REVIEW .28

III. ANIME, COMPOSITION AND THE ULTRAKINETIC TEXT ...............66

IV. TOWARD AN ULTRAKINETIC VISUAL RHETORIC PEDAGOGY ........95

V. IMPLICATIONS FOR RESEARCH AND PRACTICE IN VISUAL RHETORIC ............................................. 127

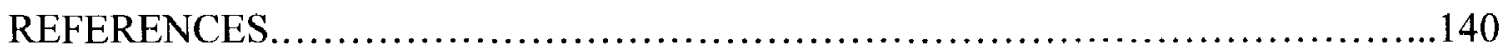

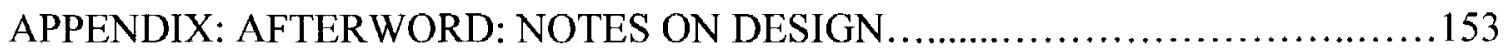

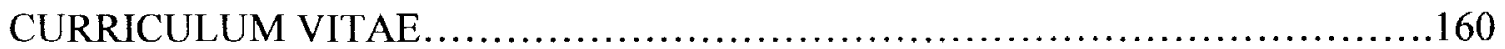




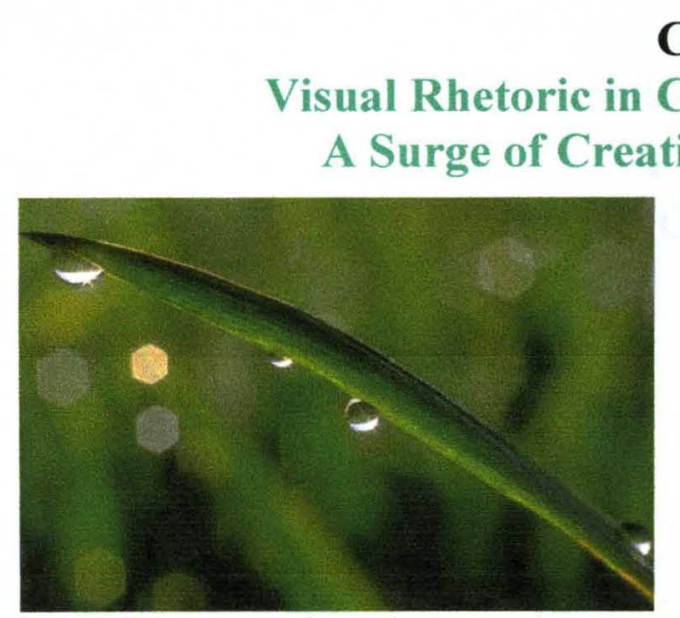

You are worried you don't write?

Don't be. It's the tribute of the air that your paintings don't just let go of you... Don't complain, my dear,

You do what I can only name.

$$
\text { --Frank O'Hara “To Larry Rivers” }
$$

The association between the visual and the verbal has been a sometimes love-hate relationship in which practitioners of one form have either admired the greener grass of the other, or suspiciously disparaged it. Artist Larry Rivers and poet Frank O'Hara are one of the first examples that I had the occasion to study how interconnected modes of expression can be. O'Hara and Rivers were a subject of study in my undergraduate work in literature, resulting in a project that would later become the impetus for my M.A. thesis. Between O'Hara and Rivers, the intellectual love affair is clear. My excitement for exploring such ideas now has a near sixteen-year history. This dissertation represents engagement at a different level; beyond synthesis or clarification, toward a contribution to the field that requires this personal perspective.

\section{Chapter 1}

\section{Contemporary Composition:} Theory and Pedagogy

In San Antonio in 2004, I attended my first 4Cs. After I stopped thinking about what I could wear that would make me appear smart, serious, and as if I belonged, I developed another worry. I found myself simultaneously searching eagerly in the program for visual rhetoric subjects of interest and equally hoping that there would be some absence-some room for me, my interests, my work: a chance to make a contribution. One of the half-day workshops was entitled "Visual Rhetoric and the Teaching of Composition." The conference program describes the impetus for the content of the workshop: "The relatively new field of visual culture studies

Dear Reader: This initial page signals my desire to p esent this argument in a visually rhetorical manner that asks you to explore the pages even as the content of the pages explore the subject matter. As is most of the information we encounter on a daily basis, some of the content is parallel, enveloped, tangential, essentially simultaneous, packed, un-packed and otherwise delineated. In many ways, the reader is the live thread that ties. 
Our contemporary culture is laden with a glut of visual stimuli: advertising, packaging, television, film, the internet, digital-camera-wireless-web-access-mobile-phones. Even our once dependably bland "serious" black and white newspapers have succumbed to multicolored image heavy pages (and may further succumb to a digital-only existence). Composition-particularly FYC - has long been a gatekeeper for the university, not just in the enculturation of matriculating students to the college chosen forms of communication, but also to critical thinking and analysis befitting postsecondary education. In a world filled with visually rhetorical media, it seems imperative that the field of Composition embrace the theorizing and use of visual rhetoric an apt (if not vital) pursuit. Composition necessarily chooses content with which to practice the process of writing. It seems a responsible choice to select visual texts and/or visually rich writing assignments in order to address the current fabric of public/private communication.

Furthermore, such texts can potentially set the student reader (viewer/ writer/ designer) at ease in the sometimes daunting task of becoming a "college writer." Critical thinking, analysis and college level communication can all be developed from the investigation of a multitude $\rightarrow$

has gained significance in the last decade as scholars examine questions of

representation and perception. While there is demonstrable interest in visual culture in composition courses, insufficient attention has been paid to the pedagogical strategies needed to develop students' skills in analyzing and creating visual arguments" (63). So I was validated in both directions regarding my concerns. The description of this workshop was evidence that this area of criticism is important, but not yet exhausted. To be sure, images and imagery continue to enter the contemporary classroom in new ways, both through intentional inclusion by instructors and peripheral invasion by current cultural elements of the visual. In "The Ecstasy of Communication," Baudrillard describes this developing environment of multiple modes: "today there is a whole pornography of information and communication" (150). The use of the term pornography indicates that not all of the expressions of these modes are making a meaningful contribution (somewhat parallel to my use of invasion). Thus, it is particularly important to investigate the issues our current students need to address. The 
$\rightarrow$ of core material. The texts selected for classroom use can be forms that are more familiar to students. Visual texts that reflect the social climate they encounter each day may help break down some of the initial barriers to reading (and writing). Provide students with a comic book or a film to read and the reaction is quite different than to a more traditional "school" text. Many so-called academic or canonical texts feel very distant from student experience. This distance places an instant hurdle between the student writer and the business at hand: thinking through writing. Modern readers, even, despite some great efforts toward inclusion of mulitcultural essays with up-to-date points of view, still have the weight, heft, feel of a textbook. So this begins to make a case for why choose a visual text. Then, what visual text should be chosen? Some interest in the field of Composition has turned to the literacy of the visual (and interactive) text of video games, James Gee's work a prime example. Quite a bit of attention has been paid to computers-and- comp (web design, online research, online communities, electronic communication, digital writing centers, etc.). Film and television have also been pop culture favorites, especially for themed courses. I propose that the genre of Japanese animated film, known as anime, is particularly ripe for study and use. Anime offers visually rhetoric texts with a certain sense of accessibility while challenging students in the area of analysis.

In the spirit of Sirc's use of word/theory equations: analysis $=$ critical thinking $=$ argument construction $=$ Good Writing (

Anime is a mode that is specific enough to offer definable conventions, yet broad enough to address a variety of areas of interest for students (and Compositionists).

SOSP

documents that students are asked to be able to read and compose have changed and continue to evolve. Our field of rhetoric and composition has been at the forefront of the university's efforts to address this issue of preparing students for their professional lives. In so-called "real life," how often are we challenged to write a quality college-level essay in comparison to the demands for being able to produce a quality PowerPoint? The focus of the 2008 Thomas R. Watson Conference directly embraced this exploration of the reading and production of different texts. The conference brought scholars together to 
"explore the complex and semiotically rich challenges we face, in the university and beyond, as we move toward new modes of composing, new forms of rhetoric, new concepts of texts and textuality and new ways of making meaning" (http://louisville.edu/conference/Watson). The 2008 Watson provides essential points of discussion regarding multimodality and the production of such texts, but the use and efficacy of visual rhetoric in the composition classroom is, as yet, an aspect of developments in composition still ripe for exploration and theorization. In Composition, there are theory-based texts and respected scholars presently working in this area (Johnson-Eiola, Selfe, Sirc, Vitanza, Wysocki, and others), but such work is not exhaustive and often is concerned with visual elements associated with technology. In Nostalgic Angels, for example, Johnson-Eilola delivers a poetic examination of the possibilities, promise, and difficulty of hypertext: "In hypertext, we are like angels without maps, suddenly gifted with wings discovering not only that we cannot find heaven, but also that walking made us less dizzy, that our new wings snag telephone wire and catch in door frames" (13). In one of her many explorations of developing technologies, Wysocki later reminds us that the relationship of word and image has been a long-term interaction, and that technologically mediated compositions like hypertext bear particular critical engagement ("Seriously Visible" 2003). Vitanza's online treatises provide excellent examples of what Johnson-Eilola calls the dizzying effects of hypertext (see "CompoZing comPLIcating Processes" in PRE/TEXT and "Abandoned to Writing: Notes Toward Several Provocations." in Enculturation, for just two such instances). Sirc's interest in action painters as models of composition and Selfe's close reading of advertising are exceptions to the frequent technological focus that Composition has had 
regarding visual rhetoric. There remains academic space for focused research regarding visual elements, their use, and questions of their effectiveness in the composition classroom. Some researchers in composition have turned to other fields like communications and art for theoretical grounds for visual rhetoric (Berger, McCloud, L. Scott, Stephens). This dissertation project represents a more conscious effort to investigate visual modes, visual rhetoric, and teaching students to write. The content focus is anime, but the larger implications of the development of theory specifically in visual rhetoric and composition will not only inform the practice of those currently using visual texts in the classroom, but also will serve as grounds for other instructors to consider the positive effects of a VISUAL RHETORICAL PEDAGOGY. I address questions of why developing theories of visual rhetoric are meaningful for composition, what current theories may inform an understanding of visual rhetoric and what visual rhetorical pedagogy may be suggested by these theories. Teaching has evolved in response to personal experiences that have included many influences. The work of members of the New London Group, such as Gunther Kress, has guided my understanding of the breadth of possibility in the study of not only a diverse range of modes, but the larger concept of multimodality. I discuss in the Literature Review in Chapter Two how particular scholarly influences have led to my desire to relay this study in a personalized context.

\section{Research Questions}

Donald Schon's work on reflective practice is integral to the development of this project. His 1983 book, The Reflective Practitioner: How Professionals Think in Action, is classified traditionally as a business-applicable sociology text, but has generated significant attention across disciplines, including composition. Though 
Schon's argument is now more than 25 years old, its core understanding is timeless: "Problems are interconnected, environments are turbulent, and the future is indeterminate" (16). His ideas on the academic vigor that non-traditional ("exploratory") experiment inherently exhibits help explain some of the work that a contemporary reflective practitioner of composition performs in the classroom when she "tests" a method of instruction. Schon guides my understanding of how to develop questions in the act of research [experiment]:

In the most generic sense, to experiment is to act in order to see what the action leads to. The most fundamental experimental question is, 'what if?' (145)

My Schonian exploratory experiment question is as simple as, "What if we use anime texts in Composition?" My research question is, "What is the impact and efficacy of using anime texts with composition students?" I hypothesize that the use of anime texts in the Composition classroom (or in the academic research of professionals in the field) provides a vocabulary for the use/exploration/discussion of visual rhetoric, multi-modality and the concept I have dubbed the ultrakinetic text. The use of anime, including ultrakinetic anime, also yields a pedagogy that guides and informs the creation of multimodal texts. Furthermore from Schon:

Exploratory experiment is essential to the sort of science that does not appear in the scientific journals, because it has been screened out of the scientists' accounts of experimental results (perhaps because it does not conform to the norms of controlled experiment). 


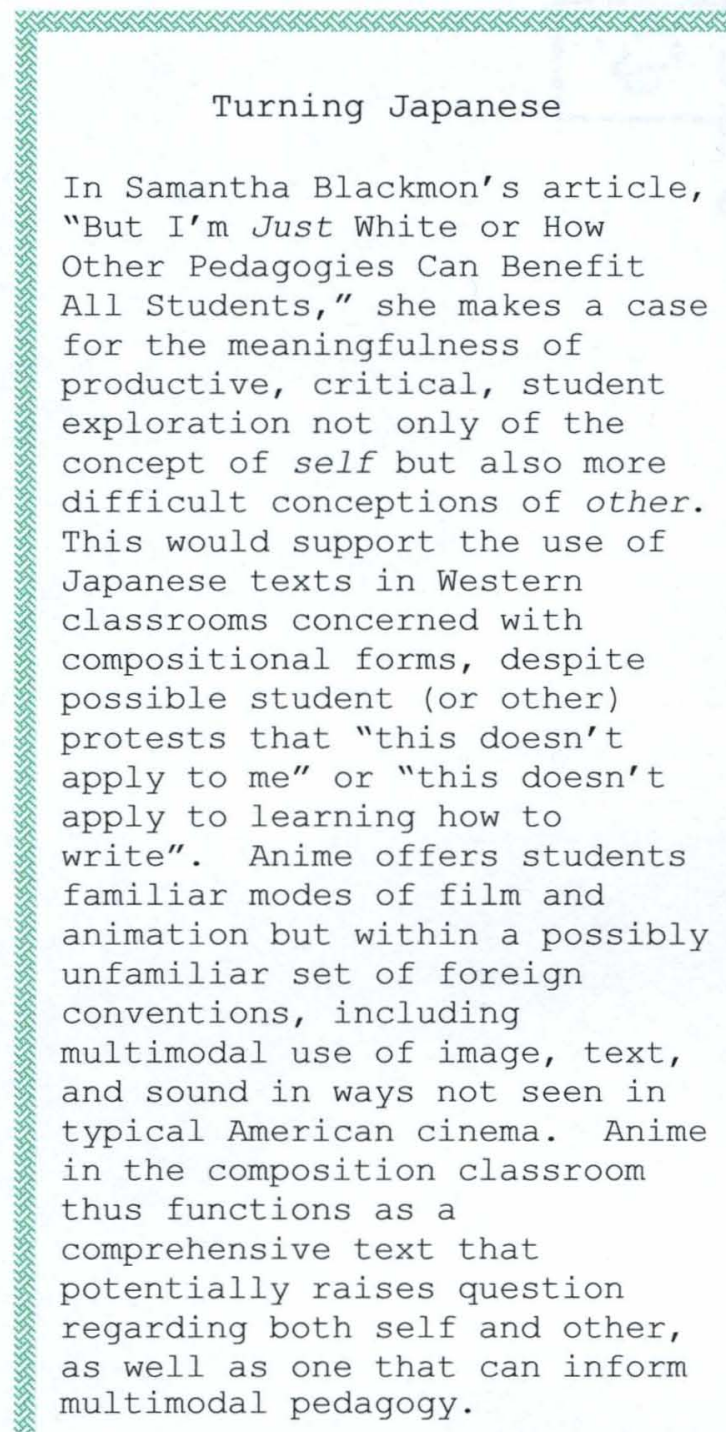

\section{Exploratory experiment is the}

probing, playful activity by which we

get a feel for things (145).

I perform Schon's "exploratory

experimentation" in the multi-modal visual

form of anime. This leads me to

supplement my exploratory experiment

and research questions with the following

supporting inquiries:

How can Composition embrace multiple

modes, including visual texts like anime, in the classroom?

What do specific anime texts have to offer

Composition?

What are the practical applications (i.e.

assignments) inherent in anime

\section{associated pedagogy?}

What are the implications of this study?

Composition as a discipline finds itself responsible not only for researching and theorizing the writing process, but also for the various materials brought into the classroom for inspiration and debate. M. A. Syverson of the Computer Writing Research Lab at the University of Texas at Austin reflects on "visual rhetoric," Composition 
pedagogy, and work on the World Wide Web from a comprehensive perspective that, for

my purposes, is worth presenting at some length:

We are guided in our teaching by pattern languages (for example, workshops on drafts, message forums, or class email lists) that support recurring patterns of activity (such as interaction and conversation between students, ongoing improvements in a piece of writing, and so on). These pattern languages are both cultural and personal; they give shape to learning environments even in new and unfamiliar media. We can learn a great deal about thinking through these media if we are willing to pay attention to our own composing processes. Visual rhetoric, too, has its pattern languages that we can discover no only through formal training, but also through our own inquiry and experimentation. In this way we can continue to expand our capacity to develop teaching and learning environments that push well beyond our original training and expertise (175).

Syverson advocates this "inquiry and experimentation" as a viable method for instructor and students alike. Schon describes how "reflection-in-action is a kind of experimenting" (132). Comic book artist and visual rhetoric theorist Scott McCloud describes his chosen mode of comics as a form of experimentation and himself as a "comics scientist" (from the March 2007 Hite Art Institute lecture at the University of Louisville).

As evidenced by Syverson's work, computers and composition is one place in composition studies where concepts in visual rhetoric are not

Out of the Mouths of Babes:

A Lesson in Visual Rhetoric (Twenty-Five Years Old!)

In Language Stories and Literacy Lessons, Harste, Woodward, and Burke present examples of child literacy events. Several instances indicate that the impulse for clarity in communication necessitate a combination of word and image-based text. Alison, age six, transcribes a shopping list for her father: "Alison wrote each of the following items as they were dictated: MOLK (milk), VONOL (vanilla), ROSBOREJAM (raspberry jam), and BOD (bread). When she was asked to write 'newspaper' she asked, 'Can I draw that?"' (164). Other examples highlight the adept conceptual meaning-making children display in response to familiar images such as corporate logos: "Nathan, age 3 was shown a carton of Crest toothpaste and asked what it said. Nathan responded, 'Brush teeth.' To Nathan, C-R-E-S-T was not an abstract symbol, but a concrete index to a particular form of social action" (143). 
only being explored, but also echo other developing theories. With web design, publishing and hypertext as key issues for those working with computers and comp, visual rhetoric has begun to take a place at the forefront of theoretical, critical and practical scholarship. Such interest has now spanned more than a decade. Mike Markel's 1998 work, "What Students See: Word Processing and the Perception of Visual Design," in Computers and Composition is the first to carry the heading of "Computers and Visual Literacy" in the table of contents and also the first to include visual rhetoric as a keyword. Computers and Composition devoted two entire issues to "Digital Rhetoric, Digital Literacy, Computers and Composition" (Vol. 18, issues 1 and 2, 2001). Enculturation, an online journal of rhetoric and writing, also produced a special issue on Visual Rhetoric (Volume 3.2).

Computers are obviously not the only mode of visual information; other sources may be investigated as well, including first year composition classroom darlings like film, television, and other image-rich media. Multiple classroom uses may be further explored, especially in light of the direct consequences that theory, and the practices thereof, have on individual students. Theoretical work in visual rhetoric will offer portable and sustainable techniques derived from the experience of individual instructors and extrapolated to successful methods for writing practitioners as a whole.

Geoffrey Sirc's English Composition as a Happening connects the composing that occurred in the creation of art in America in the 1960s and the process of composition in writing. Other theorists have also explored how composing a text is a visually oriented pursuit (Arnheim, Berger, Bernhardt, Kress, Wysocki). What is unusual about Sirc's description is that he uses a period in art history to explore the history (and future) of 
composition. At the core of this journey is the idea of returning to the meaning of the word composition itself. In doing so, Sirc creates a world of theory and practice. Composing processes in art, in media, in advertising, in business writing, in first-year composition have developed in various ways and have much to learn from one another. The common denominator is composition: the construction, organization, even decoration, of the chosen materials to accomplish a desired rhetorical effect.

According to Sirc, the problem with current composition theory and practice is that we have spent too much time erecting boundaries for composing when such lines should be blurred to match the reality of "real" composition work in our daily lives. Composition courses have been co- opted for purposes other than the exploration of composing Sirc criticizes the idea of composition bearing some responsibility to other departments to "get students ready" for coursework in other disciplines. He also finds fault with the turn to cultural studies, indicating such thematic material evokes a political agenda in the guise of the discovery of personal identity. These points are arguable, but his idea is that composition needs not only to concentrate on the business of composition, but it needs to find ways to get excited about it.

Sirc's history depicts composition as following the aesthetic of modernism in its search for ways to perfect final products. Even writers who couch their arguments as revolutionary (Bartholomae is central to Sirc's criticism here) are reiterating a certain kind of product-oriented composition. Sirc wants composition's more radical potential to be examined, for the composing to be idea-generative rather than product-oriented. 
Sirc's desire to see the "disenchantment" in composition theory and pedagogy lifted through a less modernist, more "happenings" approach parallels my argument for the efficacy of the inclusion of visual modes like anime into the writing classroom. Though Sirc is on a path of his own, mine steps by his side directly in enthusiasm for creative, contemporary pedagogy that values process and is actively reflective regarding methods and materials. Sirc's happening makes room for my ultrakinetic anime texts.

For Sirc, the valorization of idea-generation or products that are continually in process renders the concept of the "imperfect" of particular importance. Dadaist artist Marcel Duchamp is one of Sirc's key examples in this area. Duchamp's compositional practice of using common objects ("readymade") with little or no alteration is a great example of allowing the imperfect to speak; somewhat as process theory values each draft of a written composition. Sirc presents an email from his student, Greg White, which may formally be read as errorridden, but in content is thoughtful, industrious and persuasive: a form of "readymade." It is important to Sirc to recognize the artist's process (regardless of whether that artist is Duchamp or Greg White).

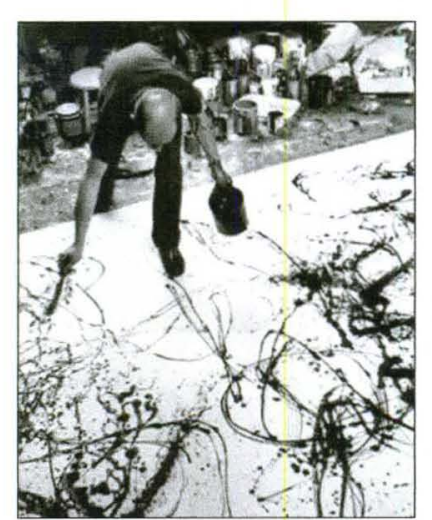

Sirc highlights Jackson Pollock's work as the "King of Process" as particularly productive for writing compositionists to consider. The drip paintings communicate a story about how the paint arrived at its location on the canvas, a story 
BIG C COMPOSITION is the field we know often as Rhetoric and Composition; little $\mathrm{c}$ composition is about the creative combination of information to imply meaning.

BIG C COMPOSITION is not a primary concern for everyone. Little composition, on the other hand, embodies elements of what it means to be a human being who tries to communicate: the construction of a readable "text".

about the process, movement,

emotion and action of painting

"Little c composition" as discussed here is heavily influenced by Zebroski via Huot. In a Teaching of Writing course (Eng 602) at the University of Louisville,

rather than a presentation of

static imagery. Perhaps if, as

Huot presented the terms "big T Theory" and "little t theory" as a vocabulary for discussion of the ways in which our theories of language affect our practices as instructors.

Sirc might suggest, we move

to viewing our pages and

screens as canvases on which

we create with materials, the

connections between

composition (creating "art”)

and composition (writing) will

be more apparent. It is in this

spirit that I have selected the

multimodal form of anime as

a focus point for my own

discussion.

My dissertation research

centers on more clearly

Big T Theory = abstract articulation of principles; a "grand statement" [traditional theory]

Little t theory $=$ Zebroski's concept of pedagogical actions being theoretical, whether or not the practitioner is conscious of her theories and assumptions. [theory-inpractice]

For example:

Practice:

Little t theory:

Use of peer groups

Writing is a social activity; writers profit from interaction

Marking all student error on drafts

Students learn to correct errors by being corrected; Correctness is important in all drafts

(Big $T$, little $t$ explanation and example from classroom materials by Huot.)

In the Introduction to Teaching Writing with Computers, Takayoshi and Huot describe this concept succinctly:

Zebroski's point is that any teaching practice we might consider comes with a set of beliefs and assumptions. For example, if we have students work on exercises of grammar, usage, and mechanics outside of their writing, we're assuming that language use can be learned outside of a communicative context. Or, if we break our classes into groups so that students can respond to each other's writing, then we're assuming that effective writing and its learning require a social dimension within which students need to read, write, and respond to each other. Failure to examine our beliefs and assumptions about teaching can lead to what George Hillocks calls teaching as a 'protected activity' (4-5). 
delineating the connection between composition and contemporary visual forms (the selected form of anime will be used to narrow the illustration) in order to help theorize visual rhetoric in composition. This "exploratory experiment" in visual rhetoric represents particular reflection on my own teaching practice. Influenced by work regarding multimodality, its importance in our culture, and its effect on study and teaching, I suggest that visual texts like anime provide not only content focus for composition, but also present implications for why instructors should consider the positive effects of a visual rhetoric pedagogy that exhibits awareness of multiple modes. My study offers the concept of the "ultrakinetic text" as particularly timely for consideration in a composition research climate that has embraced popular culture, multimedia, and multiple literacies.

\section{Ultrakinetic}

A developing definition: "Ultrakinetic" visual texts focus on the importance and visual

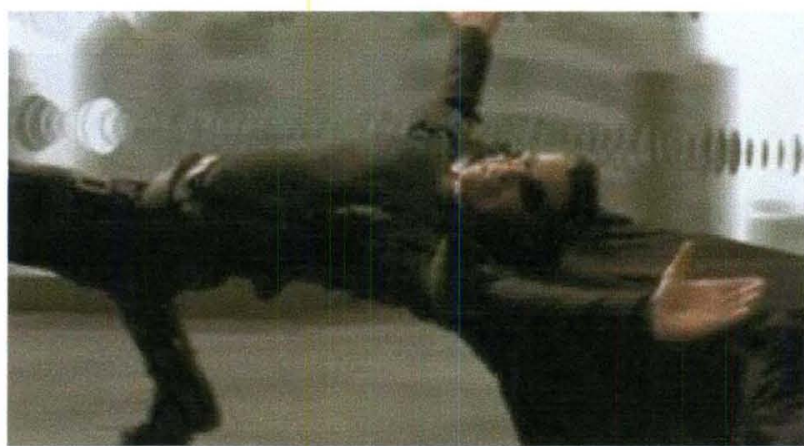

cuts that would be more appropriately termed

Bullet-time scene from The Matrix and for multiple reasons. The meaning of the prefix "ultra" helps designate the significance of movement in certain texts without relegating it only to the pleasure of movement in various ways sort of rapid-fire MTV generation quick hyperkinetic. The "bullet time" technology used in

The Matrix films is an example of how slowing down the action can emphasize the movement in ways real-time filmmaking techniques do not. The term ultrakinetic owes a conceptual debt to both the novel and the film version of A Clockwork Orange where ultraviolence refers not just to horrific physical assaults, but also the psychological terror of such acts as home invasion, the destruction of property and, finally, the ultra-violent act of brainwashing. One violence may be conceived as rapid or hyper, the other, a slow 
motion version. These distinctions drive the reader of visual texts to understand the ways

in which motion is a temporal issue, particularly in our contemporary technology-laden

environment. It is a timing different from what we experience in reality: it highlights and

heightens the subject matter presented through the manner of presentation itself. Anime

provides a multitude of articulations of what I am calling "ultrakinetic" text. These will

be explored in Chapter 3:

Anime, Composition,

and the Ultrakinetic

Text.

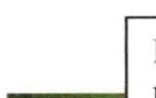

Kurosawa's Dreams is an excellent example of the emphasis of motion in its many forms. In this scene, the attention of the viewer is fragmented by the presentation of a number of richly decorated characters for consideration, while the actual movement of the figures is painstakingly slow, the volume of motion allows the reader little clue of where to look. contemporary composing process.

The use of popular culture texts, including those that may be termed ultrakinetic, in the first-year classroom may be criticized for providing students with the same content they receive in their private pursuits, rather than enculturating them into the academic community. Critical analysis, however, is a skill that may be practiced in the examination of any text. It seems intuitive to select texts that are rich with fuel for discussion, yet somehow accessible to the first-year student. Popular culture media choices have this effect. After watching and discussing the mainstream film Fight Club, a student in a 101 section I taught remarked how difficult the task proved to be even though we were "just watching a movie and talking about it." I replied that was only one way to describe what our task had been: "You could say you were performing a critical analysis of a visual text." This response seemed to satisfy the student that the work she was doing was indeed "easy" only in that it seemed strange to watch a movie as a part of a "serious college assignment."

First-year composition students are not limited in ability by the sensory overload of images and other modes of communication encountered in contemporary daily life. Time spent updating Facebook and sending text messages has not dulls their ability to critically read texts like Fight Club or even an essay by Friere. Frequently, applying known methods of reading and deconstruction to an approachable familiar text (i.e. "watching a movie and talking about it.") guides the student process of adapting to a more unfamiliar or seemingly unapproachable text (like Friere). The inclusion of visual texts in the classroom addresses the immediate concerns that a visually laden environment incites, it also provides opportunities for students to consider producing visual (or other modes of) texts themselves. Scott DeWitt, a compositionist who writes mostly in the area of technology and the writing classroom, posits that we must instruct students to be responsible and critical readers of visual media. Consistent practice of this critical analysis leads to the development of core methods of argumentation. Enter the mediasavvy student critic, capable of applying that skill to reading and producing texts in multiple modes. 
My research includes a variety of scholarship that allows me to explore current work in composition addressing the visual, outside disciplines and theories that may help inform the issue, and examples of visually rhetorical texts and their criticism. Also included are texts that help substantiate and define the concept of the "ultrakinetic text" as well as texts that have played a role in my own reflective practice.

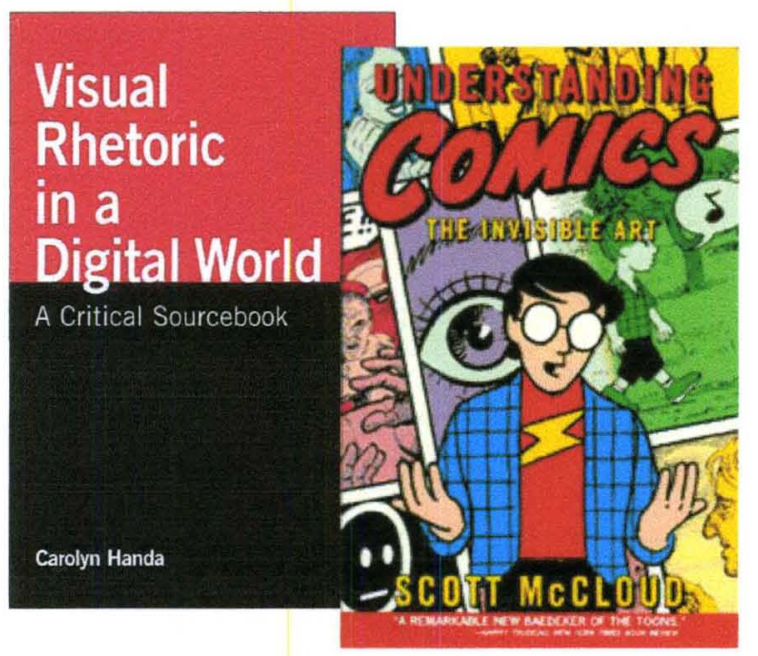

Carolyn Handa's collection, Visual

Rhetoric in a Digital World: A Critical

Sourcebook, presents a "critical

sourcebook" selection of scholarship

regarding visual rhetoric and technology.

Like many texts, however, the arguments

focus on visual rhetoric without

significantly utilizing visual rhetoric. Handa explains in her introduction the impetus for what she terms "Placing the Visual in the Writing Classroom": "Composition teachers are thinking about the visual, considering theories historicizing the separation of words and images, and understanding the place of classical rhetoric, design studies, and cultural studies in our pedagogy. However, there is still much work to be done" (2). Handa acknowledges the breadth of possibility, but largely does not present materials reflective of their content in their design. The chapter from Scott McCloud's Reinventing Comics (2000) is a noted exception. McCloud's argument walks its talk: theorizing the importance and impact of the visual within a visual format. In 1993, McCloud broke new ground with Understanding Comics, his original foray into presenting theoretical concepts within a visual style. He draws (no pun intended) on his strength and 
knowledge base as an artist to help him validate his argument, using a familiar form as well. The familiar form to most scholars is not the sequential art of a comic book.

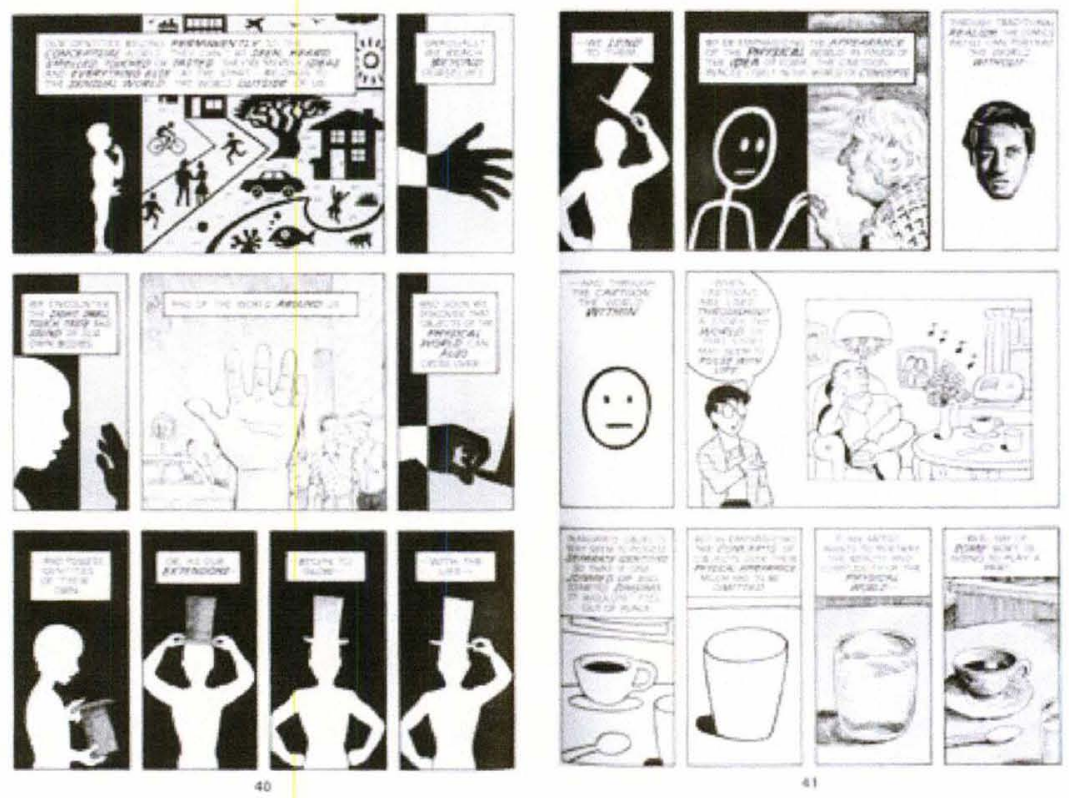

Rhetoric and

Composition scholars

are quite used to

reading and writing in

traditional, linear

textual forms with little

or no intrusion by

images. We have all

written articles and

theses for which preferred fonts were designated, margins were measured and images

were carefully labeled according to MLA or APA regulations. But if McCloud's 1993

text yielded such a positive response, why does Handa's collection more than a decade

later look so drab, so disconnected from its subject matter? Furthermore, why does

Handa not address how different McCloud's writing is in her discussion?

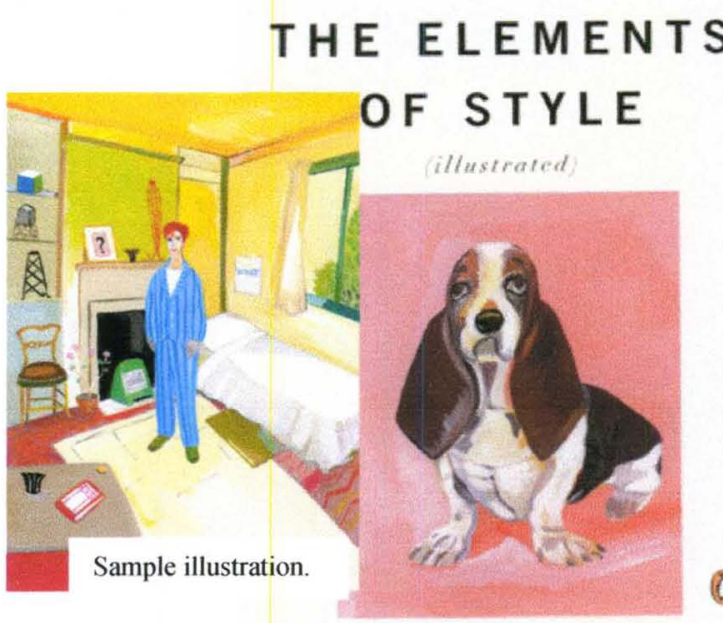

\section{Sign $O^{\prime}$ the Times}

In 2005, Penguin Books presented a new edition of the inescapable classic, Strunk and White's The Elements of Style: illustrated by Maira Kalman, an artist frequently featured in The New Yorker. The publisher describes the attraction of adding the images: "The only style manual to ever appear on bestseller lists has explained to millions of readers the basic principles of plain English, and Kalman's fifty-seven exquisite illustrations give the revered work a jolt of new energy, making the learning experience more colorful and clear." 
In The Rise of the Image The Fall of the Word, Mitchell Stephens recognizes that despite his argument of the movement of communication to the ubiquitous visual form of video, there remains a certain valorization of the printed word. His argument, for instance, is presented in $a$ book, not a CD ROM or web hypertext or even a DVD or video. The intimation is that the printed word will retain value in our culture no matter how proliferated technologies become. In reading, discussing, and producing multimodal texts the participants in the composition classroom are not inciting a revolution against the traditional essay, they are joining a community that seeks to communicate in the best possible mode(s) available. In Literacy in the New Media Age, Gunther Kress does not argue against the importance of "the word," rather he depicts an evolution in communication as "the change in media, largely from book and page to screen" (5). Furthermore, he explains that what Stephens terms the "rise" of the image is essentially the layering of image-based reasoning: "writing will more and more become organized and shaped by the logic of the image-space of the screen. This is one inescapable effect of the potentials of the screen, and the technology of the new media" (20).

\section{Anime: A Multimodal Visual Feast}

In addition to providing excellent examples of the ultrakinetic text concept, anime is a particularly useful visual resource for inclusion in composition pedagogy due to the richness of the medium, its layered modalities, and breadth of content and contexts. Anime is also a source that is certainly and obviously connected to screens.

It is important to address a few of the possible concerns and assumptions different audiences may have regarding anime. Hayao Miyazaki, director of popular anime films 
such as My Neighbor Totoro, Kiki's Delivery Service, Princess Mononoke and Spirited Away, has indicated that he prefers his work to be referred to as "manga films" rather than anime due, in part, to the frequently ignorant responses people normally have to the term. Some audiences assume that anime means another version of cartoons for kids. Attempts to disabuse this label by describing some anime as "adult" has presented an equally inaccurate impression that anime is predominantly pornographic in nature. Anime is less a genre and more a medium for expression with inherent conventions and available techniques similar to other media forms.

One of the techniques or conventions found in anime that will be key to the discussion of the ultrakinetic text is the combined visual/verbal modality that helps articulate moments in time. For example, in the generally violent story of the film Kill Bill, Quentin Tarantino turns to anime when the narrative becomes too intense for live action presentation in the back story of Oren Ishii. As a child, she witnesses the savage murder of her father by Asian gangsters from a hiding place beneath a bed. In anime mode, Tarantino is able to direct an amazingly expressive moment: as the child gasps at the sight before her, the sound of the word "whimper" is accompanied by a visual of a fragile-looking text of the word "whimper" sliding from her open mouth. Many anime works include such "written text" as an idiomatic means of punctuating moments in a given story. Word and image combine in a new visual/verbal hybrid vocabulary that is able to evoke recognition and response that word or image alone may not effect. The moment is also exemplary of the heightened sense of time and space found in ultrakinetic texts. 
The Kill Bill anime sequence of young Oren Ishii trying to capture and force back into her mouth the "whimper" that has escaped. This action would be neither necessary nor possible within the live action portion of the film. In this case, anime conventions allow for a visual moment of particular emotional impact. Tarantino is thus able to quickly and effectively portray an interior struggle for the character Oren. With this portion of the film representing her background story, it is important to have such an evocative glimpse at a fragile moment for a figure who is otherwise described in her adulthood as undaunted and vicious. In print, the moment would simply be described and Oren's interior thoughts and emotions could be detailed, but perhaps less viscerally. In the anime mode, the presentation is incredibly effective.

(Note: The screen stills presented here are moments apart in the film, not frame-by-frame.)

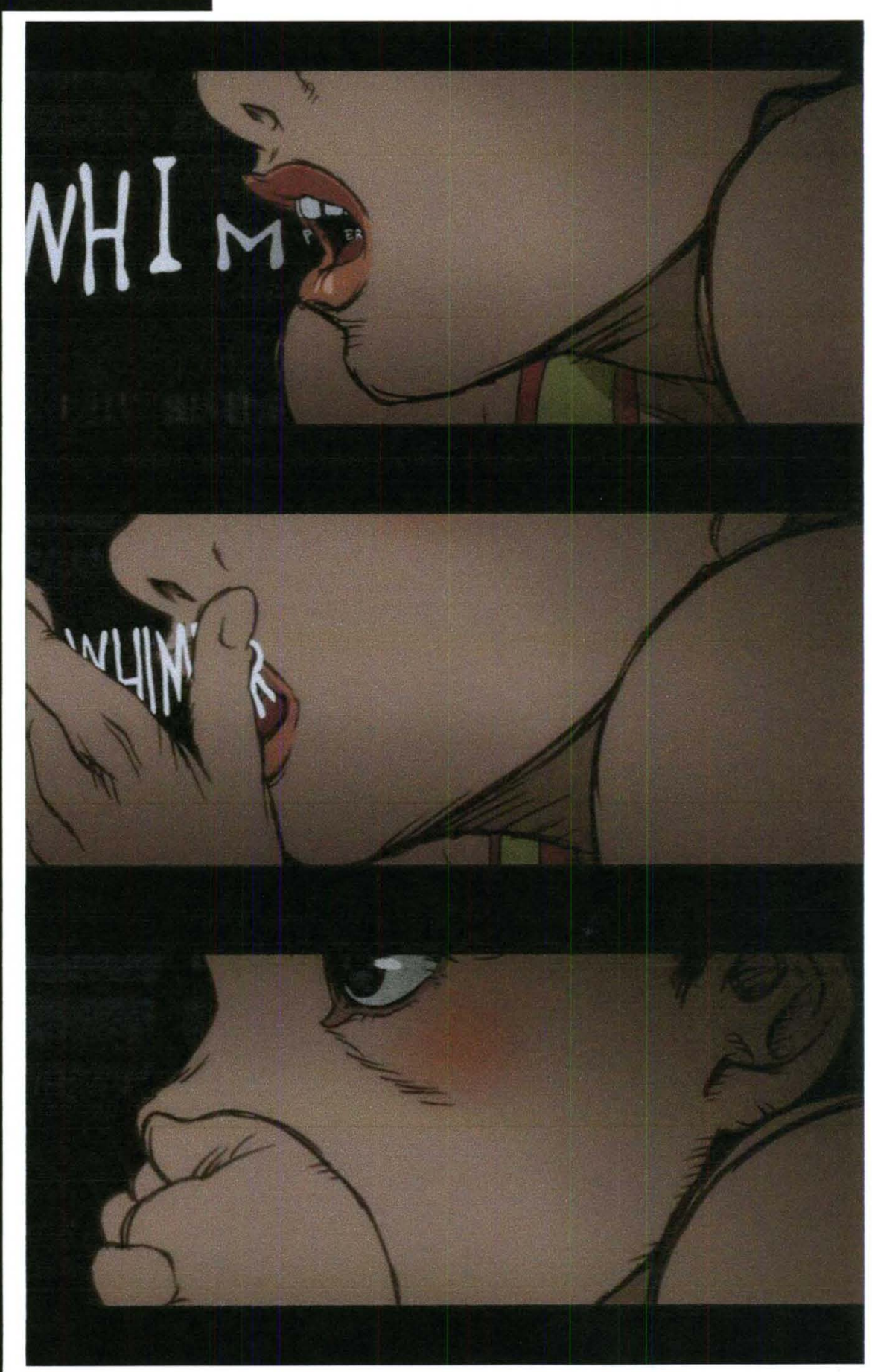




\section{A Note on Format and Contributing to the Field}

In order to address the issue in composition of a possible disparity between what we talk and how we talk about it, one of the goals for this project is to practice visual rhetoric even while examining it. The method of practice, as well, may help address some potential criticism. Laura Micciche has used the term "disappointment" to express the condition of the field of composition and specifically, the sentiment with which writing program administrators approach their work (Sirc also uses "disappointment" and

"disenchantment" to

express his

dissatisfaction with

Composition's general

climate). In Micciche's

argument, composition

instructors have all too

often been relegated to

grunt-work caretaking

in which they are

expected to "fix" what

is "wrong" with student

texts. This is an over-

simplification of what

composition is

meant/able to do. So

what is the "real" focus

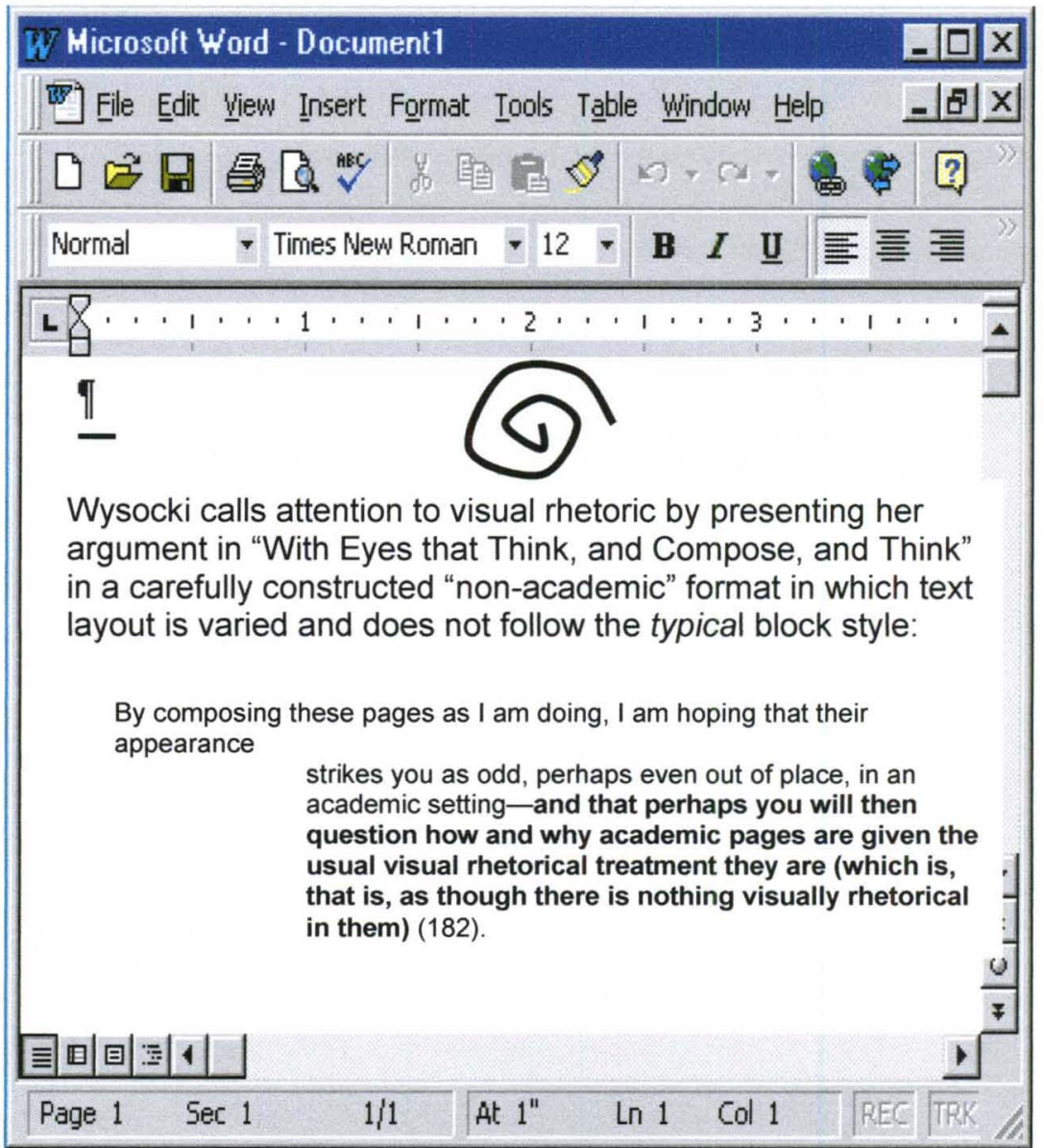

of writing instruction? Ask five people and you'll get five answers, but most would agree, I think, that grunt should not be anywhere near the job description. Writing instruction is meant, among other things, to spend a significant amount of time guiding students through the development of their own process(es) and technique(s) of composing and allowing them to practice possibilities and receive individual feedback. I argue that we need to theorize visual rhetoric because of how often visual forms enter our 
writing classrooms. The greater truth is that writing is visual. To work in visual rhetoric is to work in composition.

Integration of visual rhetoric practice may meet resistance. The protests seem reasonable: "But I don't have knowledge or experience in design...I don't have time to cover this...I'm not interested in the visual..." However, if we first recognize the ways in which we already understand writing to be visual, a foundation of knowledge is established. The act alone of functioning in a contemporary society awash with visual information a certain level of experience, one that can Texts are visually rhetorical "word-only." My

of
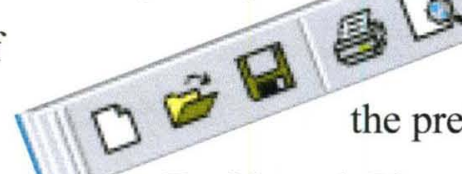
wy examination of the theorization and practice even if they may be identified as To this end, I have selected Microsoft Word, arguably the most commonly used word processing software (certainly a common inhabitant of the university campus), as the sole means for creating a visually rhetorical dissertation. The challenge is to push the envelope by working within a somewhat traditional format in a non-traditional way. In doing so, the argument for use of the visual is highlighted by use of the visual. Furthermore, the use of a program of marked familiarity for most members of my audience counters the debate that working with/producing visually

Composition Artist: A Formatting Anecdote

In 2001, I submitted to the University of Louisville a M.A. thesis with a visually rhetorical component of an additional cover title page that was meant to be evocative of the overall thesis and set a tone for the reader. Upon review by the Graduate School, I was instructed that the image would be appropriate only in an appendix, not as a frontispiece (despite the inclusion of the required "typical" title page.

Fast forward eight years later, the only caveat given by the Graduate School for the production of this image-laden dissertation has been to "try to keep your images and text boxes as in line with the required margins as possible." I was also told that "we're seeing this [pointing to a text box labeled image] a lot more in the artistic disciplines."

rhetorical documents is a foreign, technologically fraught and frequently expensive enterprise. Look, ma, if I can do this in Word, imagine what can be accomplished with software or materials specifically focused on image production. :-) 


\section{Focus/Method}

This project will also use Writing New Media (Wysocki, Johnson-Eilola, Selfe, Sirc) as a model - the juxtaposition of argument/theoretical discussion with directly related classroom assignments and activities will help me make the theory/practice connection while exploring the anime texts. Theories presented by the authors of Writing New Media have figured notably in the evolution of my own thoughts as a writer and educator. The significance of the core principles of reflective practice and our pedagogical responsibility are well-expressed by these four writer/teacher/composers: "Our conversations - and this book [Writing New Media], then — are about how we can understand these circumstances not as passive observers but as active, reflective, responsible composers" (vii). The circumstances referenced are essentially the ideas of writing being different than it has been. The product of the "conversations" is not only presentation of theory, but also real world classroom activities and assignments.

The work of noted anime authority Fred Patten provides guidance for which anime texts to select. Patten's critical work on anime is comprehensive, and he is often cited as the foremost expert on anime history and themes. My goal in the exploring a very brief list of selections is to illustrate the aforementioned focus-yet-breadth nature of anime through a variety of examples that are important in the overall anime canon. Shonen-ai ("boy love") series like Loveless, for example, helps display the variety of themes available. Akira is always ranked at the top of best-of-anime (and some best film/ best animation lists as well), including Patten's “Thirteen Notable Anime Films, 1985-99" (125).

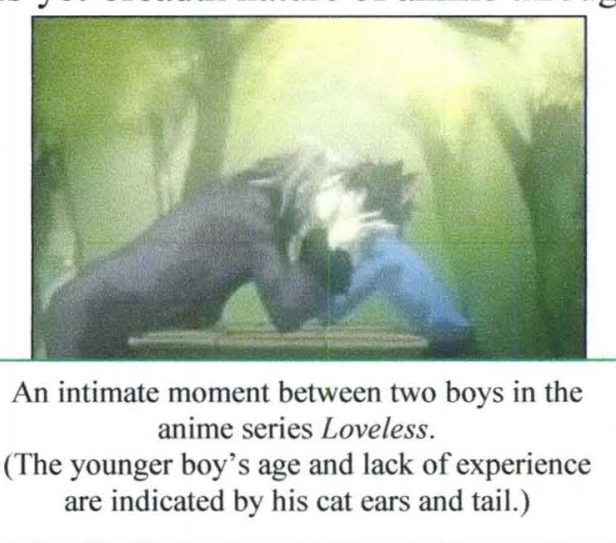

\section{Design}

\section{Chapter 1: Visual Rhetoric in Contemporary Composition:}

\section{A Surge of Creative Theory and Pedagogy}


In this chapter, I have sought to set the significance of this study of anime and the ultrakinetic text concept as they apply to Composition and in terms of the current level of interest in visually rhetorical methods. My Schonian exploratory experiment question is "what if we use anime texts in Composition?" My research question is "what is the impact and efficacy of using anime texts with composition students?"

\section{Chapter 2: Visual Rhetoric Theory and Practice in Composition:}

\section{A Literature Review}

The Literature Review will examine a selection of theory, criticism, and expressions of different modes, methods, and practices from a variety of resources, including Sirc, Berger, Selfe, and Wysocki. Arnheim's work in visual theory from an art perspective will be key, as will the writing of Garoian and Gaudelius on art and visual culture. Patten's close examination of anime, manga, and other imported Japanese modes will be joined by Susan Napier's criticism of popular anime as well as Frey and Fisher's exploration of the applicability of modes like anime in the classroom. The intricate definition and discussion of multi-modality by Gunther Kress and the New London Group provides a theoretical anchor. Pat Carini is an elegant example of the importance of personal voice and passion in academic writing.

\section{Chapter 3: Anime, Composition and the Ultrakinetic Text}

This chapter will examine the significance of and explore a few examples of what I define as "ultrakinetic" texts. Anime is a mode that provides multiple illustrations of the ultrakinetic concept. For composition study, the ultrakinetic anime text provides familiar (yet unfamiliar) material for analysis that highlights the complexities of multimodality and hybridity. I have narrowed my anime choice to five selections, each representing a different area of importance for both anime and composition: 
- Akira: This ground-breaking work is practically canonical for anime. The sample composition discussion will examine the influence of the film on hip hop/rap musical artist Kanye West's video for "Stronger."

- Loveless: This television series provides a very recent production of anime: U.S. release in 2007. The series is also an example of breadth of themes available in anime.

- Tezuka's Metropolis: Though writer Osamu Tezuka insists that he only "saw a poster" of the 1927 Fritz Lang black and white classic, this anime masterpiece is clearly a close parallel in theme, content, and character development. An instant success in 2001 in Japan, Osamu Tezuka's Metropolis was widely released and lauded by critics in the U.S. in 2002.

- Cowboy Bebop: Initially an incredibly popular television series in 1998 in Japan, it is the first anime in Cartoon Network's Adult Swim block of latenight programming in the U.S. in 2001. Sirc's interest in "happenings" music and composition will be featured.

- Grave of the Fireflies: This amazing chronicle of a boy and his younger sister in Japan after the dropping of the atomic bombs took some time to reach the U.S., perhaps in part due to its unflinching expression of the damage caused to citizens by the war. This anime provides an outstanding resource for examining context, history, personal narrative, and voice.

\section{Chapter 4:}

\section{Applications Toward an Ultrakinetic Visual Rhetoric Pedagogy}

In their recent article for WPA: Writing Program Administration, Takayoshi and Huot discuss the importance of student ability to produce multimodal texts within the context of describing a shift in thinking and focus (as well as theory and practice) for their university: "While there was some concern among certain humanities faculty that courses including multi-modal forms of composition would short-change a traditional focus on 
the print-linguistic scholarly essay, faculty across the curriculum agreed that students learning to write for the academy and a variety of professions need experience and instruction with composing in multiple, digital media" (90). In order to support the university's efforts, a proposal including a student video essay was presented to representatives of a computer company. Regarding the student essay, one representative said, "I have a staff of 30 people under me - and I wish all of them knew how to make such a composition" (92). This underscores the need for students to know how to read and produce texts in a variety of modes.

In Writing New Media, Wysocki, Johnson-Eilola, Selfe, and Sirc address this issue directly by each providing "activities" to accompany their theoretical discussions of new media in the classroom. These activities not only represent practices in multi-modal composition, they make transparent processes of the necessary conjunction of theory and practice. As Schon describes, "both ordinary people and professional practitioners often think about what they are doing, sometimes even while doing it" (50). Here, theory and practice are termed simply as "think" and "do," respectively. Writing New Media is an amazing and inspirational example of the inductive reasoning of a reflective practitioner. Following these examples of how relationships between theory and practice may be illuminated, Chapter 4 discuss a Berger-inspired assignment I call "The Anti-Essay," Molly Bang's exercise of "Arranging Shapes on a Rectangle," and Sirc's "Basic Box." 


\section{Chapter 5: Implications for Research and Practice in Visual Rhetoric}

When I first began the process of studying for the doctoral exams and preparing to propose what my dissertation would be, I was overwhelmed by the idea of "contributing to the field." This led to writing that was extraordinarily contentious, as I blasted the work of others for not being so-called aware enough, precise enough, theoretical enough. It took some time for me to discover that I wasn't angry about a lack in the field; I was excited at the possibility of joining a particular idea space. In my conclusion, I bring the preceding chapters together and look at the implications signaled by this reflective time spent with the visual rhetoric of anime and Composition.

\section{Afterword: Reflection on Design}

Since part of this effort is to experience what it may feel like to "walk the talk" and use some visually rhetorical methods while discussing visual rhetoric, some transparency of discussion in the design is warranted. For example, centering the text of the title above is meant to subconsciously signal to my reader that my descriptions have been carefully considered; the "centering" is like visual word yoga, presenting the content as weighed and balanced, therefore trustworthy and thoughtful. The green

The time involved in this multimodal process of composing is a factor I originally underestimated, particularly with my selected technology. Reaching the desired placement of images and other elements is sometimes a multiple hour pursuit to accomplish movement measured in fractions of inches.

text is meant to be a soothing shade, also an echo of not only greens used elsewhere in the text, but also my consistent use of green ink in commenting on student texts (reading notes in the traditional red ink is a sometimes jarring experience, where green ink supposedly lowers the heart rate and renders the reader receptive). 


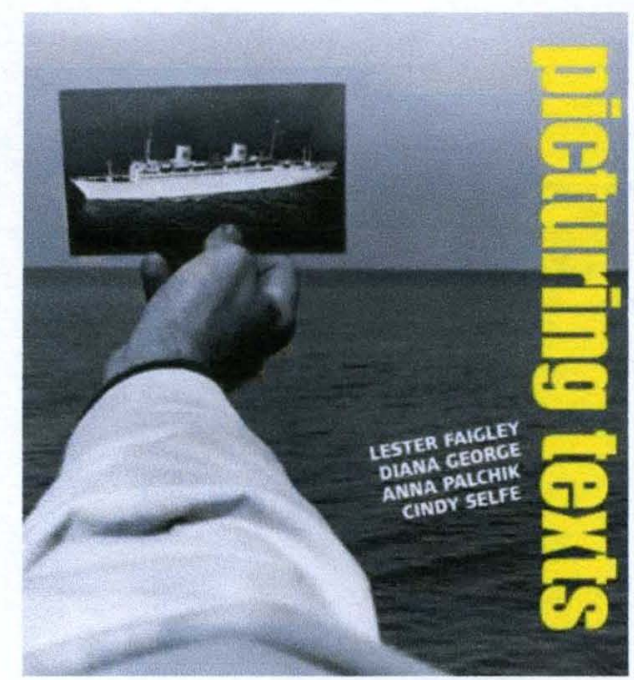

\section{Toward the (a) Beginning}

The burgeoning portion of the composition field that is exploring new media and multiliteracy concepts is filled with exciting work and dynamic scholars. I look forward to joining the discussion through the execution of this dissertation project.

With classroom material like Picturing Texts and resources like Writing New Media available, it is an amazing time for study in the area of visual rhetoric.

Time

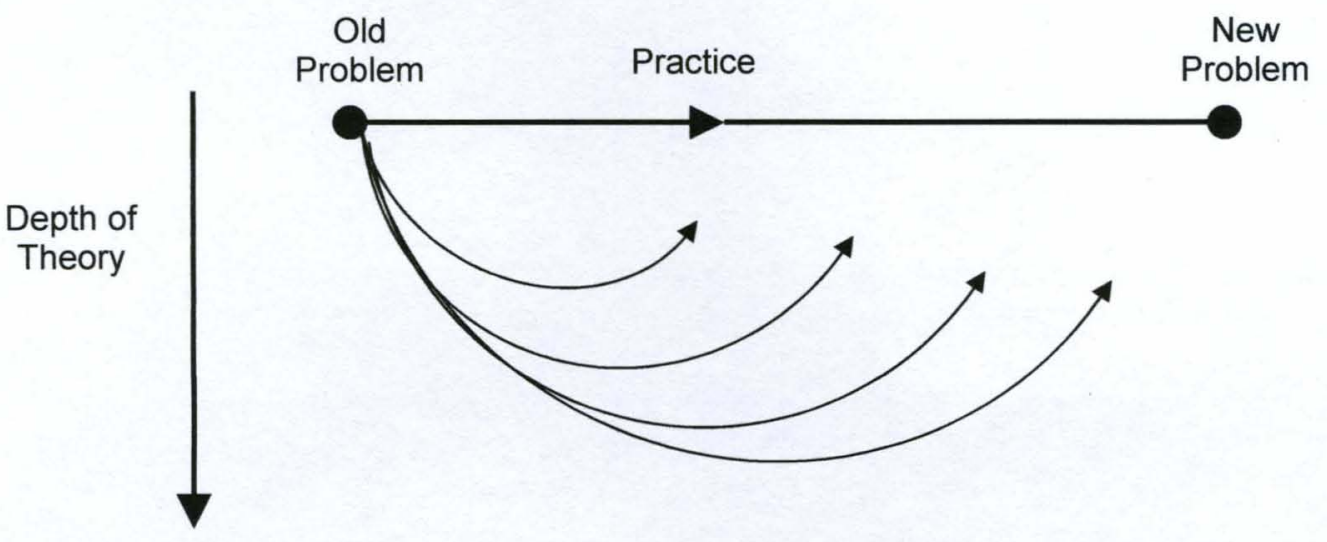

"the 'arc' from practice to theory and back (the PTP arc, for short)" (Phelps $37)$.

Over time, the reflective practioner's journey (my journey) from problem to problem, interest to interest, idea to idea, developing depths of theory. Phelps, like Schon, provides definition for the reflective practice anchor of this study. 


\section{Chapter 2}

\section{Visual Rhetoric Theory and Practice in Composition:}

\section{A Literature Review}

\section{I will write upon the pages of history}

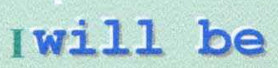

what I want them to say.

I will speak my own

Prior to my comprehensive exams, my mother bought a pen and pencil set for me, housed in a lovely fabric covered box. The lid was etched with this quote from Maya Angelou. An appropriate epigraph for this discussion of those who have already written.
To address my research question of "what is the impact and efficacy of using anime texts with composition students," I have researched not only in the field of Composition, but also in art theory, film criticism (including animation), comic book analysis, and new media studies. From this reading, I have chosen to focus on a few key texts that directly inform the study of visual rhetoric and assist me in defining not only the ultrakinetic anime text, but also its importance in the teaching of composition. For an example of effective description through personal voice and storytelling, I turn to Carini. Aristotle grounds my overall reading approach, providing an essential

characterization of rhetoric itself. Art theory and criticism is an obvious and crucial component in understanding contemporary work in visual rhetoric (Arnheim, Barthes, Berger, McCloud). The field of Communications has addressed word and image in combination and is revelatory regarding visual rhetoric (Stephens). In Composition, Sirc

In this chapter, one of the design elements presents short blocks of quotation from the resources parallel with a similarly brief response/connection/contextualization. This design element is meant to mimic the internal process of reading response; the mind instantly creates associations. On the page, this is represented by these side-by-sides. 
is instrumental in my understanding of how the field can be re-visioned, while other work provides a look specifically at visioning visual rhetoric in composition (Ramey).

Reflective practice is a necessary element in my ability to develop the ultrakinetic concept and to ask "exploratory" questions, for which Schon is the bible. No study of hybrid texts (as ultrakinetic anime are) would be complete without reference to Kress and the work of the New London Group. Finally, I examine an important critical work in anime that helps synthesize and focus these ideas on art, composition, and rhetoric within the conventions of Japanese animation styles (S. Brown).

\section{Finding a Voice}

The dissertation project—depending on one's point of view—a culmination, a summary exploration of time spent reading, researching, and practicing in a particular field or the bridge from one's "student" work to "professional" work. During my graduate studies, I would sometimes wander down the aisles of the library devoted to completed and approved theses and dissertations. What a stamp of success to be hard-bound and filed! But what differences are held in those volumes: some slim and succinct, others fat, expansive, with multiple addendums, appendices burgeoning with examples, swelling to two or more volumes. I was particularly fascinated by some of the M.F.A. theses in Art and Acting; their pages unfolding into intensely personal narratives that expounded not only on the academic process of the enterprise, but also on the emotions, connections, and individual circumstances that affected the choices the artist was making. I wished that I could infuse my work with such a voice—informed, analytical, supported and researched yet so obviously the product of a real person. 
Critical narratives infused with a personal voice have been the most effective for me as an audience. I remember reading Pat Carini's "Dear Sister Bess..." in the McDonald's near campus before class one evening. The voice, the emotion were so intense, so palpable to me, I began to cry right in the middle of the fast food restaurant. During open discussion in class that night, I shared my story and was refreshed by the welcoming reaction to my confession. The class talked at some length about the possibility of a professional discourse that could be considered both academically rigorous and still have the kind of impact that would make a graduate student cry in McDonald's.

Carini also helps me understand efficacy in pedagogical methods in terms of the core beliefs and assumptions each

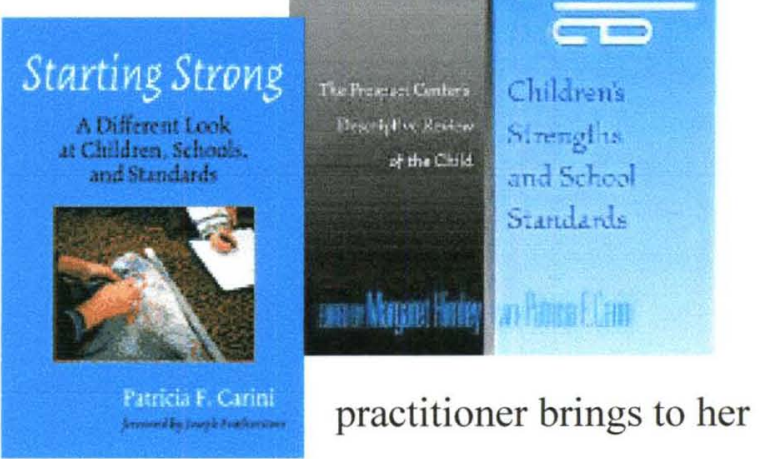
practice. For Carini, one core assumption involves what she describes as "humanness": I offer humanness as widely distributed capacity, as active making, as value, as resource, as scale, as process, and as responsibility. Drawing on my long history as an observer of children, I anchor this view of humanness in children, and ourselves, as makers: as drawers, story tellers, painters, sculptors, builders, engineers, teachers, writers, care givers, quilters, carpenters, gardeners; in short, as makers and remakers of a human world. (1) 
Carini is clearly a highly skilled reflective practitioner who values multiple modes of meaning-making (or composition). The importance of consciously visually rhetorical forms of communication is evident in the side-by-side methods of example and critique she provides when discussing student work. For example, she chronologizes the typical work of "Iris" as interested in depictions of "strong female figures" (23) and "fascination with memory and interiors" (28) through sample drawings and other images in addition to word-based stories and poems. Carini includes both full color and black and white selections of art from Iris in order to more thoroughly discuss the breadth of composition present in her work. Though Carini does not use a term like multiliteracy, she is yet articulating an appreciation for the multimodal composing processes they adeptly

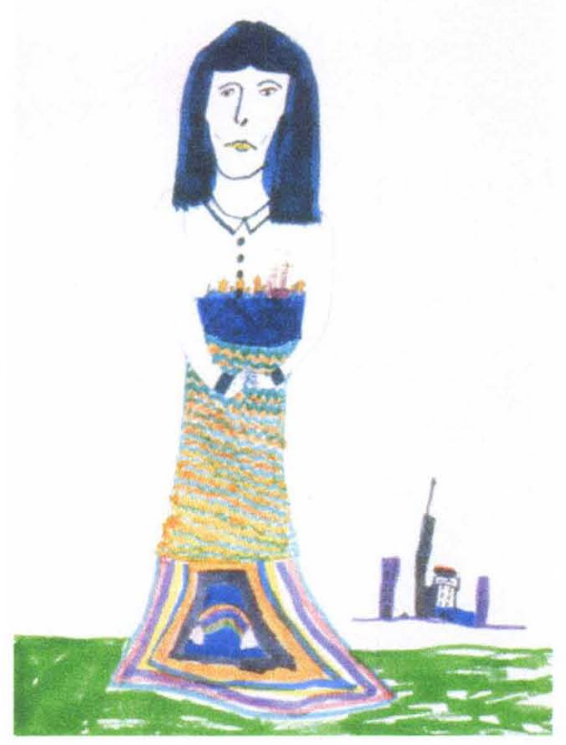
exhibit: "the works children make are both ordinary—and extraordinary: They reflect a widely distributed human capacity to be makers and doers, active agents in the world and in their own lives" (20). This idea of making and being a maker supports the idea of the field of Composition ("Big C") paying attention to composition ("Little c").

This affectionate response to Carini's expressions of word, image, pedagogy and humanness is not fully representative of the manner in which I have conducted this research. The dissertation's purpose of providing "contribution to the field" is a somewhat intimidating one in a climate where so many ideas and concepts have not only 
germinated, but also have been rather well discussed and/or researched. My process in writing and revising my dissertation prospectus was affected by this intimidation factor. I found myself (only after reflecting on comments from readers) fairly consistently attacking other writers and theorists in an effort to make room or perhaps substantiate my own academic pursuits. During the revision, I was shocked at my antagonistic tone. I identified several points at which the reading I was presenting of a particular text was at best myopic and worst inaccurately critical. It took time for me to discover that it is not necessary for me to assert some deficit or lack in the field in order to offer my own scholarship. One of the reasons that my interest has developed in visual rhetoric and culture is due to the work that others have performed. I am not excited by a vacuum; I am excited by works that serve as sources of inspiration.

\section{Reviewing the Literature}

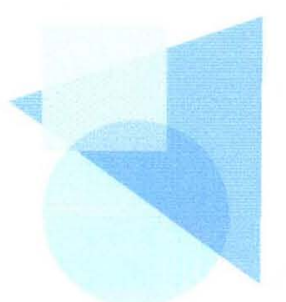

So many FYC students begin their essays instinctively with some provided definition of a term central to their argument. As an instructor, I'm unsure of the sheer number of tomes that I have read that began, "Webster's dictionary defines [fill in any common

subject here] as..." Yet, I find a certain clarity in returning to a root definition for guidance in this beginning, specifically from Aristotle's The Art of Rhetoric:

Let rhetoric be the power to observe the persuasiveness of which any particular matter admits. For of no other art is this the function; each of the others is instructive and persuasive about its special province, such as medicine about healthy and diseased states, geometry about the accidental properties of magnitudes, arithmetic about numbers, and so on with the 
other arts and sciences. By contrast, rhetoric is considered to be capable of intuition of the persuasiveness of, so to speak, the given. That is why we assert that its technical competence is not connected with any special, delimited kind of matter. (74)

This is where I find the initial space, the openness in the field, for the path of exploration I wish to follow: "technical competence is not connected with any special, delimited

kind of matter." The raw material of rhetoric is essentially all material. As a field, Rhetoric and Composition is remarkably positioned to serve as a bastion in the support of independent thought and creative inquiry: our art is in our intuition. I am choosing to apply this artistic sensibility specifically to current cultural given of anime and its potential for instruction. I also find here the root power regarding not only persuasiveness, as delineated by Aristotle, but also what I will label expressiveness. In order to more fully express (and thereby persuade) a particular effectiveness I see in certain anime texts, I will define and use the term "ultrakinetic" in Chapter 3.

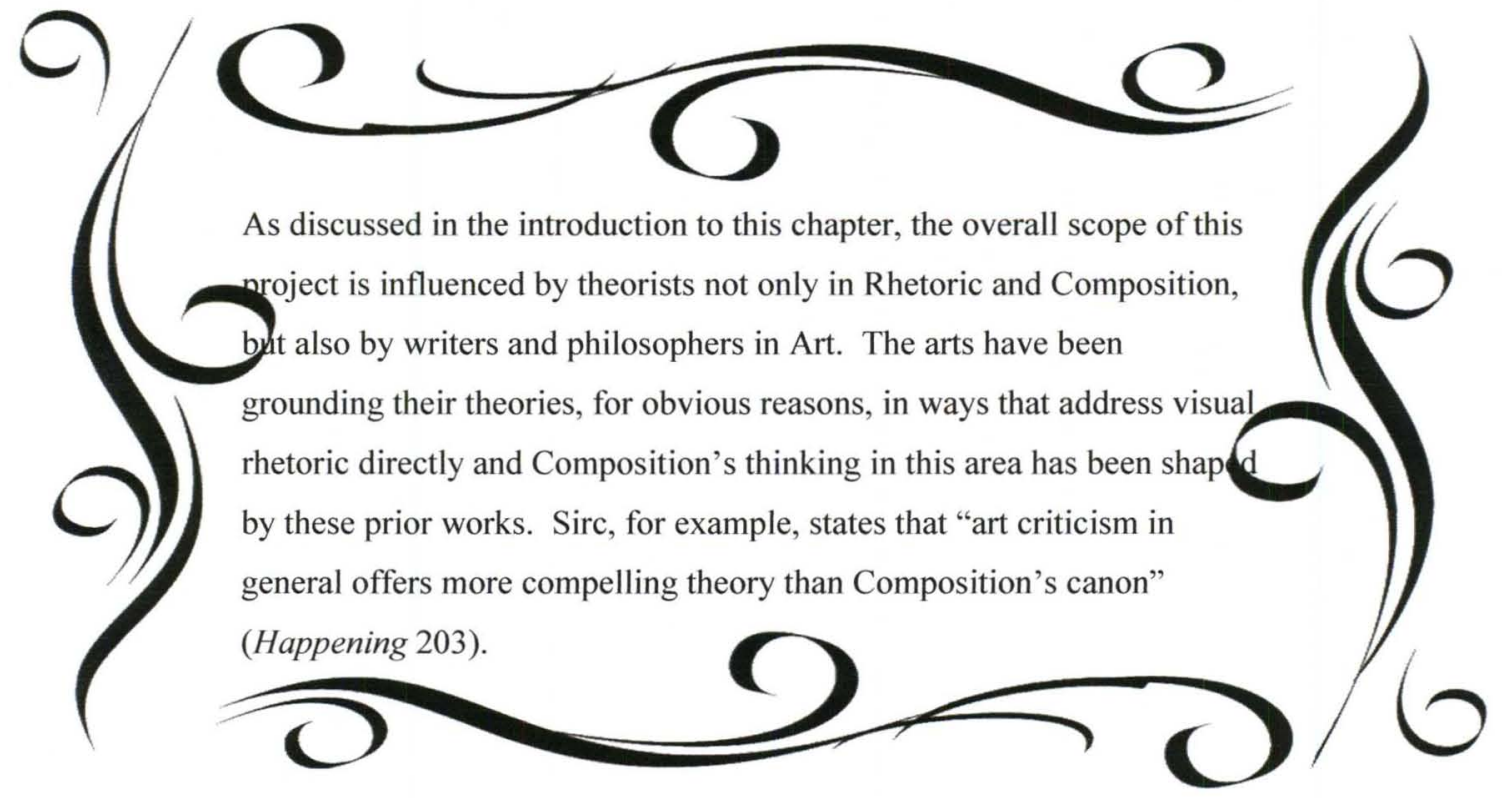


In "Understanding Visual Rhetoric in Digital Writing Environments," Mary Hocks also expresses why composition seeks to understand emerging media in composition by utilizing resources not limited by our specific field of study:

Many teachers of writing who were trained in print-based rhetorics now want to articulate principles of visual rhetoric for our students. We sometimes borrow elements of visual rhetoric from moving image studies and design fields as well as draw more upon the fully visual culture within which our students work, live, and learn...We need to recognize that these new media and the literacies they require are hybrid forms. (630).

Thus, the resources we use when examining, discussing and creating hybrid forms should be a hybrid themselves: an amalgam of the best available theory and criticism in whatever field it may be found. This criticism, in Aristotle's words, would not be confined by "any special, delimited kind of matter" and would be applicable in the broadest, most evocative terms.

Artistic activity is a form of reasoning, in which perceiving and thinking are indivisibly intertwined.

(Arnheim v)

Rudolf Arnheim's 1969 text, Visual Thinking, is a foundational text for visual rhetoric theory and is a suitable initial resource for shaping the consideration of a wide range of criticism that uses his ideas directly. Arnheim situates artistic pursuits in the university as activities that have unnecessarily diminished as "the art student is considered as 
pursuing separate and intellectually inferior skills" (3). Arnheim uses Platonic, Socratic, and Aristotelian perspectives in order to articulate the complexity of perception and thus the intricacy of the arts. Clearly, Arnheim functions as an advocate for art, but his address of classical rhetorical theory is rather consistently even-handed and wellmeasured. His pursuit is exploratory and suggests that early discussion of reasoning, thought, perception and the role of human senses may be more comprehensive or egalitarian than some more recent forms of criticism:

Although the Greek philosophers donceived the dichotomy of perceiving and reasoning, it cannot be said that they applied this notion with the rigidity the doctrine assumed in redent centuries of Western thought. The Greeks learned to distrust the senses, but they never forgot that direct vision is the first and final source of wisdom. They refined the techniques of reasoning, but they also believed that, in the word of Aristotle, "the soul never thinks without an image.' (12)

In Visual Thinking, Arnheim presents a fundamental and precise argument that perception is a form of cognition and, therefore, an intellectual enterprise: "I see no way of withholding the name of 'thinking' frop what goes on in perception. No thought processes seem to exist that cannot be found to operate, at least in principle, in perception. Visual perception is visual thinking" (14). This core, intrinsic value and importance of the visual is one that Roland Barthes will address some years later.

\section{Barthes Is A Visual Thinker}

In "The Photographic Image," one of the essays in Barthes' germinal collection, Image Music Text (1977), the relationship between text and image is explored in terms of the 
way each is linked with the other: "The text constitutes a parasitic message designed to connote the image, to 'quicken' it with one or more second-order signifieds. In other words, and this is an important historical reversal, the image no longer illustrates the words; it is now the words which, structurally, are parasitic on the image" (25). Text and image unite with different possible "degrees of amalgamation," rendering visual rhetoric inextricably joined with verbal rhetoric, though with varying intensity and effect (26). Barthes is considering here headline and article, photograph and caption. When he describes that "every code is at once arbitrary and rational," it is within a specific context (31). Rather than focus on developing a vocabulary of the image like Arnheim, Barthes polarizes the relationship between text and image, a move that others (Kress and Van Leeuwen) will later contest with discussions of the interdependency of text and image in explorations of multimodality. In Barthes, there is a hierarchical relationship, albeit one in which the dominant mode changes: "Formerly, the image illustrated the text (made it clearer); today, the text loads the image, burdening it with a culture, a moral, an imagination. Formerly, there was reduction from text to image; today, there is amplification from the one to the other" (26). It is interesting that text is a "burden" that the image bears, while there can also be "amplification." Burden seems here to be a pejorative while amplification reads as positive, or at least neutral, which signals to me the complexity of the relationship between word and image ultimately for Barthes. Barthes consistently emphasizes the impact of the visual: "If all these signs are removed from the image, we are still left with a certain informational matter, deprived of all knowledge, I continue to 'read' the image, to 'understand' that it assembles in a common space a number of identifiable (nameable) objects, not merely shapes and colours" (37). 
This description is from "Rhetoric of the Image," in which Barthes discusses the content of a single advertisement, as "the signifieds of the advertising message are formed a priori by certain attributes of the product and these signifieds have to be transmitted as clearly as possible" (33). Like John Berger in Ways of Seeing, Barthes spends some time examining a specific advertisement (in this case, Panzani, an Italian pasta) in order to unpack exemplary layers of visual rhetoric. Three layers are defined and explored: "the

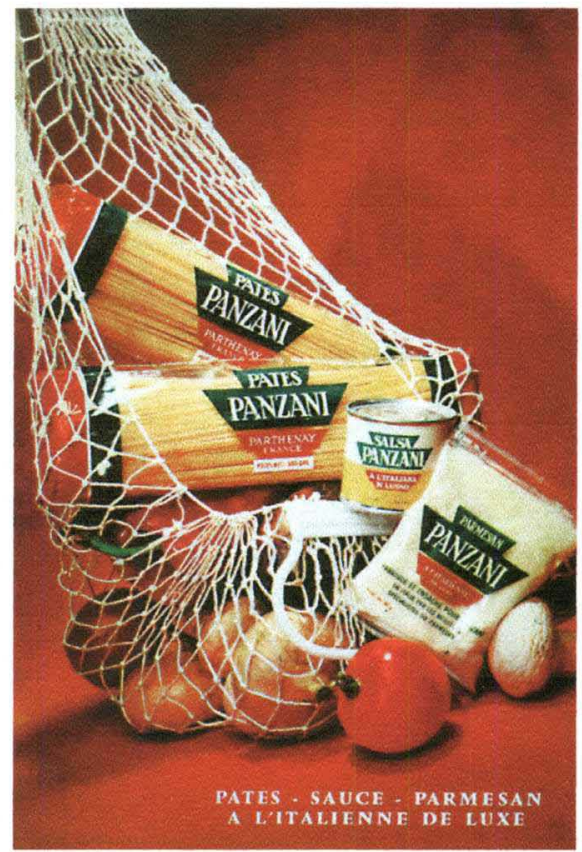
linguistic message" (37), "the denoted message" (42) and the "rhetoric of the image" (46). Each of the three is important and applicable to any discussion of visual rhetoric, particularly those that seek to address the visual in terms of multimodality and hybridity. For example, Barthes introduces his discussion of the linguistic message as a necessary observation of the dominant mode of text and image interacting: "Today, at the level of mass communications, it appears that the linguistic message is indeed present in every image: as title, caption, accompanying press article, film dialogue, comic strip balloon" (38). For most readers, examining text and image simultaneously is almost a given, but remains an activity whose function could bear more study. In Barthes' words: "From the moment of the appearance of the book, the linking of text and image is frequent, though it seems to have been little studied from a structural point of view" (38). This is the first layer explored in the ad: that it contains a caption near the bottom of the overall visual and that several of the internal elements of the image include repetition of the company name, 
logo and product information. This gives way to the next layer of examination: the denoted image, where Barthes further investigates why visual rhetoric is inherently complex. One of the more interesting terminologies is that of the possibility (or, as it proves, not) of a "naïve" image that is not denoted in any manner:

In the image properly speaking, the distinction between the literal message and the symbolic message is operational; we never encounter (at least in advertising) a literal message in a pure state. Even if a totally 'naïve' image were to be achieved, it would immediately join the sign of naivety and be completed by a third — symbolic message. (42)

Thus, as soon as an image is presented as

\author{
The $3^{\text {rd }}$ Layer: \\ The Rhetoric of the Image
}

Barthes' third layer of consideration is the rhetoric of the image, also called 'the third message' and the 'symbolic' or 'cultural' reading of the visual text. This completes his assessment of the Panzani ad and, for my purposes, his most concise treatise on how to interpret visually rhetorical sources. It is also in this area that Barthes calls for further work, noting that there is a lack of specialized metalanguage with which to discuss these matters. Futhermore, it is significant that an image is the product of a particular time, place and understanding; so, too, is the reader of the image equipped to view what is presented in a certain way. Unless, as others have advocated, readers/writers/makers are educated in the new and varying rhetorics that seek to influence our understanding. Barthes asserts that this conversation is truly a meta-level discussion: "Rhetorics inevitably vary by their substance (here articulated sound, there image, gesture or whatever) but not necessarily by their form; it is even probable that there exists a single rhetorical form, common for instance to dream, literature and image" (49)

This is why Barthes is useful to my own study of the ultrakinetic anime text: his core argument seeks to anticipate, even support, the defining of forms of visual texts and the clarification of language with which to discuss such work.

merely-what-it-appears-to-be, it no longer simply is; it is complicated.

Some trends in more recent advertising are antithetical to Barthes' overall argument of cohesive and purposeful representation (specifically in advertising). The contemporary ad often depicts some manner of style over substance in illogical dissociative ways. 
Think of how frequently the product or service advertised is unclear until the end of a commercial or the image presented in print is identifiable as representing the product only through a logo or other tagline. The clarity Barthes indicates is yet retained in the consistent efforts of such advertising to evoke a clear connection between audience and product through emotion. For Barthes, there is a sense of cleverness, as well, in advertising that is a preferred mode.

Take, for example, a more recent

Panzani ad. In 2008 in Prague, the pasta was advertised via dimensional posters displayed in public locations. The paper is the color of uncooked dry pasta and has been physically

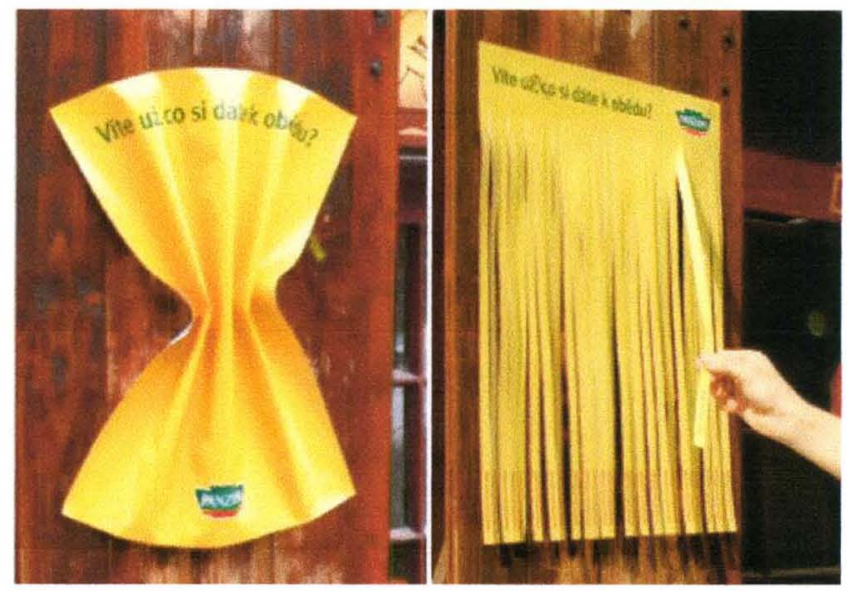
manipulated to resemble specific pasta shapes. In one example, the rectangle of the poster is pinched to appear as farfalle or bowtie, while another paper has been shredded most of its length in long, even strands to signify fettuccini. It is accompanied with the company logo and the question "Do you know what's for lunch?" The inclusion of the tagline and the text-based logo supports Barthes' contention regarding the omnipresence of linguistic messages. But it is also essential to retain Barthes larger commentary that “all images are polysemous; they imply, underlying their signifiers, a 'floating chain' of signifieds, the reader able to choose some and ignore others" (38-39). But if a reader is to have the opportunity to experience texts (like the Panzani ads) at their fullest mode of expression, the reader needs to be well informed (Kress et al might say "literate") regarding the messages coded in the signifiers they choose or ignore. 
Barthes' semiotic deconstruction of the Panzani ad and my subsequent, brief exploration of the more recent example serve as a sort of training ground for directly addressing the complexities of visually rhetorical texts. Barthes unpacks multiple aspects of the original ad, providing a vocabulary for examination of not only the more recent ad, but for all visual rhetoric subjects. Barthes asks, "Is the linguistic message constant? Is there always textual matter in, under, or around the image? In order to find images given without words, it is doubtless necessary to go back to partially illiterate societies, to a sort of pictographic state of the image. From the moment of the appearance of the book, the linking of text and image is frequent" (37-38). In the case of the contemporary Panzani

Another Crinkle: Technology

"The more technology develops the diffusion of information (and notably of images), the more it provides the means of masking the constructed meaning under the appearance of the given meaning" (46).

Barthes anticipates some of the difficulties that contemporary readers face in deducting the layers of meaning in technologically-assisted products. Many in composition (Wysocki, Johnson-Eilola, Selfe, and others) have in their own ways worked toward decoding the new media and providing the beginning of a lexicon of the metalanguage Barthes desires. ad, Barthes is correct: the advertiser adds the text tagline question to make the association unmistakable between advertisement and purpose: you are meant to choose Panzani pasta for your lunch. Would the crumpled paper bowtie have been suggestion enough? Barthes seems to think yes and no. With the presence of the tagline, the "constant" linguistic message again accompanies an image, despite how evocative the pinch of the paper is in creating the dimensional image. The paper is now a piece of pasta no matter what label it carries. Barthes' indications of the power of images would intimate that the tagline is not necessary, though it is expected. 
Ultimately, in his overall argument, Barthes begins to address the philosophical intricacies of text and images, providing an example of Arnheim's visual thinking.

\section{Every image embodies a way of seeing}

\section{Ways of Seeing}

John Berger's Ways of Seeing provides a

natural vocabulary for discussion of the

relevance of investigating visual images.

"Seeing comes before words," this text begins

simply, "The child looks and recognizes

before it can speak" (7). The collection of

seven essays includes three that present only

images. I have chosen to examine Berger in

some detail for several reasons: the influence

of the text for me personally in my developing

understanding of visual rhetoric; the

significant questions raised in the essays

regarding compositional structures; and the

ways in which the text provides not only
(Berger 10)

As an undergraduate, prior to reading Berger, I was already considering some sort of project that would feature intertextuality in some way, but had not yet discerned that the interplaying texts might include something visual. Ultimately, the project I developed centered on 1960s New York poet Frank O'Hara and the relationship between many of his poems and paintings by artists he knew. The project served essentially as a very early draft of what would develop into the M.A. thesis I presented at the University of Louisville. [The same thesis I referenced in Chapter 1 as containing a visual element unwelcome at the time by the Graduate School.]

I subsequently turned toward Ways of Seeing for its familiar, accessible discussions for one of the first writing classes that I taught. The text would serve as a discussion spark and a model for alternative argument construction. Students shrewdly note that the careful arrangement of the images in the pictorial essays is not unlike the necessary organization in more traditional verbal texts. I discuss this further in Chapter 4 when examining the "Anti-Essay" assignment that allows students who have recently read the Berger essays to respond in a similar format.

informational content, but also presentation content in visual rhetoric.

Berger's Ways of Seeing opened my eyes to a new sense of possibility and an interest in visual rhetoric. Though I had long been a devoted advocate of language, reading, 
storytelling and literature, the idea that images and, even more intriguing to me, the combination of words and images together could express and evoke further layers of meaning and responses was revelatory.

If the new language of images were used differently, it would, through its use, confer a new kind of power. Within it we could begin to define our experiences more precisely in areas where words are inadequate. (Seeing comes before words.) Not only personal experience, but also the essential historical experience of our relation to the past: that is to say the experience of seeking to give meaning to our lives, of trying to understand the history of which we can become active agents. (33)

In Berger, I find the same spirit that connects me to Carini's meaning making: Carini also advocates our active agency. The fostering of students as active agents in communication processes should be a consideration when formulating pedagogy. Berger helps shape that pursuit, and is essential for including and addressing visual texts.

The section in Berger that I have found students on the whole respond to is found at the very end, beyond the "List of Works Reproduced": "To be continued by the reader..." placed purposefully and effectively at the top of an otherwise blank page. At the time I first encountered it, this was one of the most palpable examples of visual rhetoric that I had seen. The effective use of whitespace is a point even the most basic design course will specifically discusses whitespace as a key design stress. element. 
In retrospect, it is also significant to me in considering the influences on my theories of teaching and writing that the preface "Note to the reader" valorizes collaborative and reflective writing by describing its authorship plurally (despite Berger's byline):

This book has been made by five of us. Our starting point was some of the ideas contained in the television series Ways of Seeing. We have tried to extend and elaborate these ideas. They have influenced not only what we say but also how we have set about trying to say it. The form of the book is as much to do with our purpose as the arguments contained within it (5).

Berger further relays in the name of the five of us that "our principal aim has been to start a process of questioning" (5). In some ways, this has always been my most ardent academic interest — opening dialogue with peers, colleagues, theorists, students, regarding our most thoughtful reflections on the interconnected and complex web of ideas. Berger's "Note to the reader" (and the printed text of Ways of Seeing itself) seems the sort of context-based example Schon uses to describe the work of a reflective practitioner. Berger's contexts include mainly art and advertising. He is very deliberate in following a holistic approach to these subjects, consistently asking the audience to look not only at the cycle of thoughts and assumptions inherent in the artist, but also their own individual reflective practices.

"The book consists of seven numbered essays. They can be read in any order" (5).

The argument could be made that any collection of essays (or chapters in a larger text, for that matter) can be read in any order. But to what effect? And why is it important to the purpose of 
Ways of Seeing to have such freedom for the reader? These are just a few of the reflections that this practitioner imagines, not only for herself, but also for student readers as they embrace (or struggle with) this visually rhetorical text.

Context, to be sure, is significant. Some of the "discussion" in Berger's work may seem dated to a current audience, particularly some of the remarks regarding gender, consumerism and other issues that are naturally and significantly affected by social environment/context.
"None of the essays pretends to deal with more than certain aspects of each subject: particularly those aspects thrown into relief by a modern historical consciousness" (5).

It is sometimes hard to separate a reader's response to the Berger from this issue or the reality that this text feel (looks) different from others encountered in the classroom. In a Master's level course on the Teaching of Writing [Fall 2008], I assigned the first of the Berger essays as an initial reading-and-response activity, in essence, an introduction to the course. One student dutifully read the essay immediately following our first class meeting. Soon thereafter, she emailed with questions. "I'm not sure why we read this." She was not dismissive or unnecessarily critical of the text, rather, she sought to confirm that her reaction was an appropriate, warranted response. Another student wryly quipped that his initial response was to double-check the syllabus to make sure he was reading "the right thing for the right class." In order to address this subject of student (reader) response, I present and explore several exemplary moments from Berger in the following: 
"It is seeing which establishes our place in the

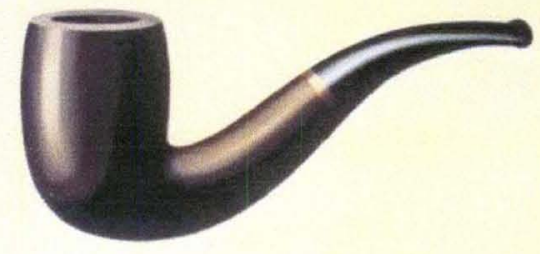
surrounding world; we explain that world with words, but words can never undo the fact that we are surrounded by it" (7).

Ceci nest pas une pipe.

Surrealist artist Magritte's captioned painting labels an image of a pipe "this is not a pipe." In this case, as with the following example of Berger's examination of a Van Gogh painting, sometimes words can "undo" or complicate an image in unexpected ways. In my experience, such complexities spark intense discussion in the classroom.

Another "teachable" Berger:

"This is a landscape of a cornfield with birds flying out of it. Look at it for a moment.

Then turn the page" (27).

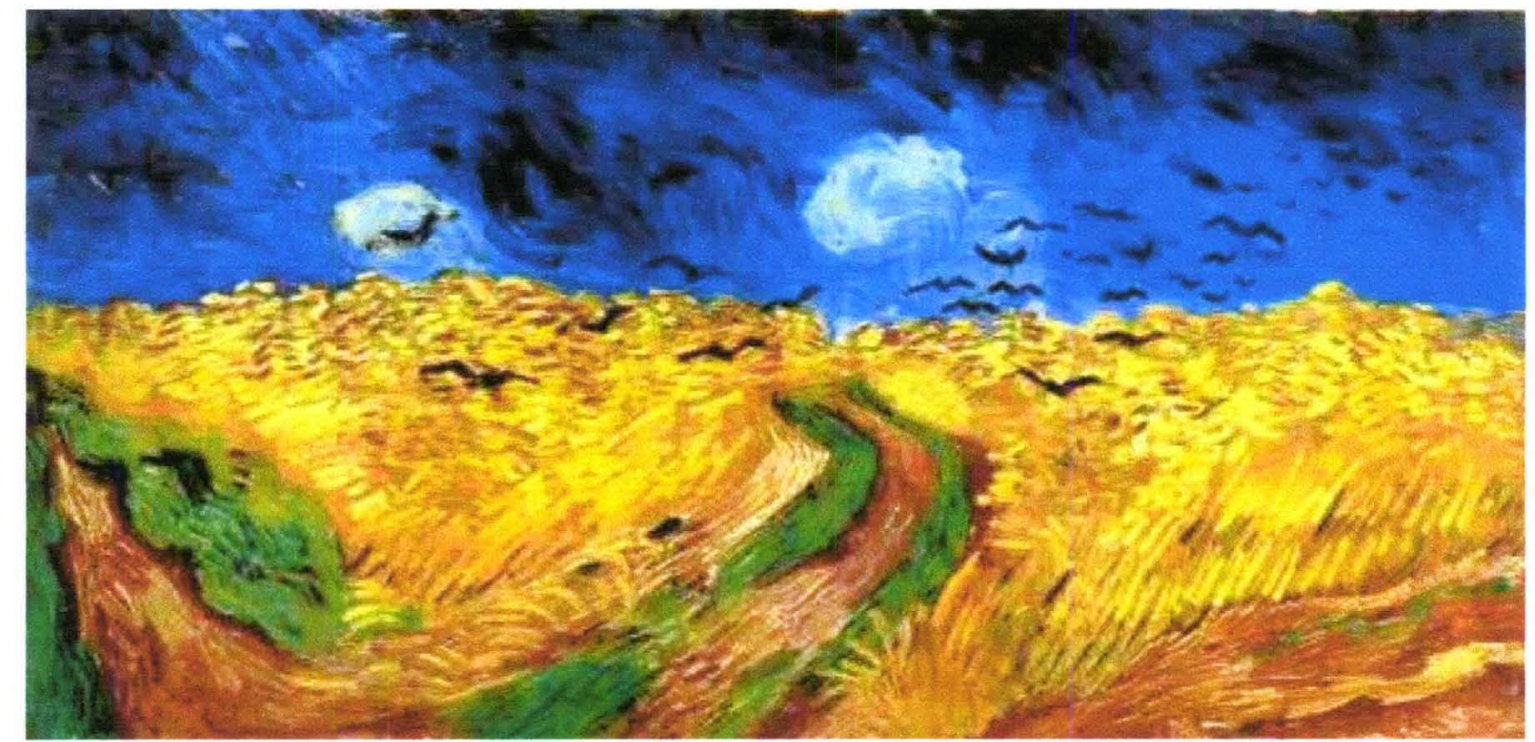

The same image, again, (Wheatfield with Crows, Van Gogh) but accompanied by the

following text: "This is the last picture that Van Gogh painted before he killed himself" (28). The explanatory (commentary?) text is in a completely different font format than the rest of the essay, as if someone had printed this particular information into the book by hand with an ink pen. (Interesting in light of the discussion of "authenticity" that 
Berger offers.) "It is hard to define exactly how the words have changed the image but undoubtedly they have," Berger opines, "the image now illustrates the sentence" (28). This snaps us back with some clarity to Barthes' idea about the frequently dependent relationship between word and image. The caption as Berger presents it may be read as a "burden" on the work of art, as Barthes would term. Consider also the charts presented on page 24 regarding data on educational attainment, art museum attendance, and the attitudes of different working classes [white vs. blue collar vs. "managerial"] toward "a museum" (24). The choice to present such information in a chart format may be an attempt to authenticate the argument inherently (data in a chart may assume the same "holiness" that Berger argues an original work of art retains, as does material in a bound, published edition) or the information is mystified according to Berger's definition by its presentation. Immediately following the second of the two "tables" (as Berger terms them), his discussion of "pictorial reproduction" posits an interesting dilemma, considering the overall purpose of his argument (any argument) is to convince: "like all information, it is either put to use or ignored; information carries no special authority within itself" (24). Then, a mere page later, Berger describes how the medium of film imposes a telling of story (argument)

This overall explication of Berger's text allows me the opportunity to delve into what has been a vital reference for creating applications in visual rhetoric for composition. The brief presentation here of the reaction of students to the text, as well as some discussion of a close reading of the Magritte, Van Gogh, and museum attendance sections helps paint a picture (pun intended) of how Ways of Seeing builds upon the principles examined in the work of both Barthes and Arnheim. Beginning to articulate the interconnection of these texts is an expression of the hybrid theorization process that Hocks calls me to execute when she recognizes (along with Kress and the New London Group) that literacy is now so multilayered, it requires a new approach and that will "borrow elements" from all available resources.

through the segments the camera reveals for how long and in what order. When the same 
painting is viewed live, "the spectator may need time to examine each element of the painting but whenever he reaches a conclusion, the simultaneity of the whole painting is there to reverse or qualify his conclusion. The painting maintains its own authority" (26). Here, then, an image [painting] has authority and is not merely information that can be ignored. But what about the early supposition? I look at it again, within more of its context than I used before:

In the age of pictorial reproduction the meaning of paintings is no longer attached to them; their meaning becomes transmittable: that is to say it becomes information of a sort, and, like all information, it is either put to use or ignore; information carries no special authority within itself. When a painting is put to use, its meaning is either modified or totally changed. One should be quite clear about what this involves. (24) Again, my selection of text automatically influences how my audience may read my argument, but this seems a complex, if not convoluted issue in which Berger provides his own anti-argument. In this case, Berger guides my thoughts in further articulation of some of the complexities of text/image interaction raised by Barthes. Berger is also a resource for providing specific instances of richly layered visual rhetoric for student dialogue.

\section{McCloud}

While Berger's Ways of Seeing is a sort of gateway or primer for students to begin to consider the ways in which visual modes are an important and complex part of communication, Scott McCloud's work is like a second semester advanced course. 
McCloud provides analysis of the effective (and sometimes ineffective) use of visual rhetoric by closely examining his chosen field of expression: comics. In Chapter 1, I mentioned that the McCloud section in Handa's collection Visual Rhetoric in a Digital World stood out from the other essays. In many ways, McCloud would be singular in comparison to a majority of the essays we and our students encounter. He uses his skill as an illustrator to provide both word and image for arguments that examine the interaction of word and image. The effect is frequently astonishing, as what would be for some students obscure description were it presented verbally only, becomes

illuminated.

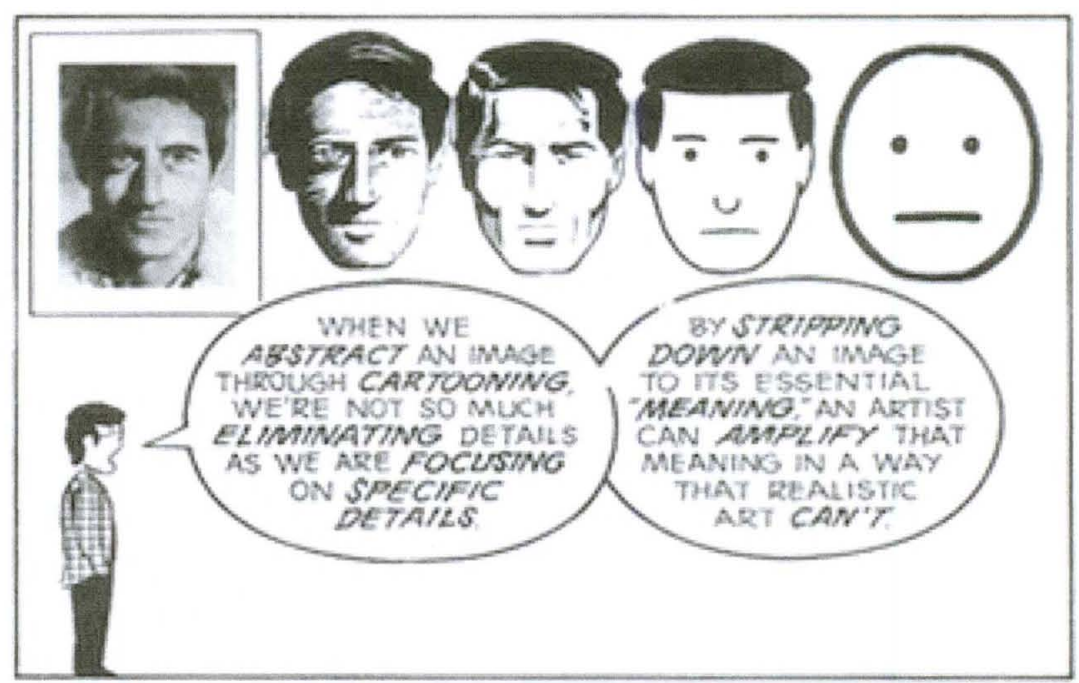

Barthes discusses amplification between text and image; here, McCloud asserts amplification between one iteration of visual and another.

For example, the above panel from Understanding Comics accomplishes in a few square centimeters what might take several more paragraphs of description in word only. For McCloud, the two modes combined are deemed the most evocative; in this he is more in line with Barthes or Berger than perhaps Arnheim. Like Barthes and Berger, McCloud seeks to examine (and, through his comic art, present) the combination of word and image, rather than the examination of one over the other. He does not, however, give any indication that multimodal forms are "easy": 
When pictures are more abstracted from 'reality,' they require greater levels of perception, more like words. When words are bolder, more direct, they require lower levels of perception and are received faster, more like pictures. Our need for a unified language of comics sends us toward the center where words and pictures are like two sides of one coin! But our need for sophistication in comics seems to lead us outward, where words and pictures are most separate. (49). McCloud's terminology of "greater" and "lower levels of perception" is antagonistic, but ultimately McCloud is espousing the vigor possible in the combination of word and image. He also describes the ways in which comics invite the reader in: "We see ourselves in everything. We assign identities and emotions where none exist. And we make the world over in our image" (33). He describes the Japanese comics (manga) technique of "masking" as a consistently effective

McCloud and Berger Share Magritte

McCloud teaches an incredibly clear lesson on perception and the preciseness of language in his allusion to the same Magritte painting Berger references:

"Here's a painting by Magritte called "The Treachery of Images. The inscription is in French. Translated, it means 'this is not a pipe.' And indeed this is not a pipe. This is a painting of a pipe. Right? Well, actually that's wrong. This is not a painting of a pipe, this is a drawing of a painting of a pipe. N'est-ce pas? Nope. Wrong again. It's a printed copy of a drawing of a painting of a pipe...Do you hear what I'm saying? If you do, have your ears checked, because no one said a word" (24-25)

method. Masking involves the presentation of detailed, realistic environments or backgrounds (frequently computer generated) in conjunction with more cartoon-like characters. Thus the figure is a sort of blank slate onto which the reader can transfer her own face: "The more cartoony a face is, for instance, the more people it could be said to describe" (31). Masking is a convention commonly seen in anime styles as well.

McCloud's discussion of Japanese aesthetics can be extrapolated to an introduction to the 
moving version of such pictures: the essence here is a commonality between manga and anime.

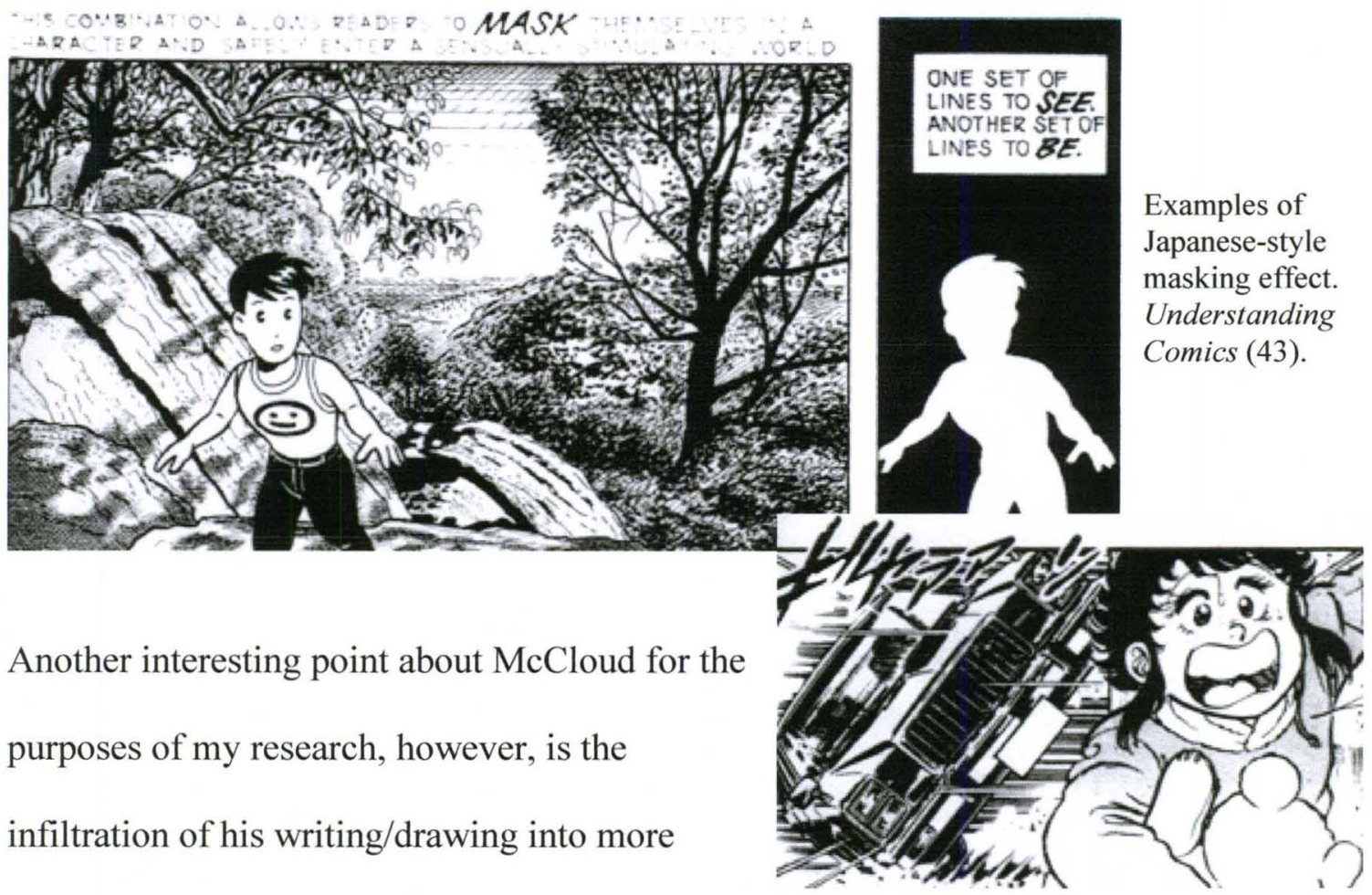

than a few Composition textbooks. A chapter from Understanding Comics is featured in

Donald and Christine McQuade's reader Seeing \& Writing, first published in 2000, now

in its third edition. Bedford/St. Martins sells the text as "the first 4-color composition reader to truly reflect the visual in our culture and in composition." The section that includes McCloud also contains one of the mixed word/image essays from Berger's Ways of Seeing. In 2004, the reader Picturing Texts by Faigley, George, Palchik and Selfe, includes a chapter from McCloud's Reinventing Comics, the follow-up to Understanding Comics. I see the inclusion of McCloud in these readers as a gesture toward recognizing some of the new media as stemming (or existing) in old media. As McCloud (and Barthes) maintain, we have been communicating via hybrid images since ancient history. If we understand the new media as containing or deriving from familiar elements, then 
the reading, analysis, and production of hybrid texts is informed rather than daunting. Though McCloud's intent was clearly to construct a highly accessible exploration of his chosen compositional form of comics, much of McCloud's work is applicable in a broader scope to visual rhetoric in general. As he describes, "communication is only effective when we understand the forms that communication can take" (198). The readers that have chosen to include McCloud seem to have in common the ideal that students should broaden their sense of what sorts of texts deserve analytical address.

McCloud will also appear in Chapter 4's description of assignments associated with visual rhetoric pedagogy. The featured text: 24 Hour Comics.

\section{Mitchell Stephens and the Continuing Saga of Word/Image}

Another shared point of research interest between this study and Picturing Texts is the work of Mitchell Stephens in The Rise of the Image, the Fall of the Word. In their introduction to Stephens, Faigley et al offer that "You've no doubt heard charges that the average American reads less often and writes less well than once was the case. A more sweeping claim alleges that the saturation of images we experience daily makes us think less profoundly" (56). At times, Stephens seems to present an argument that could be used to agree with such a statement (for example, when he bemoans "the fall of the printed word, the loss of our beloved books"), but his ultimate point is to present a level of excitement and optimism for the possibilities of visual media, particularly video.

It is this excitement for visual media that calls for the inclusion of Stephens in this review. In terms of how Stephens helps shape this dissertation, it is his format as well as 
content that is significant. Stephens utilizes the far left and far right margins of each double-page spread of text as space in which to present small images and text commentary. The images are all formatted into perfectly square, evenly size boxes. The text could easily have been placed in foot or end notes. By presenting the margins in this way, however, Stephens gives his reader a designed composition that evokes the valorization of the image in his subject matter without sacrificing the "book" form altogether. In Stephens' words: "The book attempts, in other words, to look without prejudice beyond its author's inclinations, beyond its own form" (xii). Stephens also separates each section with a "splash page" chapter heading that depicts multiple images In comics, "splash pages" are dynamic double-page spreads that usually present a single, visually dense image. stretched across the span of two pages. Berger, McCloud and Stephens combine to not only influence the ways in which we think about visual modes, but also the manner(s) in which we may choose to present our thoughts.

\section{( Sircular Ways of Thinking}

Sirc's work in composition has consistently challenged our status quo and any modes of practice that have lapsed into unquestioned rote. It is with purpose that I title this section Sircular Ways of Thinking, in order to connect Sirc not only to the circular process of reflective practice, but also to Berger's Ways of Seeing and Arnheim's Visual Thinking. Sirc also serves as a bridge between Ways of Seeing and its significant influence for me on both theory and practice and the work of Jack Ramey in his 2000 dissertation. Sirc offers that "the history of our Composition, its failure to exploit its most radical practices, can be read saliently through the lens of the visual arts" (Happening 14). Sirc is a valuable and evocative connector for me of interests in art and classical composition 
within the realm of Composition. Sirc generally seeks to light a fire under the proverbial asses of composition practitioners:

I write out of the lull I feel in contemporary Composition Studies, a disenchantment, which I would locate both in theory and pedagogy. What should be the central space for intellectual inquiry in the academy has become identified as either a service course designed to further the goals of other academic units or a cultural-studies space in which to investigate identity politics. (24)

Sirc consistently seeks to identify opportunity and possibility for the field of Composition. "We have increasingly different compositional means," he describes, "new tools for the mechanical reproduction of texts and an on-going electronic salon in which to circulate them" (36). There may be a lull that Sirc perceives, but there is also potential for dynamism, as evidenced by his suggestion that there is "an unforeseen-use-value" in how we work and that we can focus on ways in which composition is naturally ideagenerative, not necessarily product oriented. I imagine it is this sense of product production that has Sirc unsettled. He seems to think we should be exploring more and that art and artists are good teachers for such an expedition. Sirc selects American "action painter" Jackson Pollock as a model for detailed investigation. He is specifically interested in what Jackson represents in terms of composing processes: "Throughout his career, it seems, there was Jackson's ceaseless interrogation of process, always looking beneath the seemingly random tracing for the underlying tension and a way to capture it" (76). I appreciate the impetus Sirc sees Pollock offering in terms of a way to jump-start our individual intellectual curiosity, our appreciation for the potential of moments, of the 
potential in writing, whatever stage that writing may be in. "If we followed Jackson's choice," Sirc writes, "When we came upon text-as-diaristic-gestures, seeming at first little more than a tangled snarl, our gaze might dwell a little longer on the rupture, not turn back so quickly to overwrite it" (118).

A single term catches my semiotic eye: gaze.

The importance of gaze transcends a variety of genres and modes and has served as a point of interest for feminist critics in particular. As I will later discuss, it will be key perspective in Steven Brown's anime criticism, as gaze has been in film critique and discussion in general.

Here, I choose to connect the concept of gaze with the idea of the screen as outlined by Kress, Brown and Hocks. Both concepts affect our understanding of subjects in relation to subjectivity. For the screen, there is a literal frame surrounding an otherwise limitless combination of modes. The screen is our new measure, our box for containing content, in a similar manner that gaze is both the box and filter with which subjects (usually in film) are viewed.

Hocks evokes just how complex our gaze/screens can be: "The screen itself is a tablet that combines words, interfaces, icons, and pictures that invoke other modalities like touch and sound" (631).
(6) "Imagine, then, Jackson as a subject for process-research: not like the traditional process-subjects, re-tracing stock academic genres, but one engaged in continual experiment with form and material, trying to realize an inner vision" (Sirc 113).
For me, this echoes the experiment

described by Schon and even

McCloud; experiment as reflective practice allows for the exploration of methods in order to achieve the notion of "inner vision" that Sirc describes, but within the writing classroom. 
"We have increasingly different (C) compositional means," Sirc writes, "New Here, the salon encompasses, I think, all tools for the mechanical reproduction of texts and an on-going electronic salon in which to circulate them" (Sirc 36). forms of electronically mediated communication and the multi-modality saturated classroom.

(a)

"What's conventionally thought of as a questionable use of talent turns out to be crucially influential, poetic; what's prized enough to steal is tediously dismissed by the guardians of culture as so much crap academy.

I think here of Arnheim's description of the academic pursuit of art and how dismissed he perceived it to be in the (Sirc 33). actemy.

To achieve composition as a way of being, as action writing, informal writings become a key part of a Happening curriculum, as valid a (non-)genre as the formal essay (Sirc 167).

In many ways, Sirc is reminding us that there is no harm in conducting our work a little closer to home, a little closer to our "real" lives and interests. "I want to bring the field back down to earth," Sirc writes, "to a grounding in everyday life, inflecting our spatial scene according to the rehumanizing tenets of Jackson's Forty-Second Street" (188). For me, this echoes and specifies what Schon has to say about the lack of danger in 
experimentation and reflection: "No move is irreversible. The designer can try, look, and by shifting to another sheet of paper, try again" (158). So, too, in process based (or all) Composition. It's not brain surgery — no one flat-lines if experimentation is performed. Like the designer, the writer is able to write, type, erase, click-of-the-mouse save, delete, combine, and so on. Schon's exploratory experiment allows a reflective practitioner in the teaching of writing to work through a seemingly unlimited variety of possibilities in form, content and execution. At several times, Schon plays on the term "practice" in the sense of it is what one does and the act of repeating an action in order to improve. The way we do better is by doing, thinking, and experimenting through doing again. Schon and Sirc are also in sync regarding how the acts of an individual practitioner can radically affect entire ways of thinking. Schon's final summary on the potential power of reflective practice explains that "the extent of our capacity for reciprocal reflection-inaction can be discovered only through an action science which seeks to make what some of us do on rare occasions into a dominant pattern of practice" (354).

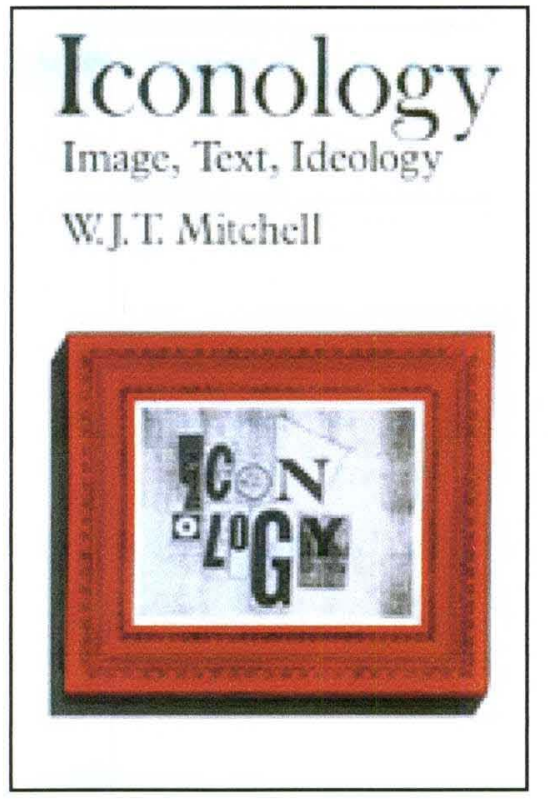

\section{From Text and Image to Imagetext}

Jack Ramey's 2000 dissertation delves specifically into visual rhetoric and composition. In the first chapter, "The Nature and History of the Imagetext," Ramey outlines how his argument uses and diverges from W.J.T. Mitchell's term “imagetext." In Mitchell's lexicon, the imagetext, represented by the two words together, symbolizes the direct, successful relation of the 
combination, while image/text (indicated with a forward slash dividing the two words) equals a "problematic gap," and image-text (a hyphen between the two words) equals "relations of the verbal and visual" without direct combination (2). Ramey uses imagetext, without any dividing punctuation, exclusively, to indicate that all images have an element of text, and all text is visual.

Ramey's third chapter, "Classical Rhetoric and the Electronic Imagetext" applies Aristotle's rhetorical outline of the terms logos, pathos, ethos, and kairos to the World Wide Web. Ramey models the use of rhetoric by conducting a reading of MarineLINK,

Without precedentsetting work like Ramey's, my own text, in this form, and with this content, would not be possible...

\section{Corps}

official website. Ramey concludes that the reading is "an

example of how rhetorical principles can help writers and teachers of writing to understand the rhetorical dynamics of electronic writing spaces so that they can become more effective writers and teachers of these structures and discourses themselves" (114). His subsequent section, "Toward a Rhetoric of the Imagetext for the World Wide Web" examines multiple sites in terms of the elements of visual rhetoric previously defined in his discussion: technical communication, art theory, and document design.

The culmination of Ramey's claims is the substantiation of his theory of language. In "Toward a Pedagogy of the Imagetext," he relays his own personal teaching strategies, 
including handouts, guides, and other methods and materials from his practice. Here we find the details of the theory of visual rhetoric privileged in his reading of the Internet. Terms and other concerns are lifted directly from art and graphic design: figure-ground, color theory, definitions: hue, primary, secondary, serif, sans serif. Fonts appropriate for "display" are differentiated from "textfonts" through not only careful explanation, but also through visual example. "Rule \#4" cautions that the conscientious web author will "always discuss, point to, and label images, tables and graphs" (244). Even as a write this, I know that part of the visual identity I intend to bring to this dissertation includes images that are evocative-sometimes parallel arguments-that I will not so explicitly "label." I admit to having had quite a bit of concern to what the margin-measurers in the Graduate School would suggest. Or, to be more specific, it was my fear that they would require a revision that will, if not negate, at least severely alter the text (Clearly, they did and do not require such a sterile presentation.). But if they had, I would be following Ramey's “Rule \#4.”

Ramey's conclusion is relatively simple, but encompassing for composition as a discipline:

Composition courses and composition instruction may have to change in the years to come to reflect the new ways in which visual rhetoric and verbal rhetoric are merging to create an imagetextual rhetoric for the new millennium...graphic design and the basics of page layout, typography and visual rhetoric could be profitably taught alongside of verbal rhetoric and grammar in first-year composition. Writing now is no longer the 
manipulation of words, sentences, and paragraphs on paper. Writing has move to the screen, a visual space that employs visual elements (251-52).

By referencing the screen in this manner, Ramey evokes the work of the New London Group (Kress, Van Leeuwen, et al) and the logic of the screen.

\section{Multimodality and New Media}

No discussion of visual rhetoric would be complete without the work of the New London Group, Gunther Kress in particular. At a September 1994 meeting in New London, New Hampshire the group met in an initial incarnation to begin discussions regarding the future of literacy in teaching:

We agreed that, in each of the English-speaking countries we came from, what students needed to learn was changing. Clearly the main element of this change was that there was no singular, canonical English that either could or should be taught anymore. Cultural differences and rapidly shifting communications media meant that the very nature of the subject of literacy pedagogy was changing radically (5).

My research question of "What is the impact and efficacy of using anime texts with composition students?" is directly impacted and influenced by this idea of respecting the changing nature of literacy and allowing culture and media to help shape the modes we practice. 
In a profound sense, all meaning-making is Multimodal. All written text is also a process of Visual Design. (New London Group 29)

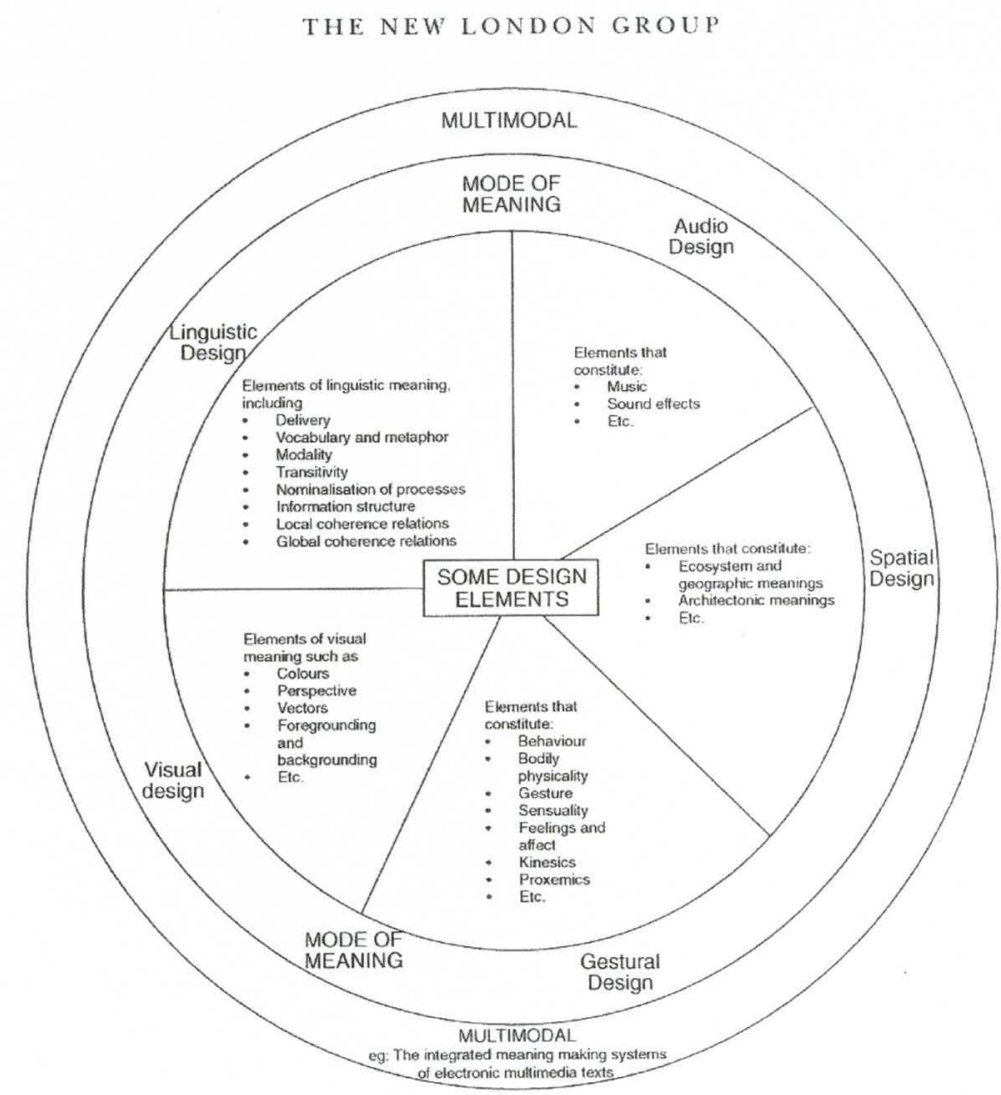

The preceding diagram is an illustration in the New London Group's influential Multiliteracies collection and includes "suggested metalanguage for analyzing the Design of language" (27). By encircling several different "modes of meaning" within a "multimodal" labeled circle, the diagram clearly expresses the overriding proposition for the New London Group that all designed communication is multimodal. The listed "designs" include several facets ("articulated sound, the image, gesture or whatever") mentioned by Barthes (49).

$$
\begin{aligned}
& \text { "text is, for me, a large category" } \\
& \text { (Kress 94) }
\end{aligned}
$$




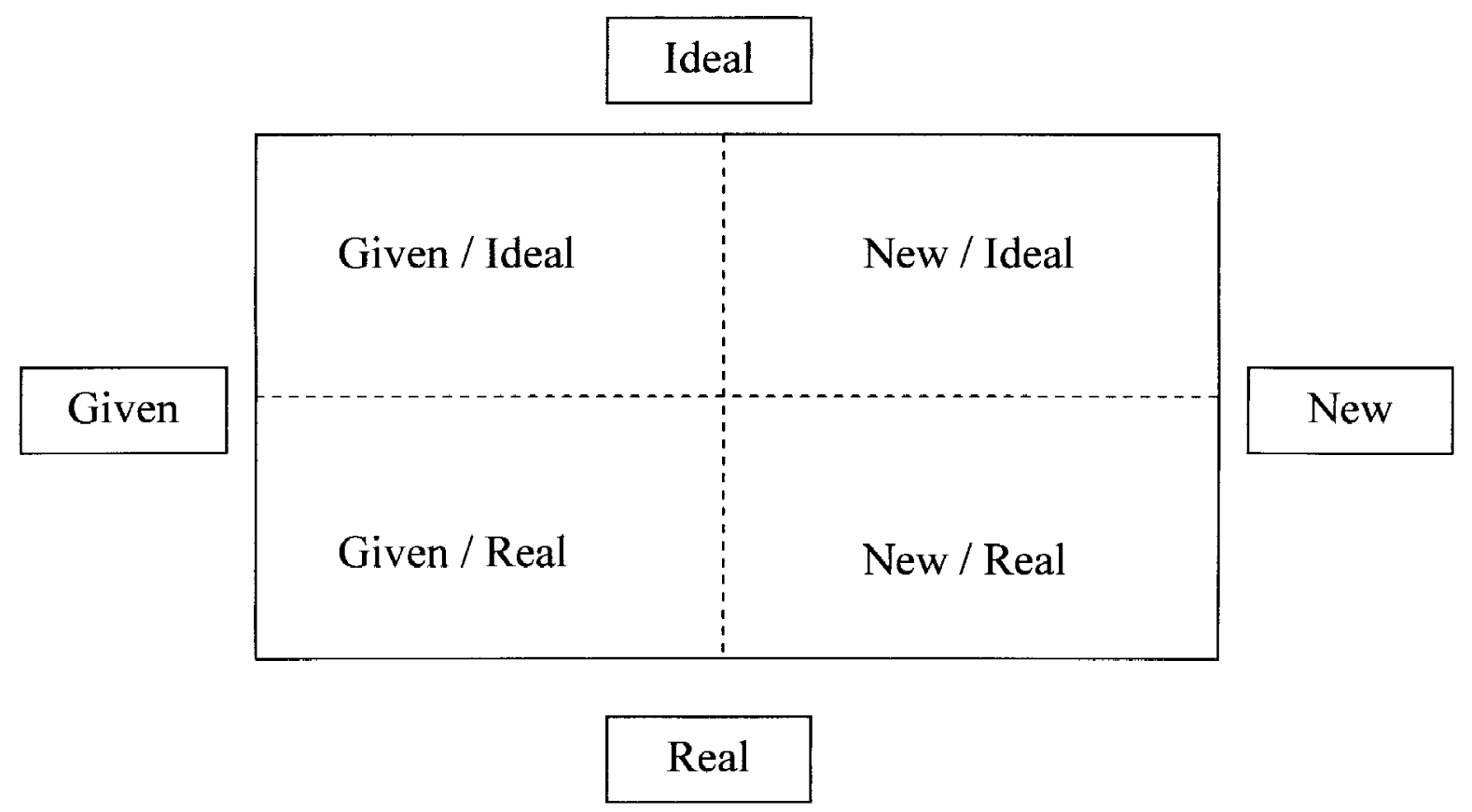

A map by Kress of conceptual spheres of design: literally the location of where a concept will be located in a given design. For example, and image that presents a particular shape in the upper right hand quadrant is highlighting in some way the concepts of both "new" and "ideal." This presents a bare "grammar of the visual" for Kress, who also admits that "other distributions of the space are possible and are in use in different cultures-for instance, a distribution of centre versus margin, where what is central has a different valuation to what is marginal" (200).

In Literacy in the New Media Age Kress describes that a movement from word alone to word and image (and other multimodal forms) necessitates an understanding of change in theory as well: "The theoretical change is from linguistics to semiotics-from a theory that accounted for language alone to a theory that can account equally well for gesture, speech, image, writing 3D objects, color, music and no doubt others" (35-36). In Kress, I also find support for my use of the term ultrakinetic anime text in that he defines the descriptor of text broadly: "I will use the term text for any instance of communication in 
any mode or in any combination of modes" (48). Kress also endorses a semiological approach:

The theoretical change is from linguistics to semiotics - from a theory that accounted for language alone to a theory that can account equally well for gesture, speech, image, writing, 3D objects, color, music and no doubt others. (35-36).

The "others" here can be ultrakinetic anime.

\section{Cinema Anime}

“The visual mode has not been developed into as highly articulated a state as spoken or written language are, for instance, or as they have been developed and conventionalized in other cultures-say, Egyptian hieroglyphics; Chinese pictograms; or Australian Aboriginal visual iconographies" (Kress 194).

Though Kress is directly addressing visual expressions of written language, a larger extrapolation could be made to cultures in which visual forms overall have dominated: for example, Japanese visual culture, particularly manga and anime. In "Screening Anime," Steven Brown describes anime and, in essence, its importance: "contemporary Japanese animation [is] one of the most explosive forms of visual culture to emerge at the crossroads of transnational cultural production in the last twenty-five years" (1). He also connects anime to several concerns in recent composition explorations of multiliteracy and multimodality, indicating that "anime's hybridity of different styles and modes of image making" are part of what makes the work unique, culturally vital and intellectually rich (1). "Screening Anime" introduces Brown's Cinema Anime collection in which the essays are gathered under the umbrella of the expressed purpose or guiding experiment: "What is anime? Anime is so multifarious in its forms and genres, its styles and 
audiences, that one needs to pose the question differently: Where is the anime screen? By asking where instead of what - more specifically, by situating anime in relation to a 'screen' - the question suggests that anime - anime 'as such' — is nothing apart from the spaces of viewing and the technologies of projection (or illumination) that are utilized for the purposes of 'screening' anime" (2). Brown describes and analyzes notions of the computer screen, eyes as screen and mechanization in the anime television series Serial Experiments Lain as a mean of [example of] the importance and applicability of his "where is the anime screen" concept. He further extrapolates that "this collection of essays... is itself a screen" (7). This directly relates to Kress' use of the term screen to describe the "new page": "the task of the reader of the new page," he writes, "and of the screens which are its models, is to establish the order through principles of relevance of the reader's making, and to construct from that" (Literacy 162). For both Kress and Brown, the screen is not just the mechanism of conveyance but also the invitation for the reader to participate in the communication.

There are several interesting themes or issues that are addressed by individual authors in Cinema Anime. Each can be readily associated with composition (both big and little "C") concerns or foci:

"anime's provocative engagement
with the politics of identity" (7).
[Napier, Levi, Takayuki]

CCCC theme in 2007 was "Representing Identities." 


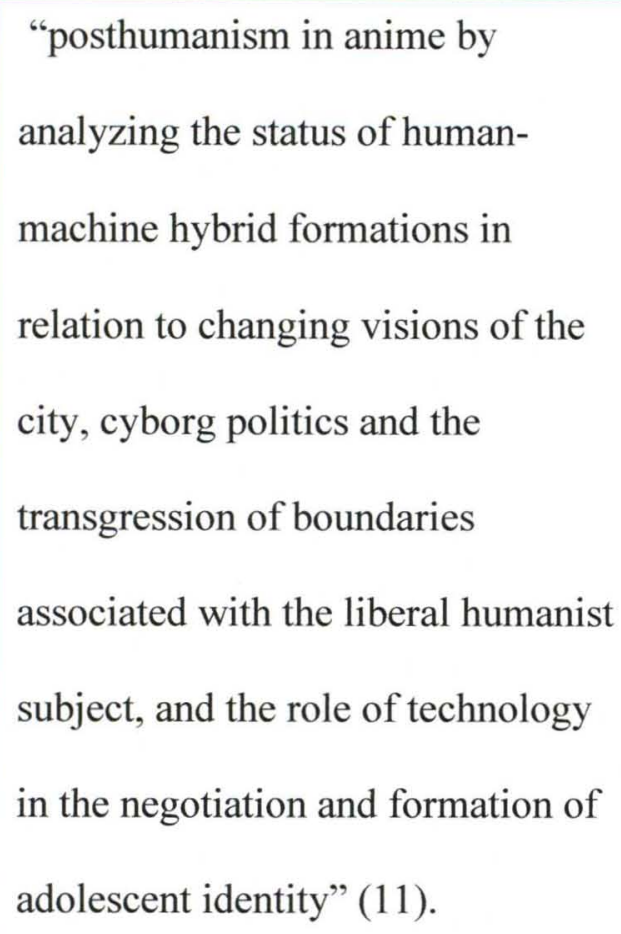

In many ways, composition pushes the limits of communication and consistently challenges our understanding of meaningmaking and expression. There are parallels between the theoretical work in new media studies and this criticism regarding the modes of anime.
Composition has embraced, wrestled, combated with and otherwise significantly explored the multi-layered ramifications of technology on our teaching methods, our practice as writers, the working conditions of our students and, to a larger scale, the cultural environment(s) in which we live/work/teach/learn.

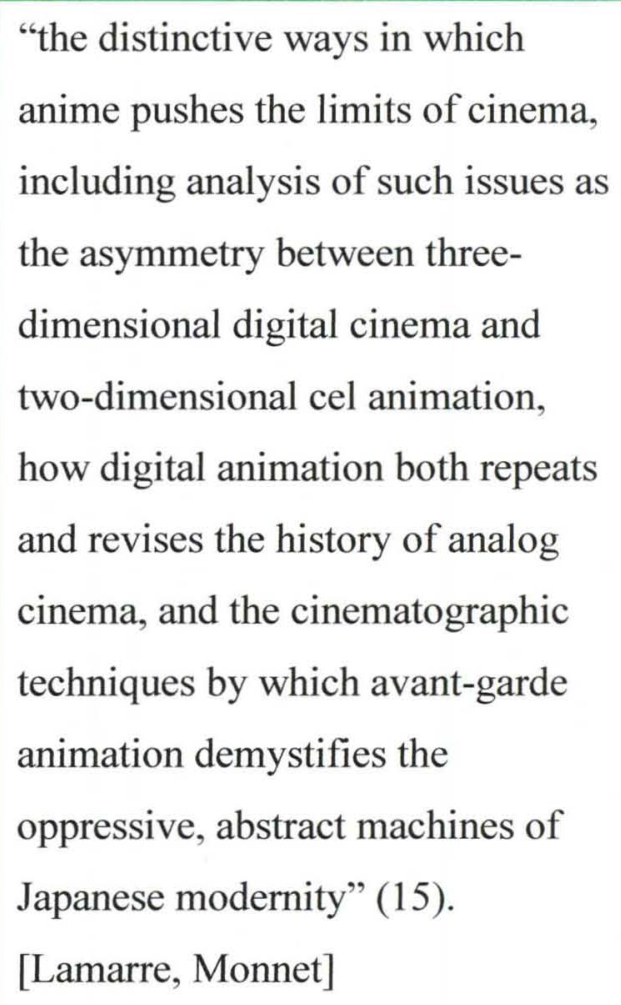
anime pushes the limits of cinema, including analysis of such issues as the asymmetry between threedimensional digital cinema and two-dimensional cel animation, how digital animation both repeats and revises the history of analog cinema, and the cinematographic techniques by which avant-garde animation demystifies the oppressive, abstract machines of Japanese modernity" (15). [Lamarre, Monnet] 
Brown's focus on the screen and Kress' logic of the screen encourage us to more closely examine what is happing within/on the different screens that present information. Such a connection helps highlight the ways in which all of the resources in this chapter interact to serve as the theoretical foundation for the examination of ultrakinetic texts in Chapter 3, in which I continue to address my Schonian exploratory experiment question of "What if we use anime texts in Composition?" They also help frame the anime text visual rhetoric pedagogy toward which this study aims. As described by Hocks, "I want to highlight the visual nature of these rhetorical acts and, conversely, the rhetorical nature of these visual acts as hybrid forms of reading and authoring" (631). The screen is a simple, yet direct and evocative frame for the use of anime [film] in composition. 


\section{Chapter 3}

\section{Anime, Composition and the Ultrakinetic Text}

\section{This chapter will examine the significance of and explore a few examples of what I define as "ultrakinetic" texts. Anime is a mode that provides multiple illustrations of the ultrakinetic concept.}

In the introduction to Eloquent Images, Hocks and Kendrick explain the importance of considering hybrid or "new" media in a holistic manner, rather than as an antagonistic process of deconstructing and polarizing the multiple modes at play:

To attempt to characterize new media as a new battleground between word and image is to misunderstand radically the dynamic interplay that already exists and has always existed between visual and verbal texts and to overlook insights concerning that interplay that new media theories and practices can foster. (1).

It is in this spirit of celebrating the marriage of visual and verbal components that I begin a closer examination of anime, particularly anime that I will define and describe as "ultrakinetic." I define ultrakinetic through referencing the roots of the generation of the term (i.e. why "ultra," why "kinetic"), as well as provide examples of and explore ultrakinetic images. Following this definition, I provide five anime examples and focus

In Chapter 2, one of the design elements presented short blocks of quotation from the resources parallel with a similarly brief response/connection/contextualization. In this chapter, some images are presented parallel with one another for direct comparison, or parallel with commentary text. This design allows the reader/viewer to experience images and analysis as closely connected as possible. 
on different elements of the ultrakinetic within each: Akira, Loveless, Tezuka's Metropolis, Grave of the Fireflies, and Cowboy Bebop.

In Chapter 1, I alluded to the idea that "for composition study, the ultrakinetic anime texts provide familiar (yet unfamiliar) material for analysis that highlights the complexities of multimodality and hybridity." In addition to the temporal visuals, sound, and music typically offered by film in general, anime also frequently utilizes text and both hand-drawn and computer-rendered images. Despite the"Japaneseness" of many anime works, Mark MacWilliams describes anime as a media that on the whole is "culturally odorless, with nothing to keep it from being consumed globally" (17). This is what I mean by anime texts being familiar-yet-unfamiliar. In his description of his own alternative pedagogical efforts, Jeff Rice embraces the effect of the combination of the familiar/unfamiliar: "Hip-hop pedagogy also changes the meanings we would normally associate...Hip-hop pedagogy shifts the familiar into an unfamiliar space by joining unconnected familiar and unfamiliar positions" (92). In this sense, what Rice intends in his hip-hop pedagogy is what I intend with an ultrakinetic anime pedagogy. In this chapter, I define the ultrakinetic text

Patrick Drazen disagrees on MacWilliams' point regarding how Japanese anime should be understood to be, stating that "Anime are, after all, Japan talking directly to itself, reinforcing its cultural myths and preferred modes of behavior... most of the anime you are about to meet [in Anime Explosion] were not intended to be seen by non-Japanese eyes. Some sort of guide is necessary for the uninitiated, to explain the cultural landmarks and injokes: (viii). Drazen is, however, introducing a collection of his own essays, all focused on various components of selected anime presented with a measure of research and a keen eye for context(s), particularly historical ones. Drazen has to maintain the necessity of "some sort of guide," as that is what he is providing. and present a small selection of anime for examination as ultrakinetic. These texts 
present students with the opportunity to investigate media with which they may or may not have some familiarity within a framework of the familiar (film, animation). Subsequently, in Chapter 4, I provide actual assignments and classroom methods indicated by visual rhetoric pedagogy that includes ultrakinetic anime.

Such a pedagogy is exciting since there are no manuals, no guides, no 'rights' or 'wrongs' in this manner of study in composition. There are contexts, imagery, intertextuality and many other challenging concepts, yet no one answer or expression. In "Seriously Visible," Wysocki describes the incredible opportunity in such an endeavor:

The heightened responsibility of constructing meaning out of a text, of not having meaning (the meaning) of a text handed to one by the writer, will encourage readers - students - to take more active roles in their education, to question not just texts, but everything. The implication is that readers — students - through having to grapple with meaning making, will develop 
stronger senses of their varied and particular positions and possibilities

and hence will not acquiesce unquestioningly to other positions. (39)

I do not mean to overstate the proposed effectiveness of using anime texts with

composition students, but I do connect the concept closely with Wysocki's evocation

here of encouraging readers/writers/students to be "active." Though this is clearly

possible with other texts, other methods, other pedagogies, I do want to explore just how

possible it is with an ultrakinetic anime pedagogy.

In The Rhetoric of Cool, Jeff Rice provides

language that I think is interesting to consider in

conjunction with Wysocki's aforementioned

description in terms of how composition can

address specific areas of interest in order to

provide a broader contribution to the field. "I am

looking to generate an alternative approach that is

both critical and performative." he writes,

"Therefore, this is not a book that surveys the

field of computers and writing or new media in

I like the idea of spending some time with Rice's text for two reasons. First, his approach to creating his own pedagogy is one that I can relate to and is in many ways expressive of values that I share as a reflective practitioner. Second, Rice also complicates some of my argument. In his notes he anticipates a possible question regarding his choice to use a more academic tone and form despite his content reflecting some non-linear, avant-garde ideas: "Just because I won't write this book in slang, I don't deny slang's important rhetorical role in cool, particularly how it carries over from hip-hop" (161). One of the key moves I have chosen to make is to move from analysis into performance by presenting this text in a certain format. Rice states clearly that not doing so should not diminish the power of his content. Nor does trying to walk the talk automatically render my argument more cogent.

general; it is instead a book fashioning a theory out of a very specific critique and performativity" (9). Rice, like Wysocki, attempts to qualify his research and experience so that it may naturally provide greater potential applicability. I found this to be a crucial factor as a reader, as the concept of "cool" seemed at first to be a thin semiotic line. One has to dig quite a bit in his text to find definitive statements regarding what cool is and 
why it is important. The term cool for Rice is a Derridian chain of signifiers and therefore convoluted and difficult to reduce to one clear function or expression. Rice does work to ground the concept of cool as a rhetorical act and mode of writing itself. Rice is also interested in contextualizing the rhetoric of cool very specifically within the field of composition: "The missing Fourth $\mathrm{C}$ is not just communication, as George and Trimbur argue, but it is cool as well" (17). The foundation of cool rhetoric hearkens to the birth of modern composition, as Rice cites 1963 and its historic meaning for the field. He also uses many other points of reference in and around the year 1963 for describing and providing further analysis of cool. His discussion, however, is not limited to the $60 \mathrm{~s}$ and applied to contemporary themes and methods, but primarily is concerned with his own classroom practices in writing cool, including reading and writing hypertext.

1963 and Cool All Over

Drazen's history of anime cites the 1963 Japanese television premiere (and in the U.S. shortly thereafter) of Tetsuwan Atomu or Astroboy as "Japan's first animated TV superstar" (5).

A 2005 Newtype magazine article on the anime Cowboy Bebop calls the series "Rebirth of the Cool."

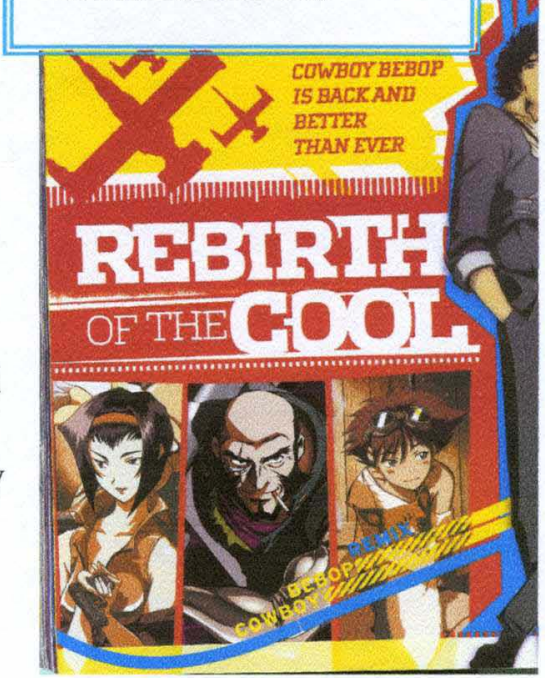

"Hypertext, as a cool form of writing, rejects the outline and functions by way of associations and juxtapositions, not purpose...Students who engage with hypertext, then, should be expected to produce writing far

Hypertext

is cool. (Rice 80) different than what results from an outline. Such expectations may not be situated entirely around purpose or predetermined meaning and should allow for the 'discarded' moment to remain in case it does prove relevant in the mix of things." (Rice 83) 
In some ways, Rice's "the mix of things" is an evocative phrase that at is core defines new media potential for hybridity and multimodality. The final idea that I glean from Rice is not just the power of cool, but the power of cool for pedagogy. And what I call ultrakinetic is definitely cool. The cool of the ultrakinetic text is relatable to the sort of cool that it means to "be cool." Ultrakinetic texts are experiential, frequently inciting an emotional or visceral reaction from the viewer/reader. Ultrakinetic texts are cool in that cool is a state of being.

\section{Defining Ultrakinetic}

The terms ultra-violent and ultra-violence were coined by Anthony Burgess in the novel A Clockwork Orange to describe the preferred mode of entertainment for the main character Alex, and his mini-gang of disillusioned youth. When seeking "a bit of the old ultra-violent" for the evening, Alex is describing acts of violence that exhibit a range of expressions, but are alike in intensity. For Alex, a one-on-one beating of a homeless man and an elaborate home invasion are each "ultra-violent." One is spontaneous and rapid, quickly started and then concluded; the other is slow, deliberate and elaborate. In the home invasion, the gang members wear masks, steal food, destroy property and cheer one another on as they rape and beat the occupants of the house. Thus ultra is not a modifier that merely indicates speed: it is an intensity indicator. In a sense, ultra-violence describes acts of harm that are too much. It is within this definition that I have developed the term ultrakinetic to describe texts that visually present an intensified (ultra) motion (kinetic). In many cases, ultrakinetic texts provide too much information in a single view. The ultrakinetic ranges from home-invasion-like deliberate images that highlight 
the motion through slower methods of display to attacks-in-an-alley-like images that are sped up visuals with layers of picture, sound and word. Akira Kurosawa's film Dreams, for example, presents eight different vignettes, several of which I would term ultrakinetic in the mode of emphasizing slow or deliberate motion. Ultrakinetic describes texts that are a particular expression of a hybrid or multimodal content that invites the viewer to not just see or read the images, but to experience them. In Dreams, Kurosawa draws in the viewer/reader to become a part of his dream experience in the ultrakinetic ways in which he presents the movement within the stories.

I choose to present ultrakinetic, as Ramey does his imagetext, without any separating punctuation in order to indicate that the modifying prefix of "ultra" in this case is inextricably linked with the content indicator of "kinetic." In the concept of the

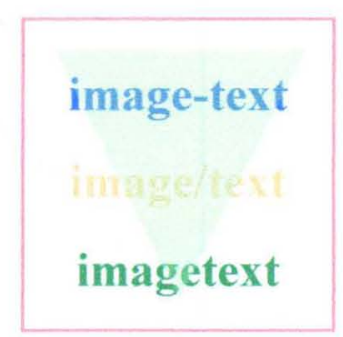
ultrakinetic, the intensified nature of the motion or movement is highlighted. Punctuation would, as Ramey describes in the explication of his choice, dilute the meaning.

In order initially to characterize ultrakinetic, I first turn to a source in some ways quite different from the anime that I will use to explore the term in depth. At the time of this writing, Iran is embroiled in a profound state of political unrest and American news media outlets are dominated by images of the violence and chaos of the protests. Some 
of the images are grainy, unfocused shots taken in the moment by cell phone cameras on site. Others are high resolution crisp videos and still recorded by state of the art news cameras. Not all of these images are ultrakinetic, but many of them are. For example, a moment captured in time, a simple frozen movement is not necessarily ultrakinetic. But a moment in time whose visual highlights the inherent motion of the visual, identifies and emphasizes it, is ultrakinetic. The following image of women placing their ballots in a voting box is an evocative one, but not ultrakinetic by the definition presented. The captured moment is neither particularly still, nor particularly rapid. The scene is visually digestible in that the information available is

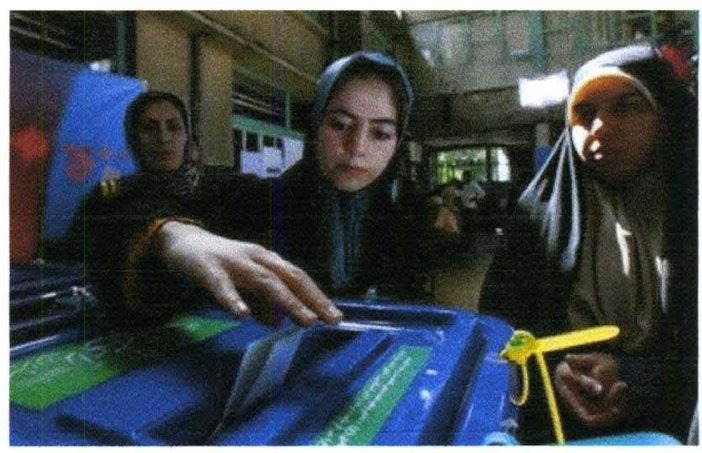
readily consumed.

The following three images, however, each display elements that render the moment inseparable from its movement, even though what we view is a frozen still:

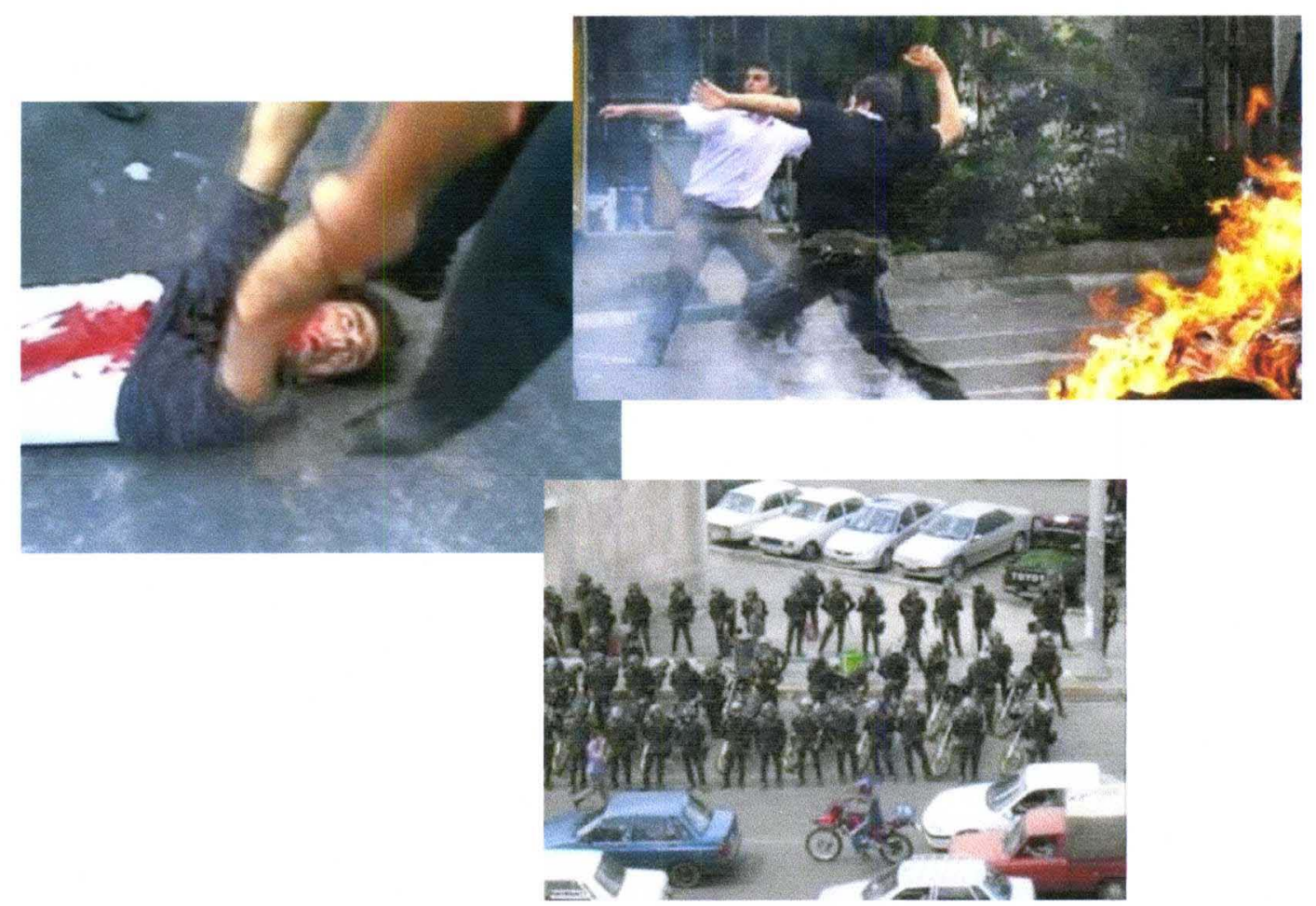




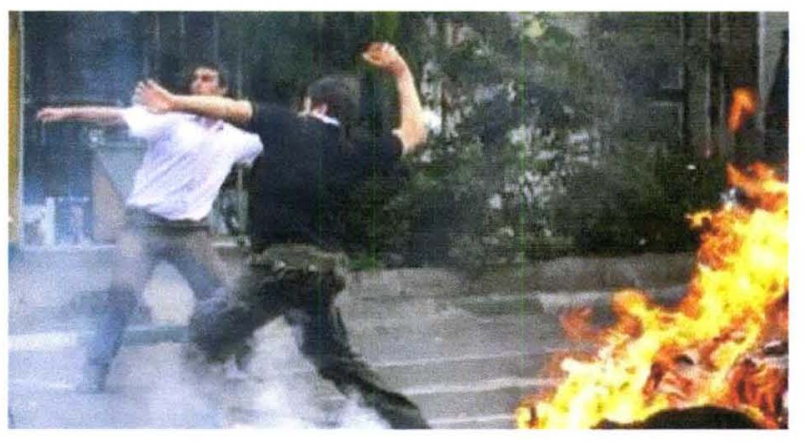

I will begin by discussing the first in this series of images, the one that may most readily equate to the simplest idea of what ultrakinetic means. Two figures in the street, partially obscured by smoke, are each in mid-motion, throwing rocks. Nearby, an unidentified object is on fire with bright flames. The action of the moment is clear, but what makes the image ultrakinetic are the layers of movement: two figures in similar modes, same goal, different stances; smoke billowing in one direction, fire in another; the static element of the pavement of the road stands still while foliage looms in the background, encroaching on the overall scene. Despite the relative simplicity of the image, there is a great deal happening that can be associated with a kinetic descriptor. There is an intensity to this image that supersedes the emotion of the protest and is expressed in heightened movement. The dark figure in the foreground, for instance, is twisting his right knee in a manner that can only be sustained for a moment. In the essence of movement, this is a posture that is in transition from one space, one status, to the immediate next. In art theory, diagonal lines are described as having more interest and as being more dynamic. Such is the line of this figure's leg: drawing our eyes up, toward the activity of his throwing, and down, toward the tumult of the fire. This is ultrakinetic.

It would be easy to assume that the ultra in ultrakinetic is meant to identify rapidity in movement, whereby the above images and their definitions would still stand. Many have noted the ways in which we seem to be quickening in our modes of interaction. Some, 
even, bemoan the effects of the "MTV generation" in which we expect everything to be as quick-cut and flashy as a music video. This is the same collective impatience that leads us to tap our foot at how slowly the microwave is nearly instantly heating our food. In Faster, James Gleick contends that the hastening of our global culture has been steady and somewhat unnoticed: "You hardly perceive the acceleration of art and entertainment: the changing pace of media from cinema to television commercials, which reflect and condition a changing pace in our psyches" (13). Thus the acceleration appears natural, an extension of ourselves and a reaction to our environment. But not everything is moving faster in the new media. Ultrakinetic also encompasses the deliberate stopping, slowing and otherwise non-accelerated presentations of motion and movement in hybrid visual media. For example, the remaining two images in the series I have selected regarding the Iran election protests are ultrakinetic but their kinesthetics are not equal to the "faster" rock throwing and fire in the street. In the second image, an injured man is being dragged away; his facial expression has distorted his features: a result, likely, of pain as well as the way the camera has captured a split second.

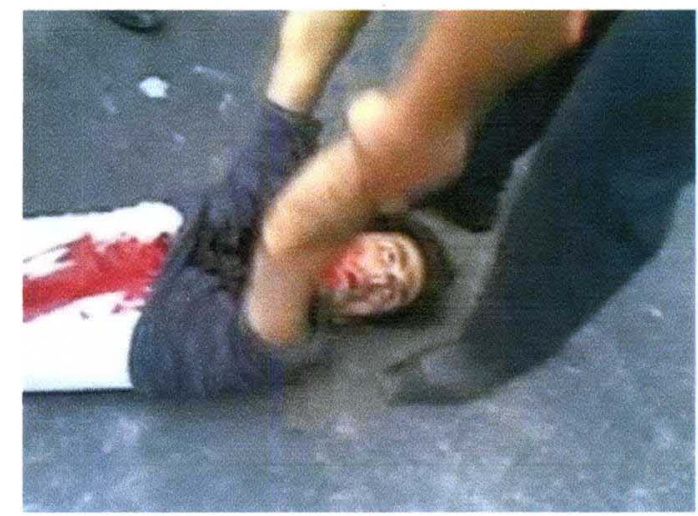
It is impossible to know whether the standing figure is assisting the man and dragging him to safety and medical attention or if this is the perpetrator of the violent act that injured the man in the first place. Each viewer will have her assumptions. It is this layering of meaning and interpretation within the confines of a single, seemingly simple image that helps us experience this image as ultrakinetic. There is very little visual 
information within this particular frame if the fundamental shapes were outlined in comparison to the complicated set of images presented by the rock-throwers-with-fire discussed before. But the visual information that is present is densely packed and evocative: ultrakinetic.

The third and final photo in the series I have selected from Iran is an example of how an ultrakinetic image can emphasize the power and complexity of motion in a moment that appears to be quite still. In this photo, Iranian police stand ready in full riot gear next to a

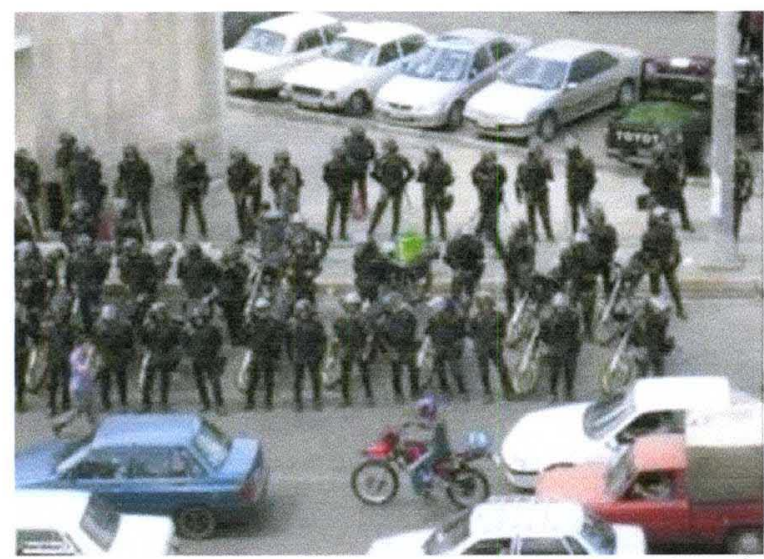
cadre of motorcycles, half in the carfilled street, half on the sidewalk near what is clearly a parking area. The ultrakinetic nature of this image lies not just in the captured motion depicted by the forces gathered at-the-ready, but also in the potential movement suggested by the visual. This is action at rest, burgeoning with all possible chaos and disorder: kinetic activity on the verge. Terming this image (and others) ultrakinetic allows for description and analysis of the layers of rhetorical meaning present in the visual. By spending some time exploring still images, the

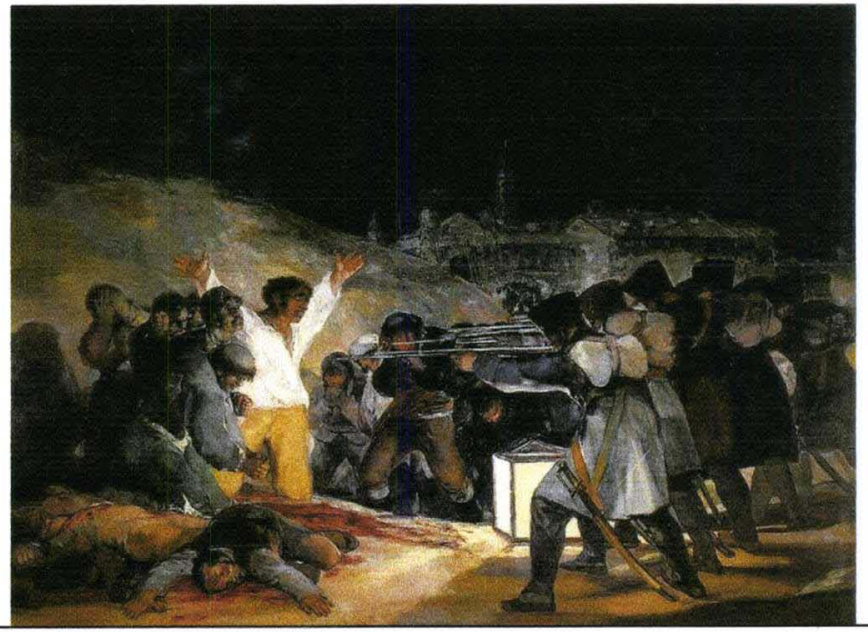

A similarly ultrakinetic image is found in Goya's The Third of May 1808, depicting Spanish resistors to the occupation by Napoleon's army. Here, too, "all possible chaos and disorder." 
definitions of ultrakinetic may be clearer than if we had initially explored modes that do include the complicating element of motion-in-action, as does the following section on ultrakinetic anime.

\section{Ultrakinetic Anime}

As discussed in Chapter 1, I submit that anime holds a particular value for use in composition studies as well as providing

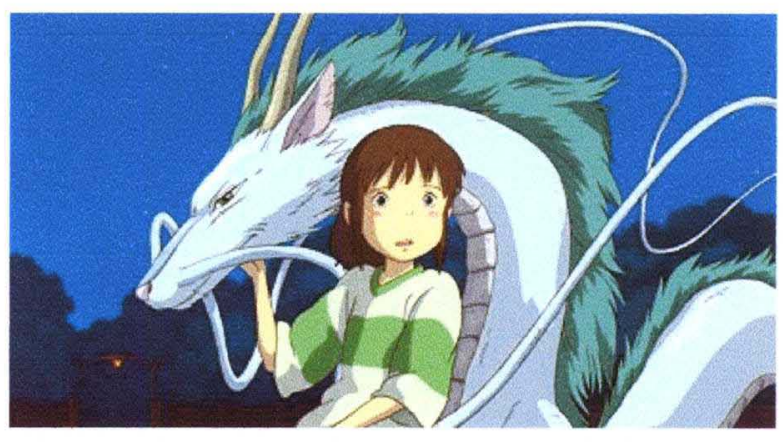

From Spirited Away: Chihiro ("Sen") and the dragon. Even in this still screen capture, the dragon's undulating body pulses with activity, almost as if he is ever in motion, even when we try to freeze the frame.

uniquely effective ultrakinetic images. The full range of ultrakinetic expression is represented throughout the anime catalog, including both markedly rapid and purposefully deliberate incarnations. In Mayazaki's Spirited Away, for example, the ultrakinetic nature of the text is not pace, but rather the visual density and content complexity of the material. Its motion is different from other motions. I identify this difference primarily as existing in the intricacy of the visuals and the ways in which they are set in motion. In more traditional animation styles, the physical environments and settings are fairly static, serving as backgrounds that are visual constants for the action of characters taking place in the foreground. In Spirited Away, the

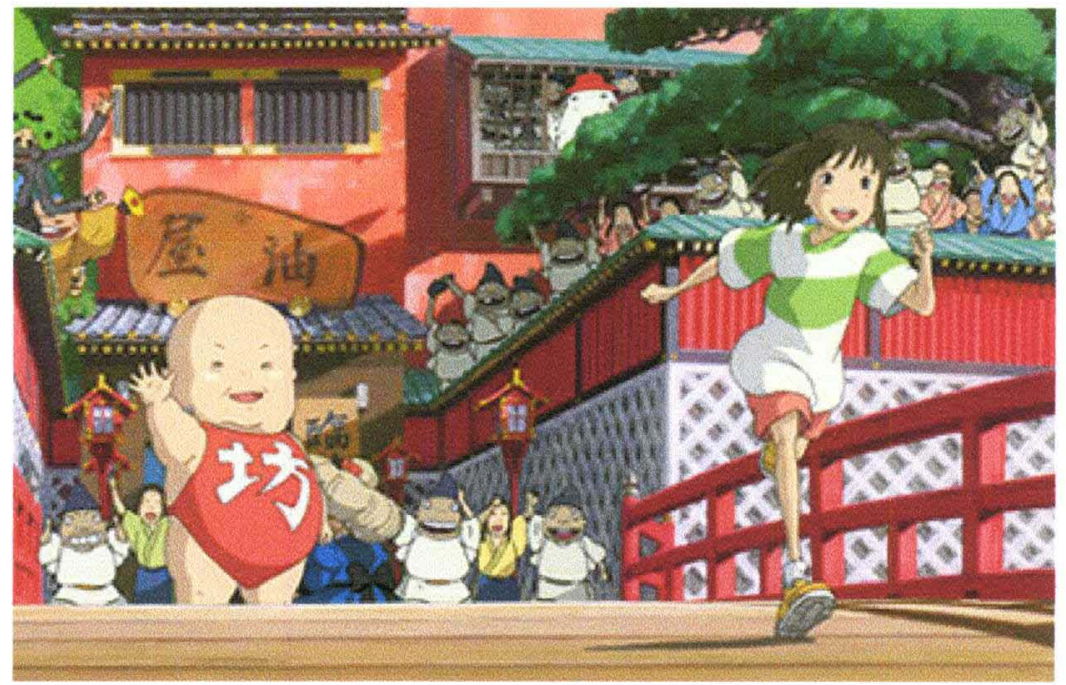


environments are frequently just as active as the individuals themselves. Or, in the

example of Chihiro's exodus from the spirit

bathhouse to return to her family, the throng of

supernatural beings behind her is the environment:

characters, setting and motion combined. This

combination represents a truly ultrakinetic visual that

gives the viewer/reader a reason to look carefully,

closely at all that is happening in the frame.

In the case of the popular television series, Neon

Genesis Evangelion, the ultrakinetic nature of the text

is present even in the aspect of sound. There is even

more to hear than can be digested-skips in time,

temporal space are impossible (too fast) to read.

Much of this is meant to orient the viewer toward the

disorienting perspective of the young main character,

Shinji Ikari, and his struggles to assimilate into the

fantastic circumstances he has been thrust into. The

series is set in a Tokyo of the future where enormous

interactive robots (Evas) are the only viable

protection from imminent threat (Angels).

Shinji, though just a

boy, is meant by his

For an example of non-anime film that layers sound in this manner, see Baz Luhrmann's Moulin Rouge. "Le Tango De Roxanne" is of particular interest in defining ultrakinetic. The sound of various forms of footfall: step, stomp, and slide, combine with an ever-present violin to form this scene's cicadas. 
very genetics to "pilot" one of the Evas. The viewer learns as Shinji learns, experiencing being inside the cockpit from his point of view. Thus, the disorienting flashes of technology, words, the outside environment, the Angel attack, are all seen and heard in rapid-fire succession, each intermingling to create an ultrakinetic visual experience. The viewer/reader is transported through this movement into Shinji's physical position, much like simulation films in which the audience chairs are mechanized to turn and lurch in time with the on-screen images. The Evangelion series helps round out the ultrakinetic definition by providing an example of just how multimodal the hybrid form of ultrakinetic text can be.

\section{Akira: Ultrakinetic Light}

Where Evangelion teaches something about sound in an ultrakinetic anime text, Akira is instructional in the use of light as a layering element of intensity. Akira such an important anime, and an excellent illustration of the ultrakinetic text, that it would be easy to focus on it alone for study. I have chosen, however, to examine Akira for this ultrakinetic expression of light. The film has enjoyed international popularity and is remarkably well-known and enormously influential. One noted reflection of its influence in popular American culture is the direct use of Akira almost frame-by-frame in hip-hop artist Kanye West's video for "Stronger." I will discuss this example further as an indicator of the sort of intertextual analysis in which composition students can participate in an ultrakinetic anime pedagogy. The video for West's song would not be possible without Akira, even if we disregard the lift of storyline content and examine the ultrakinetic presentation of light alone. In the spirit of combining the familiar and the 
unfamiliar, some students will recognize Akira, while others will immediately know Kanye West. Another selection will be versed in film and cinematography (including lighting concept composition). Few will have encountered all of these in conjunction with a composition classroom.

A closer examination of ultrakinetic elements of light in Akira serves as a foundation for looking at the manifestation of the iconic anime in the "Stronger" video. Originally released in 1987, Akira is set in a post-apocalyptic Japan that experiences a nuclear attack in 1988. From the opening frames of the bomb explosion, bright swatches of light fill the screen. As the camera refocuses to display a satellite image of "AD 2019 Neo Tokyo," the viewer's eye at first is unable to discern what the white and red swirl of light is: a brain scan, a diagram of synapses firing, abstract art or some other depiction. Only after the camera zooms in do we see the shapes that are bridges, streets and building from a bird's eye point of view. The light thus serves as an ultrakinetic element that guides the viewer/reader into the environment in which the experience will take place.

The main characters in Akira are part of a Neo Tokyo motorcycle gang and the first scene involving their motorcycles is a key introduction to what viewers may expect in the film regarding the depiction of light and motion. Kaneda (the closest incarnation the story has to an everyman with whom the audience may identify) has the most elaborate motorcycle in the gang. We understand this when, uponstarting and revving the engine, green lightening-like flashes of electricity course around its wheels. As the chase ensues headlights, taillights, and the neon green of Kaneda's bike create ribbons of color behind 
the bikes: motion lines that linger even as the vehicles move out of frame. This is ultrakinetic depiction of light.

Not all light in Akira is bright or neon in nature. The contrast of vast blackness of the bomb impact site signals the viewer that something in this shadow, this non-light, is the heart of the story. The interplay of bright and gloom are also found in the Kanye West video, as mentioned before, seen here in a side-by-side comparison of screen shots from Akira (left) and West's "Stronger" (right).

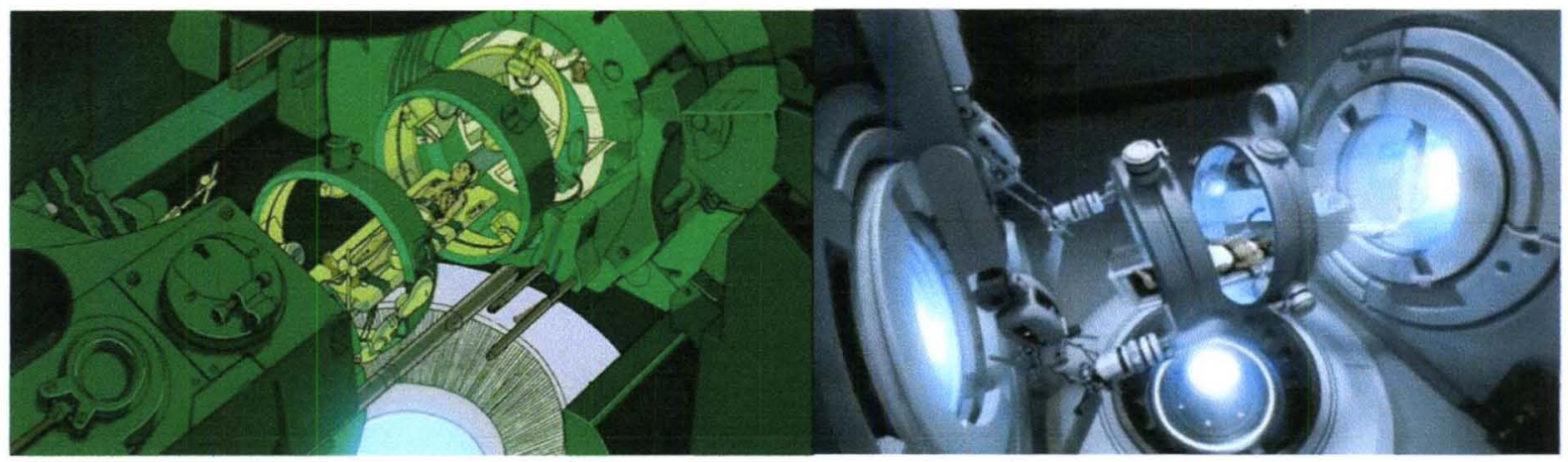

In Akira, the character of Tetsuo has encountered a psychically charged being and his own body composition and metaphysical powers have been transformed as a result. The image of him trapped in an examination machine, bright lights scanning him within a room swathed otherwise in darkness, is repeated in the video, with Kanye West substituted for the Tetsuo character. The machines are similar, but, more importantly, so are the visual representations of light and motion. For the West video, the undulation of the machine's examination provides a visible pulsing to mimic the rhythm of the song. Both moments are ultrakinetic, affording the viewer/reader a multimodal experience in which the image, sound and light combine with layers of reference and meaning. 
In another image from the hospital where Tetsuo/Kanye West is detained, the pulsing is not from the movement of a machine, but from the beleaguered figure moving down a stark green hallway, and the oppressive light of overhead fluorescents beating down.

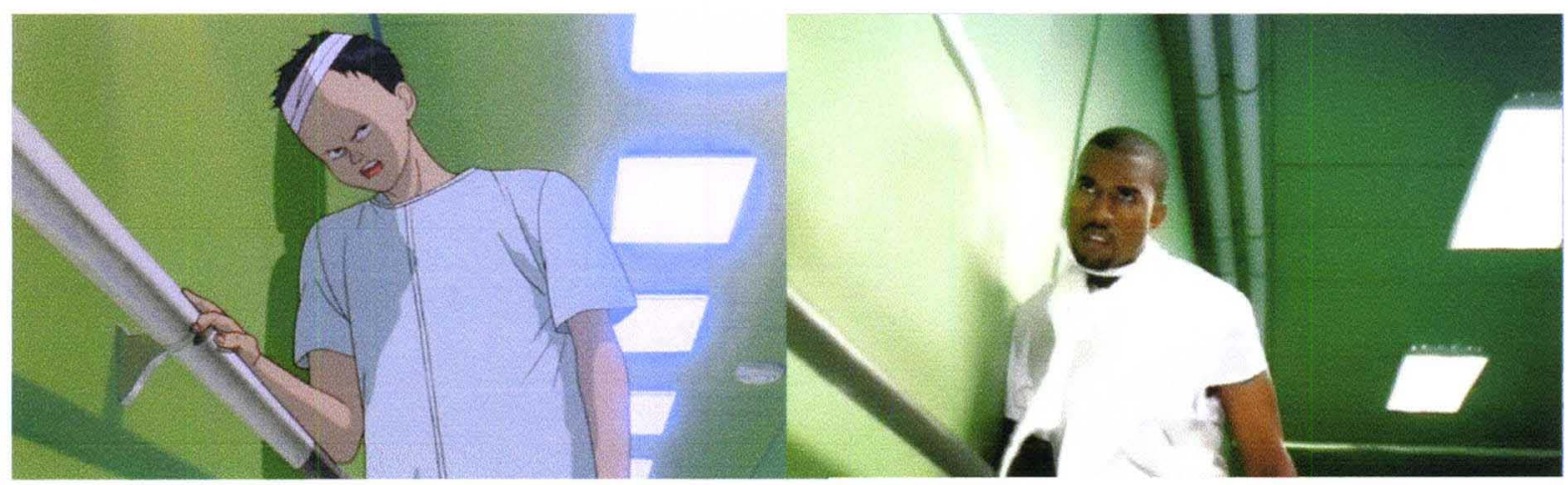

In this instance, West's video misses an ultrakinetic opportunity: with only a few of the fluorescent lights in sight of the camera frame, the lighting effect is not as intense or as emotionally laden as the original Akira frame. The lighting in the West video here is crisp, while the slightly blue, less sharply defined glow around each light in the Akira frame imparts a permeating harshness possible in the anime that is unachieved in the video film.

$$
\begin{aligned}
& \text { The video also lifts the visual of taillights flowing in } \\
& \text { trails behind motorcycles embroiled in an apparent chase. } \\
& \text { In West's video, however, only the red taillight is }
\end{aligned}
$$

manipulated, where the Akira motorcycles streak across the screen with multiple colorful stripes emphasizing the purposeful surge of the machines. In both texts, this technique mimics the common convention in anime and manga of adding "motion lines" to depict movement and emotion. Motion lines in this case are an ultrakinetic element that creates a sense of immersion in the environment: the excitement and/or anxiety of being on a motorcycle chasing or being chased.

Red ultimately proves to be an important shade of light for both Akira and the "Stronger" video. In Akira, Tetsuo's supernatural abilities increase as the story proceeds; each instance of growth in power is indicated by some demonstration of ultrakinetic light. The 
first blinding headaches he receives radiate from his head in shade of blue and purple. As his psychic energy is amplified, so are the ultrakinetic materializations of light. In a scene where Tetsuo is now able to telekinetically control his environment, his entire body is outlined in neon red. In the Kanye West video, a similar image is presented at a particularly dynamic point in the song, where much of the beat drops low in volume and the artist's voice is heard almost a capella. In this case, both texts have utilized ultrakinetic displays of light at their most effective: to highlight a moment of particular importance or intensity. In Akira, it shows the kinetic energy of Tetsuo manipulating his environment, while West's video is building to a different manipulation (trying to attract someone).

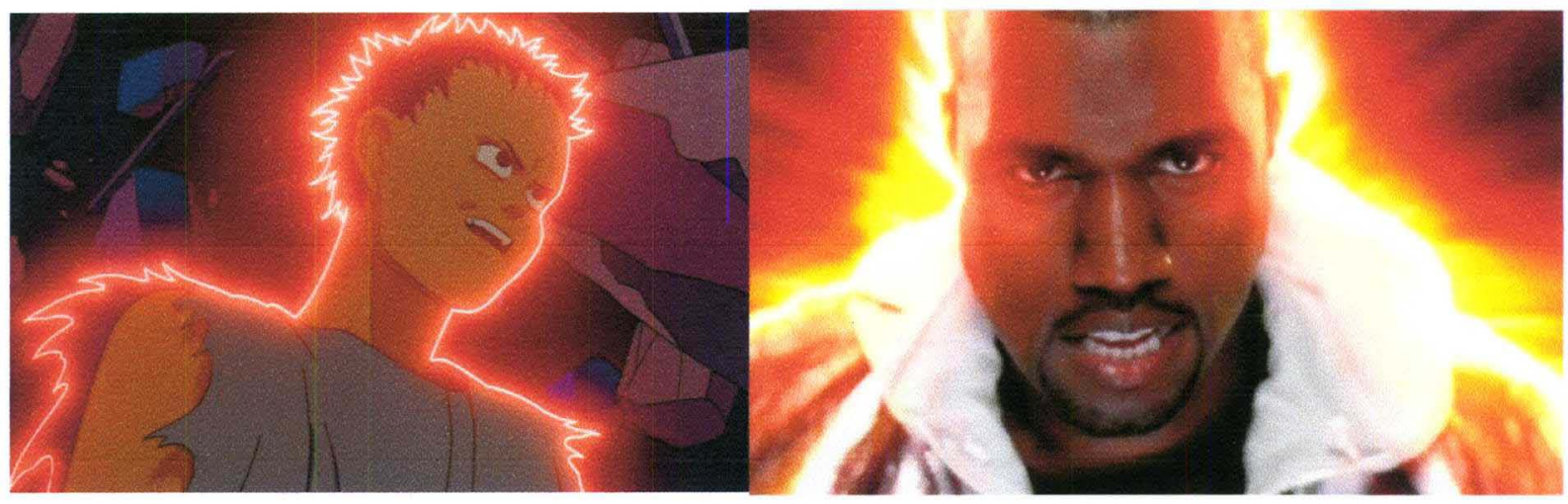

Kanye West's video in many ways seems a loving homage to Akira (not a disrespectful rip-off as some internet chatter may accuse), and is just one expression of the impact and longevity the anime has had on an international level. Now more than twenty years old, Akira has withstood the test of time and belongs not only in discussions of anime or film, but also in any contemplation of sophisticated visual rhetoric. Essentially, Akira could function as an example of any of the modes of ultrakinetic expression that I describe. 


\section{Loveless: Ultrakinetic Words}

Where Akira provides a visual that specifically helps define the use of light in an ultrakinetic manner, another anime will supply a look at ultrakinetic anime and their use of written words within images. The television series Loveless is based in a very

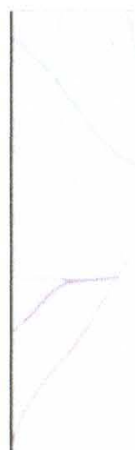

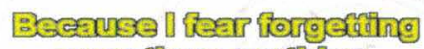
more thean ansythnthe

Screen shot from the title sequence of Loveless, including a subtitled portion of the theme song. The lines in the drawing of the boy emerge and ebb in syncopation with the song, some lines doubling and crossing each other to echo the music in an ultrakinetic manner. different genre from the post-nuclear war vision of Akira. Loveless is shonen ai or "beautiful boy" in theme. Patrick Drazen describes the popularity of shonen ai series in Japan in terms of the romanticism evoked by the stories: "The tendency of these loves to be doomed to

failure, despite their potential for flowering beauty, is precisely what takes them out of the realm of reality and into that of romance and aesthetics. As the kamikaze pilot was glorified for knowingly flying to his death, the doomed boys of shonen ai become beautiful because of their doom" (94-95). (I find it unfortunate that a same-sex relationship is immediately equated with doom, but that is a subject for a different analysis.) In the case of Loveless, the beautiful boys are "doomed" not only by their same-sex romance, but also by a magical set of circumstances that places them frequently in danger. It is this danger, the discussion of it, and the visuals associated with it that are ultrakinetic in Loveless. In the series, metaphysically paired partners share a name and "battle" together, one controlling the delivery of attack spells and the other, the "sacrifice," providing energy for the spells and absorbing physical damage. The main 
character of Loveless, Ritsuka, had a brother who was a sacrifice with his partner, Soubi; their name was Beloved. When Seimei tragically dies, Soubi is attracted to Ritsuka and begins to battle with him even though they do not share a metaphysical name. The magical battles are the primary point of reference for the ultrakinetic nature of Loveless and specifically its presentation of ultrakinetic use of the written word. In battle, spoken words in the form of spells have a literal physical impact: words are ultrakinetic.

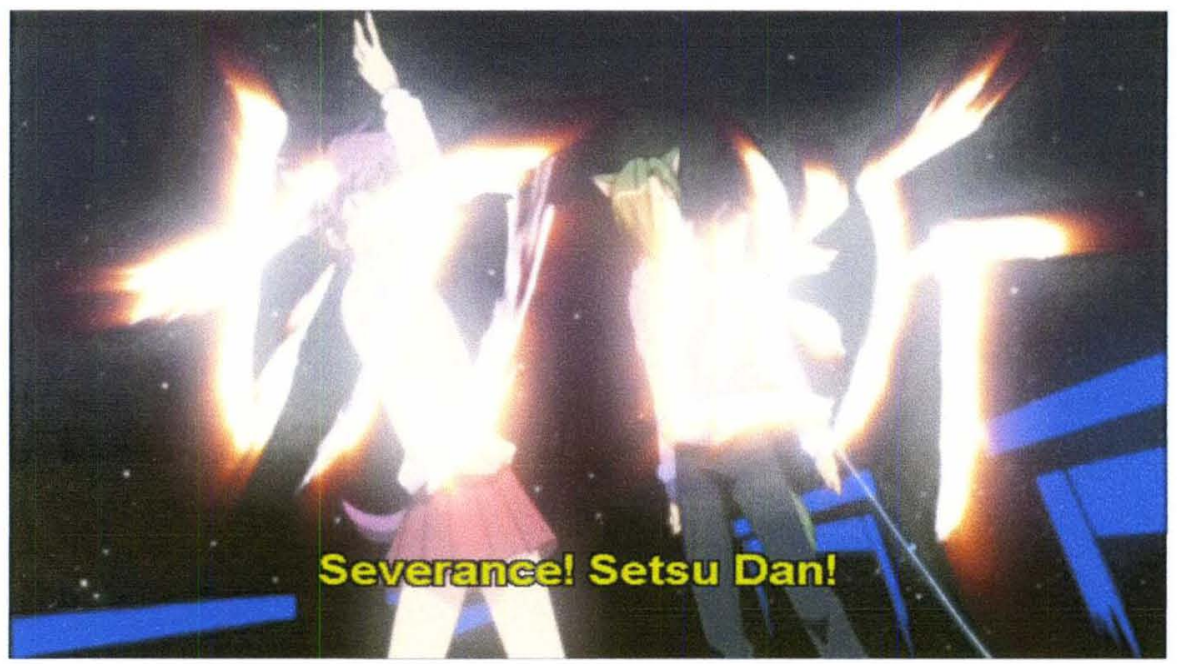

In this frame, a

pair of opponents attempts to sever the connection between Ritsuka and Soubi. The word-spell appears in kanji on screen as the attacker motions the words violently forward. The subtitle presents the kanji's translation in English as presenting phonetically what is being said in Japanese (setsu dan literally means "to amputate"). Battle continues in this manner, with the spells represented ultrakinetically in words as well as in physical manifestations. At one point, for example, collar and chain painfully bind Ritsuka to symbolize the injury he is enduring: the chain glows with the same intensity in which the kanji had flashed across the screen. When
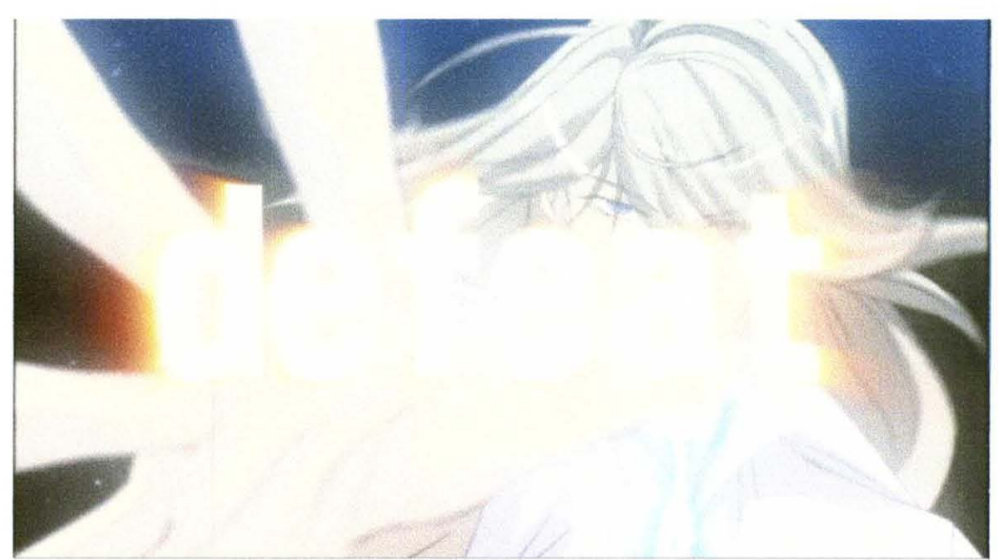
the battle concludes, a similarly lit word emerges on screen as a declaration: this time in English, rather than kanji. Soubi has literally thrust "defeat" onto his opponents in an ultrakinetic application of text.

Frequently, the denouement of a battle session in Loveless will move the visuals from a rapidly changing ultrakinetic series of frames into an ultrakinetic sequence of images in the mode of slowed or still motion. For example, the following two frames are the images that return the viewer (and the characters) from the metaphysical plane of battle back to the school science lab where the confrontation began:

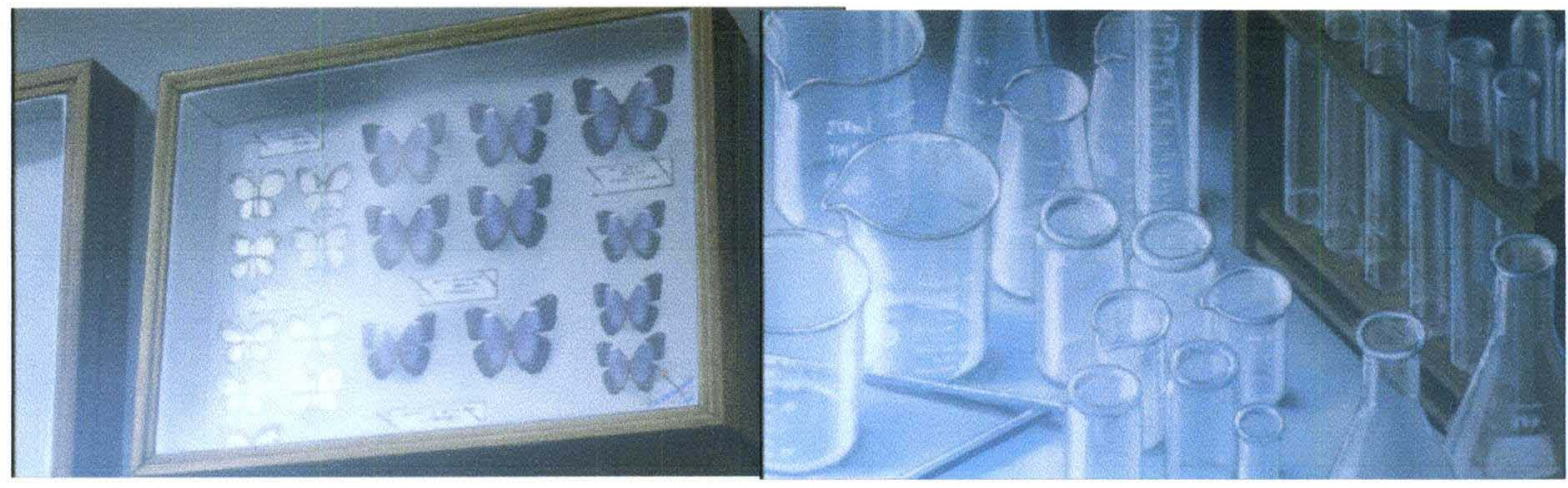

This is in direct contrast to what the ultrakinetic depictions of the action of battle:

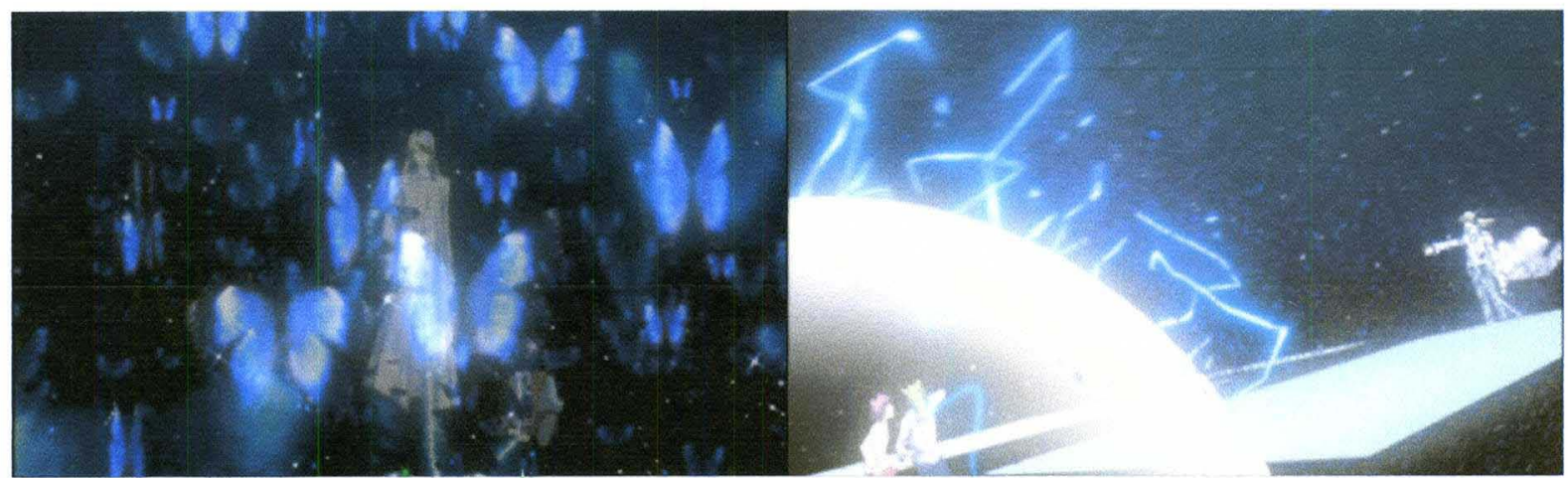
was I able to see in the field of butterflies the small crouched figure of Ritsuka at Soubi's feet. This indicates to me that the faster (hyper?) ultrakinetic modes may provide visual clues and messages that border on the subliminal. 
Here, we see the version of ultrakinetic in which the images are interacting quickly, with more to discern than can be immediately determined by the viewer/reader's eye. This combination of ultrakinetic instances makes Loveless an excellent resource for my proposed pedagogy. I also enjoy the instructional or teachable potential it has for analysis and discussion of other content, ranging from fantasy to basic relationships and social and cultural issues.

\section{Metropolis: Ultrakinetic Storytelling}

Director Rintaro's anime adaptation of Osamu Tezuka's incredibly well-known Metropolis manga is listed among many discussions of "great anime" (Patten, Poitras, Napier). The story is evocative of Fritz Lang's iconic black and white classic of the same name, though Tezuka insists that he only saw a poster for that film and was fascinated by the female robot. Nonetheless, under Rintaro's direction, the anime is a masterful work. I propose that this is in part due to the use of ultrakinetic visual aspects to accomplish the foundations of cinematic storytelling: invoking particular emotions and creating immersive environments and sympathetic characters. Through the intensity of the visual presentation, one of the most sympathetic characters is not even human: Tima, an extremely realistic child robot, who isn't even programmed to understand her identity is anything other than that of a girl.

Ultimately, it is a crisis of identity that leads to Tima's demise and with her a great deal of destruction in the spectacular metropolis.

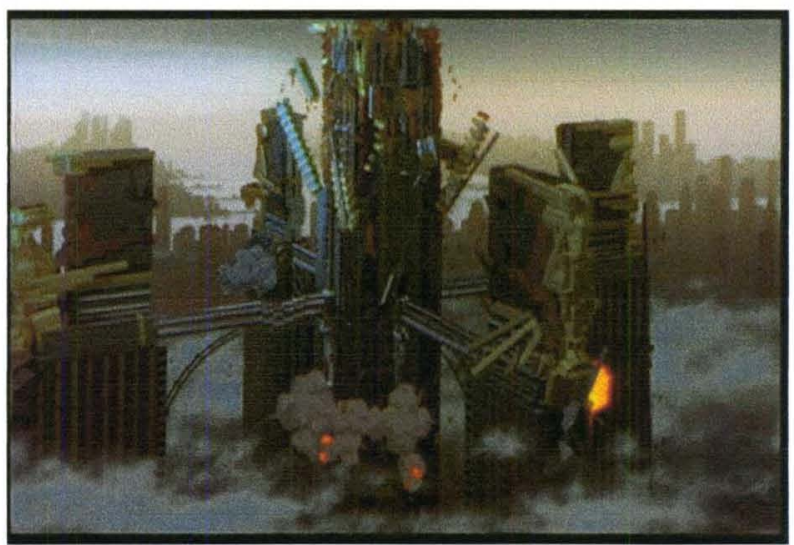


Tima's final moments are the focus of this analysis of the ultrakinetic in Metropolis. Shattered emotionally by the lack of understanding of her own state, Tima begins to manifest her pain in the complete demolition of the great building that her creator erected in monument to his own achievements. The walls give way as Tima crumbles in mind and in body. The cascading pieces of the structures fall elegantly in ultrakinetic

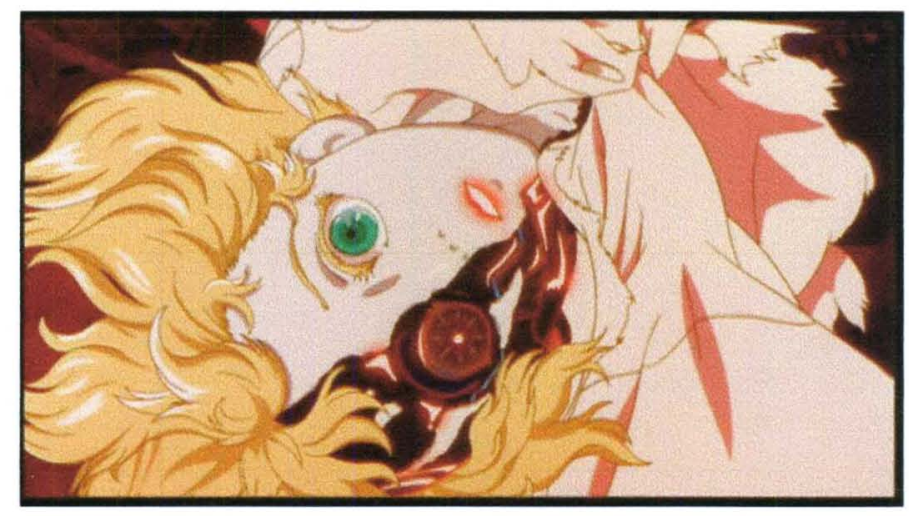
motion in frame after frame. Tima, as well, remains inexplicably beautiful even in her damaged condition, half of her robotic core exposed. Though everyone is in danger at this point and Tima is the impetus for the devastation, the viewer/reader does not blame her: she is the victim in this circumstance and the ultrakinetic storytelling has enabled this to be clear.

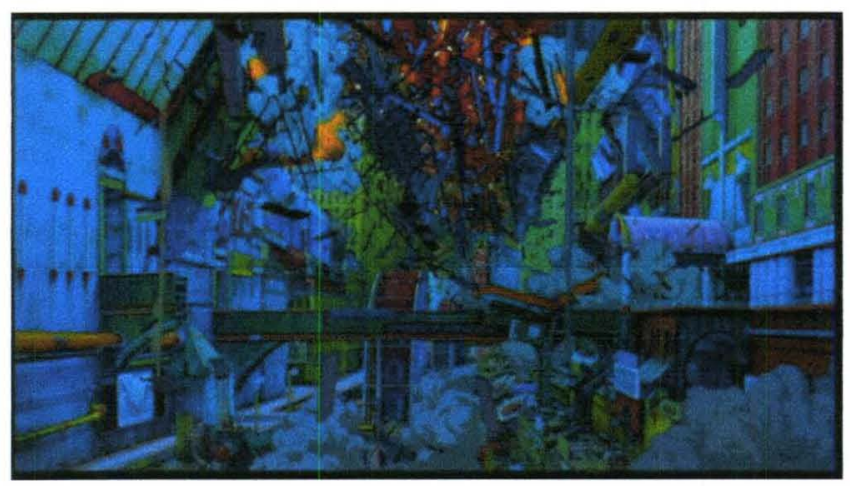

Another unique ultrakinetic method of storytelling found in the music that accompanies this closing sequence of the film. Ray Charles croons "I Can't Stop Loving You" while the buildings fall. In some ways, this prepares the viewer/reader for the idyllic peacefulness that the final images provide. The colors are brighter, sunlight: soft and soothing even 
though they blanket a shattered city.

A flock of doves rises from the

debris, a sign of life and love

continuing on. Without the

ultrakinetic layering of what is

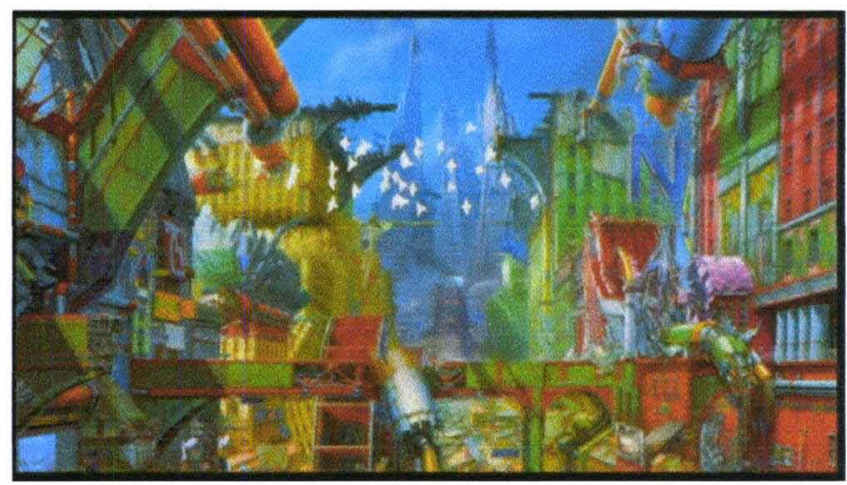

otherwise a strange choice of music for such a moment, the story would be different. The ultrakinetic elements allow the ending to be an experience for the viewer/reader in which image and song create an atmosphere that bears further investigation. In my experience, first-time audiences of Metropolis are inspired to discussion and additional interaction regarding the text. This is the sort of reaction a pedagogy of ultrakinetic anime aims toward.

\section{Grave of the Fireflies: Ultrakinetic Stillness}

In The Anime Companion Gilles Poitras states that "all parents should seriously consider watching Grave of the Fireflies with their kids as an opportunity to discuss what war and its effects on civilians is like" (12). Based on the novel by Akiyuki Nosaka, Grave of the Fireflies was originally released in Japan in1988, but did not arrive in the U.S. until 1992, perhaps because of its unflinching depiction of the devastation of American actions during World War II on the non-military residents of Japan.

The story is told in flashback after the initial scene. Sepia tones throughout add to the

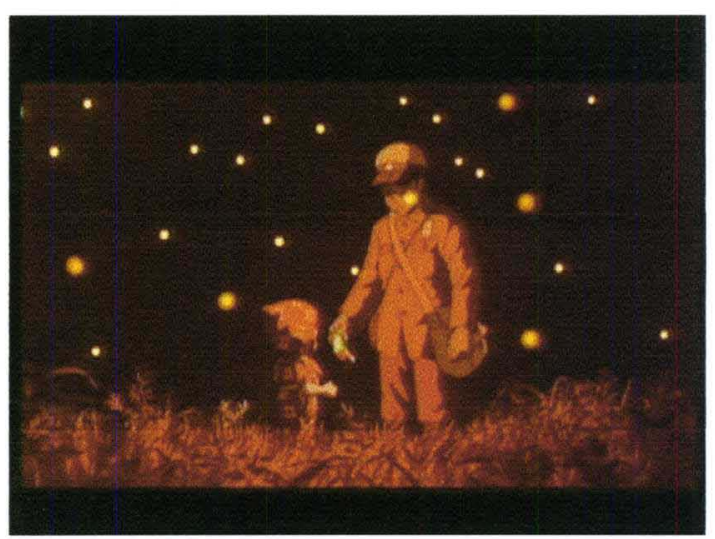


nostalgic sense of moments and movements gone by, already long lost. We know from the beginning that the boy dies alone. "September $21^{\text {st }}, 1945$," the narration softly introduces, "That was the night I died." Seita’s spirit joins that of his sister, Setsuko, in a field surrounded by fireflies as her voice echos, "Why do fireflies have to die so soon?" He hands her a tin of fruit flavored hard candy, a luxury that was a source of comfort for her throughout their hardship together. It is a simple moment, composed of gentle movements, and it is in this an ultrakinetic visual of stillness. This is a truly "slowed" mode of the ultrakinetic.

The candy tin figures prominently in another significant example of ultrakinetic stillness in Grave of the Fireflies. Setsuko lies near

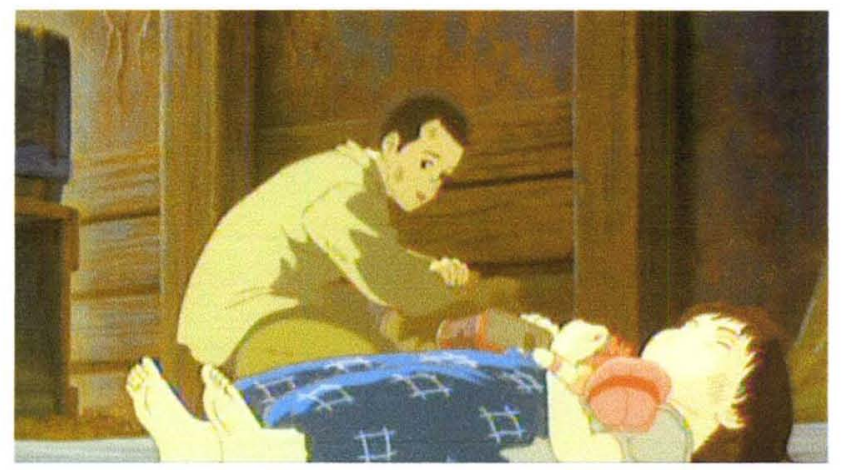
motionless on the floor of their shelter, a ragged doll cradled under one hand, the candy tin in the other. Her only movement is a gentle rolling of her mouth, as if she is attempting to make one of the fruit drops last as long as she can. The reality is that the drops have been gone for some time; she has replaced them with a few marbles and her movement is rolling one of the marbles around her tongue. The ultrakinetic stillness underscores the emotion of this sweet, pitiful gesture just an instant before Setsuko succumbs to illness and famine.

The anime film Blood: The Last Vampire at first glance is entirely dissimilar from Grave of the Fireflies at first glance. It depicts an unrealistic world in comparison to Grave's stark historicism and does so in an entirely different ultrakinetic style. Where Grave of the Fireflies is stillness, Blood is utter chaos. But ultimately this is a film about war and violence - all too human themes found in both anime. The closing titles of Blood show a montage of soldiers, tanks and other vague images of battle. This distain for all things associated with a contemporary military is present throughout, even in what methods of interaction are viable. In Blood, guns don't work on the vampire enemy: only the sword. In an ultrakinetic anime pedagogy it would be interesting to pair Blood: The Last Vampire and Grave of the Fireflies in a seemingly disparate double-feature. 


\section{Cowboy Bebop: Ultrakinetic Jackpot!}

Thus far, we have examined ultrakinetic anime styles that accentuate not only an array of movement, but also sound, light, written words, storytelling and stillness. All of these ultrakinetic anime principles are found in the television series Cowboy Bebop. In fact, the mode is so pervasive, an examination of just the opening credits of the series reveals each factor.

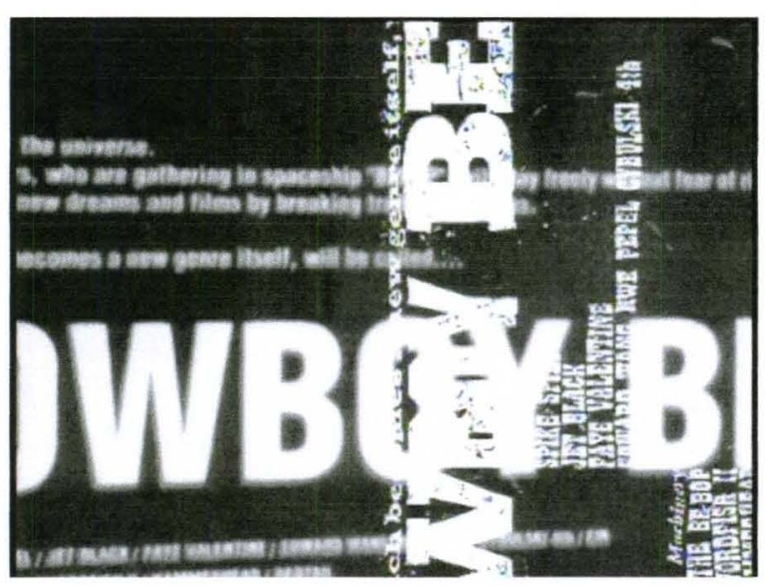

Ultrakinetic written words, density and depth in the use of space:

The initial series title credit layers the phrase "Cowboy Bebop" in both horizontal and vertical directions, accompanied by rapid-fire lists of character names and what appears to be a description of the setting of the series.

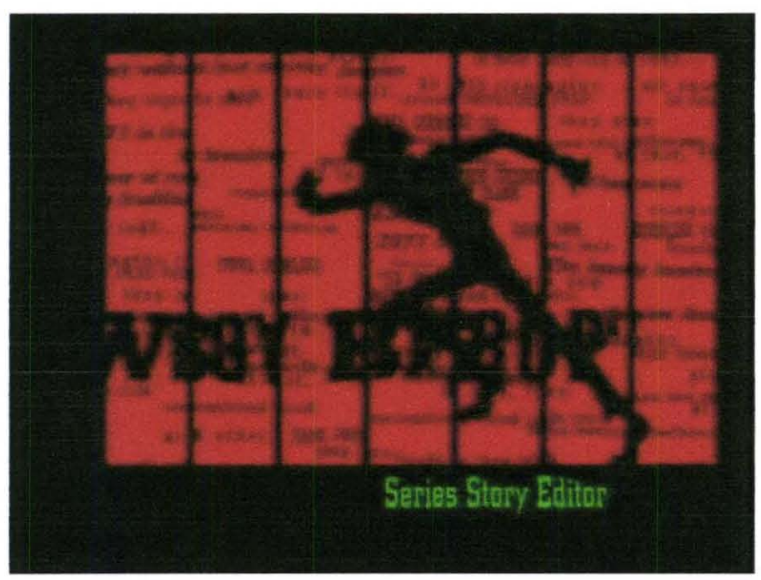

Ultrakinetic color and intensity of movement:

The black silhouette of the main male character, Spike, runs in the opposite direction across text that is scrolling from left to right, but too fast and too blurred to read. 


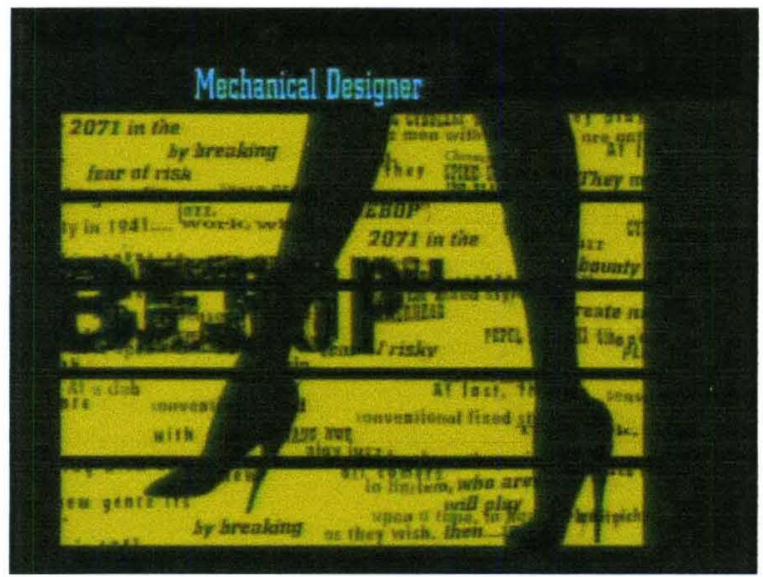

Ultrakinetic slowed motion and written

word:

The story of the Bebop and its inhabitants

begins to come into focus in text behind the slowly strolling high heeled legs of the unlikely heroine, Faye.

Ultrakinetic stillness and simplicity of visual:

\section{Art Director JUNICHI AZUMA}

Spike's solo ship in black outline on a blue background, hovering in place before rocketing forward, leaving a trail of yellow.
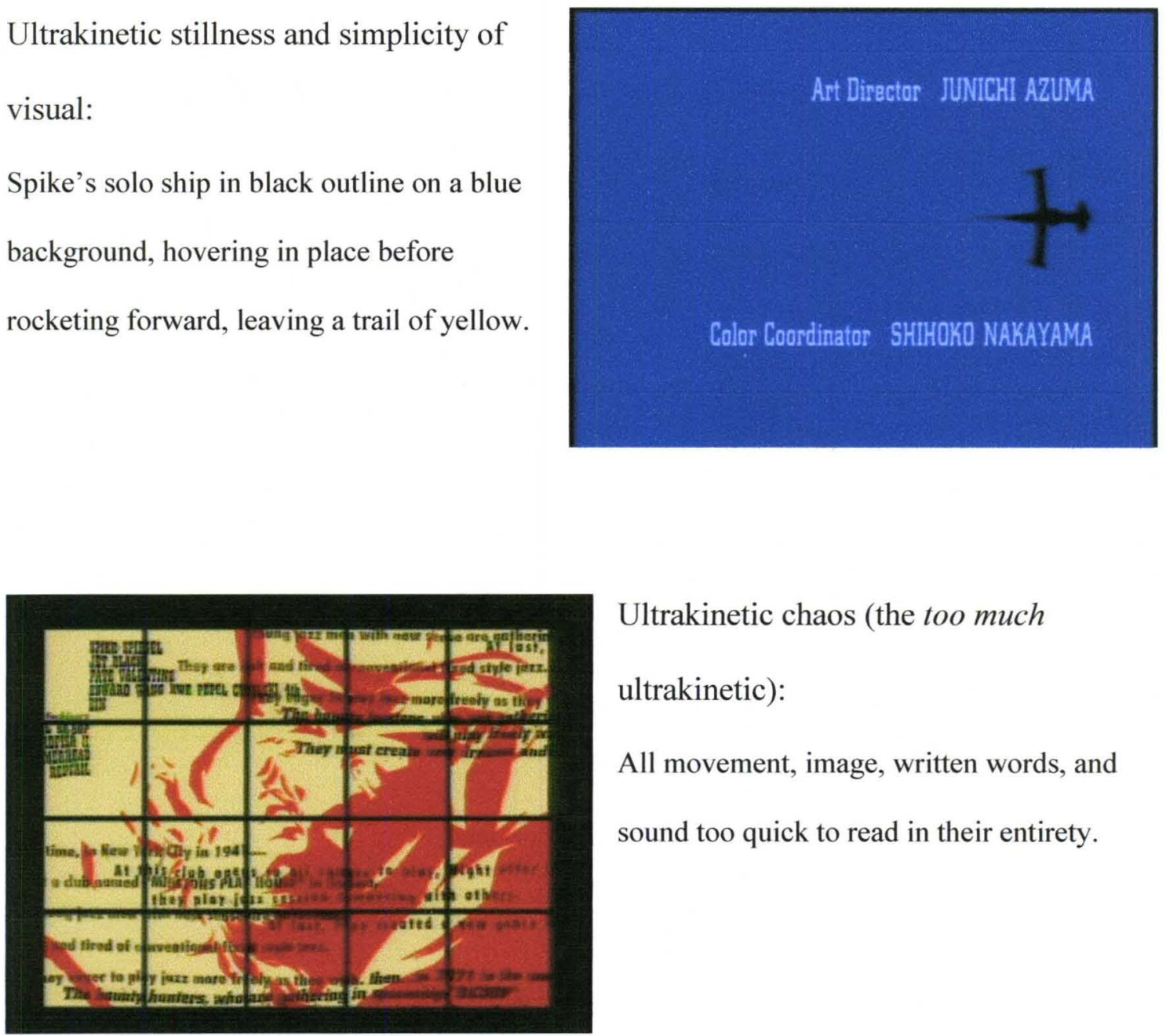

Ultrakinetic chaos (the too much

ultrakinetic):

All movement, image, written words, and sound too quick to read in their entirety. 
Finally, the last two images in the credits each appear with a distinct accompanying beat in the theme song:

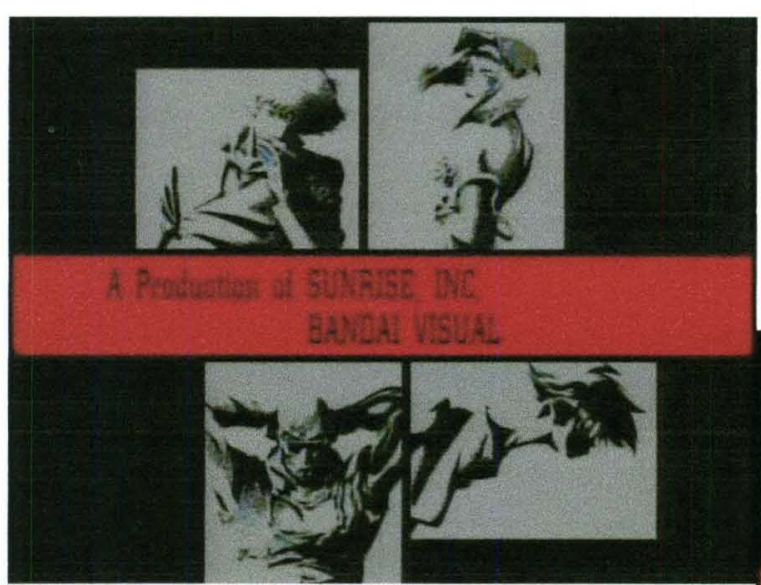

The four main characters, Spike, Faye, Jet, and Edward presented first in stylized drawing, floating across their respective rectangles.

Then, with the final thump of the drums, in dramatic silhouette.

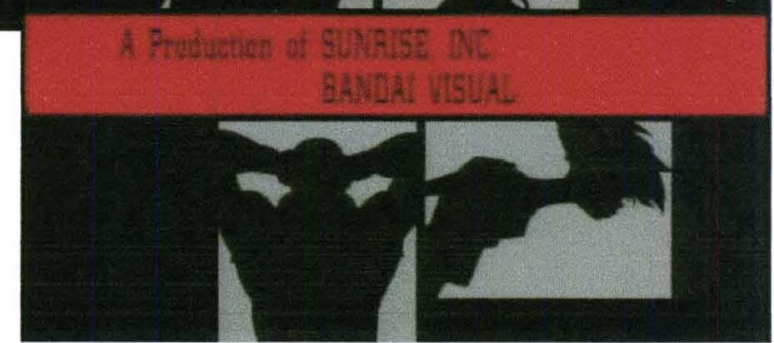

In a visual rhetoric pedagogy that includes ultrakinetic anime, Cowboy Bebop (the series more so than the film) provides a resource for addressing the concept of the ultrakinetic in its entirety.

A few theorists to remember for working with Cowboy Bebop:

- Hess (hip-hop)

- Sirc (punk, hip-hop, rap)

- Schon (jazz)

- Rice (hip-hop and other cool music)

A pedagogy based in ultrakinetic anime does not

exclude necessarily any other methods or materials. Rather, it seeks to provide a specific variety of multimodal text for discussion, analysis and to potentially serve as a model for the breadth of products we may choose to compose. With the growing number of free and share-ware programs for all processes of composing (including image, film and sound editors) becoming available, multimodal products should grow to be expected in 
the composition classroom. In a pedagogy of ultrakinetic anime, students could find great opportunity in an assignment that challenges them to mimic the ultrakinetic style of the Cowboy Bebop credits with the content of their choosing. Or one in which, after viewing (reading) Grave of the Fireflies, they seek out (or create) images that express a perspective of a story that is not the one commonly told or most accessible. In Chapter 4, I explore a few similar applications, including composition work suggested by other ultrakinetic anime outlined in this chapter as well as activities in visual rhetoric that substantiate or would benefit significantly from the use of texts like ultrakinetic anime. Such assignments, I propose, foster the possibility of students functioning as "compositionists-at-large": direct and active participants within their environments, whatever mode(s) information, its transference, or the reaction/dialogue may take. 


\section{Chapter 4}

\section{Toward an Ultrakinetic Visual Rhetoric Pedagogy}

\section{As Schon describes in The Reflective Practitioner, "both ordinary people and professional practitioners often} think about what they are doing, sometimes even while doing it" (50). Here, theory and practice are termed simply as "think" and "do," respectively. This chapter explores different pedagogical "thinking" regarding visual rhetoric and the "doing" activities that result.
The idea that theory and practice not only can, but should be connected resonates with both teachers and learners. A correlation of the academic pursuit of truth or theory and the conception of practical applications seems intuitive. As an instructor of writing, a student of communication, and a human being who observes our rhetoric, I assume the interrelation of what we believe to be true and the actions we take. The reflective practitioner's role also seems an intuitive one in which the cycle of examination of the assumptions we make and comparison to our actions (and vice versa) is perpetual (Phelps). The authors of Writing New Media support their discussion with a reiteration of the need for practical application to be present side-by-side with theory: "Because we [Selfe, Sirc, Wysocki, Johnson-Eilola] believe that practice and

theory clasp like hands, each chapter is followed by classroom and homework activities that grow out of, and in turn, have shaped, the rationale" (vii). One of the aims of this dissertation has been to explore visual rhetoric pedagogy, especially in terms of classroom practices intimated by the study of what I have termed the ultrakinetic anime text. Wysocki's expression of what it means to move toward "generous reading" in 
Writing New Media helps frame an opening for using the ultrakinetic text in the composition classroom:

If we want to achieve abilities to see and hear voices that we traditionally haven't, and to open composition even more to those whose ways with words and pictures don't look like what we know and expect—-then generous approaches to texts that look different and practice in making texts that look different and that therefore position us differently, seem to me worth exploring. (23)

In the contemporary classroom "texts that look different" encompasses many visually rhetorical mediums and electronically mediated composing processes, including frequently used programs like PowerPoint and the Adobe suite for visual computer design: Photoshop, Illustrator, InDesign, Flash and the web editor Dreamweaver. With open source freeware video editing software versions of Movie Maker and Blender (for animation) available, some of the barriers for students to create film and other visual content are disappearing. The demand for students to understand how to read and compose using sources like PowerPoint is nearly ubiquitous. In the academic setting, it is almost assumed that the average class participant will be able to present material in PowerPoint slide format. In the business community, the question is not, "do you have a PowerPoint presentation?, it is more likely, "is your PowerPoint on a flash drive?" A level of sophistication of usage is also assumed, as a bare-bones presentation will not impress. With the current cultural climate, it is necessary for student writers to become true composers, contributors to the multi-literate environment that surrounds us. A visual 
rhetoric pedagogy that includes ultrakinetic texts will be useful for allowing students to practice the reading and production of multimodal visual texts.

In Chapter 3, I spend some time defining the ultrakinetic text, how it functions, and why it is important for consideration. In Literacy in the New Media Age, Kress notes that "in the era of the screen and of multimodality some fundamental changes are inevitable as far as forms, functions and uses of writing are concerned" (61). In this chapter, I investigate a few existing "forms, functions and uses" in writing assignments from the visual rhetoric pedagogy of others as well as suggested exercises of my own that help us "practice in making texts that look different." Sirc, Selfe, and Wysocki each serve as models for the expression of both theory and practice regarding visual texts in the composition classroom. I also look at changes toward more visual and multimodal content in K-12 assignment construction as described by Frey and Fisher. For other models, I look at the overlap of composition methods with the comics suggestions of McCloud. For a foray into the "real" world, the recent vogue practice in business of graphic recording is explored for possible pedagogical application, as is the Post-It note art of Marc Johns. These theorists and practitioners present a model for creating visual texts, a skill that we must begin to articulate with our students while providing impetus and opportunity to participate in a global community whose communication is increasingly multimodal and demands an evolving mulitliteracy.

The New London Group's Multiliteracies includes several chapters that address the issues of "how" this multiliteracy pedagogy can work in actuality and the practicalities of 
the "this" that is working - instructors who are keeping in mind these theories are responding to the current cultural climate and, thus, the needs of their students in a way that teachers who do not incorporate knowledge of and experience with multiple modes do not. Kress offers that he (and others) attempt to "provide ideas and angles with which to supplement what teachers do" (239). I particularly enjoy here the use of the term "angle" to evoke not only that this is a task that possibly requires several points of view and/or attempts, but also the visual nature of working with multiliteracies in mind. This idea of working angles or perspectives is also evident in the way the New London Group defines hybridity: "The term hybridity highlights the mechanisms of creativity and of culture-as-process as particularly salient in contemporary society. People create and innovate by hybridizing, that is by articulating in new ways, established practices and

"The task of the reader of the new page, and of the screens which are its models, is to establish the order through principles of relevance of the reader's making, and to construct meaning from that" (Kress Literacy in the New Media Age 162).

Now, read the above, replacing the word "reader"

with "composer." conventions within and between different modes of meaning" (29-30). Thus hybridity can describe the way a contemporary writer/composer can choose to express his or her argument utilizing not only multiple modes, but significantly through a pastiche of selection of the modes and messages of other writers/composers. Furthermore, as Hocks describes, "hybridity also encourages both authors and audiences to recognize and construct multi-faceted identities as a kind of pleasure" ("Understanding" 632). The activities presented here are all inviting students to engage actively in a way that does generate "pleasure" in the satisfaction of producing a composition that communicates in an experiential way, both for composer and reader/viewer. 


\section{Sirc the Boxer}

The work of the artist Duchamp has been an illustrative example for several of the theorists explored thus far: Berger, Sirc, and McCloud. For each, Duchamp's art seems to serve as an icon of the relationship between the visual and the literate. In his essay "Box Logic," Sirc further draws inspiration from Duchamp as a model for classroom practice. Sirc expresses some frustration regarding the growing complexities surrounding questions of what-to-do in the contemporary composition classroom, particularly what

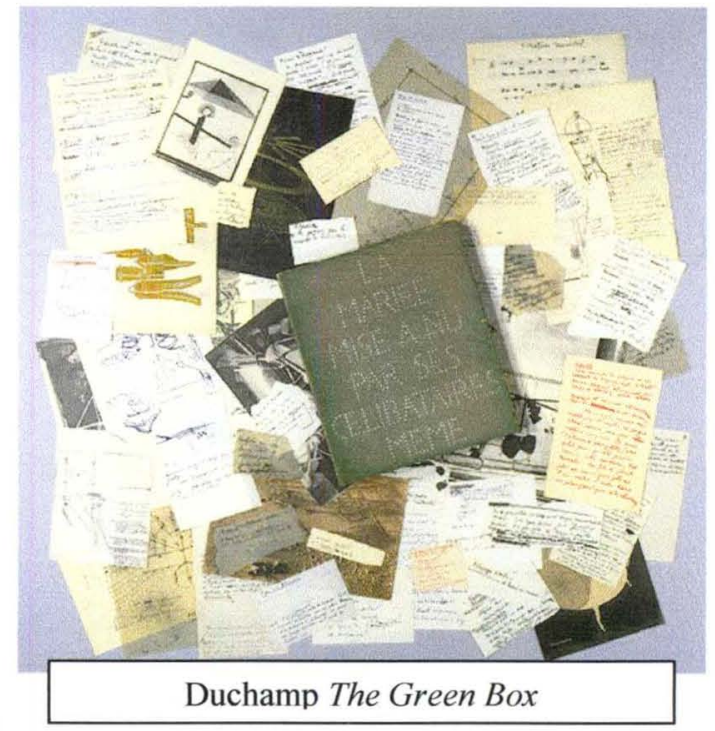
he should do as an instructor: "Well, where I wanted to go, what made the most sense to me personally, was Marcel Duchamp. Specifically, Duchamp's Green Box (1934), the collection of personal notes he made to himself while working on his Large Glass" (111). Duchamp's Green Box is, as Sirc describes, quite literally a pile of slips of paper of various size contained in a labeled green box. Similarly, Duchamp's Box in a Valise collects reproductions of art (Duchamp's own and the work of others) as well as at least one original piece. The added element in Box in a Valise is that some of the reproductions are three-dimensional. For Sirc, the Green Box is important for the example it provides of "text as a collection of interesting, powerful statements" (112). The "box" also provides an allusion to composer as collector, a metaphor that Sirc is 
interested in particularly for research projects. The reading, analysis, selection and synthesis involved in writing seem similar to the activities of collection represented by Duchamp's boxes. A collector is creating a composition that tells its own story: objects and/or other various materials that express a message, something that shows the individual's preferences and communicates both affinity and experience. The writer/composer collects as well: ideas, information, argument, evidence, word, image, sound. Box composition invites the writer to partake in the development of ideas and

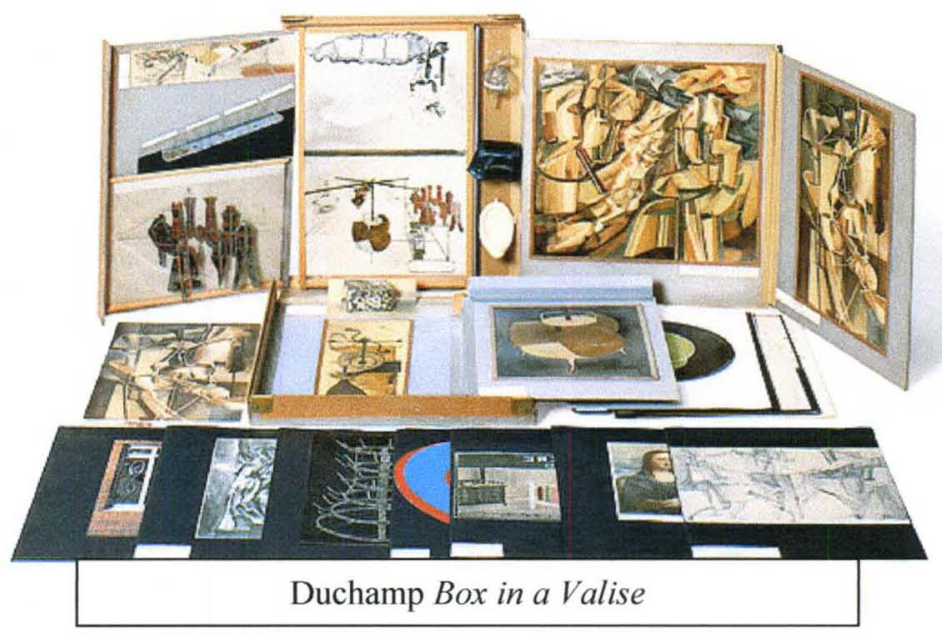
content as a collector does, mediating the information in selection, juxtaposition and labeling or naming. Box composition is also naturally multimodal, creating opportunity for the use of image, sound, word, and texture.

Sirc sees the box as having potential to help the writer/composer (collector) focus on not only a specific message, but essentially the emotional or visceral connection that drew the writer to his or her subject in the first place: "The grammar of the box can keep us grounded in the basic image, in things we really care about" (119). For Sirc, it is important for the writer/composer to be passionate about the work he or she is doing, that such passion is essential to not just effective composing processes, but also for the larger 
scope of learning and communication in general. In his discussion of the writing of his student Greg White, he highlights the raw communicative ability of emails Greg writes regarding his feelings about the music of a particular rap artist. In many ways, Sirc helps

Greg collect his thoughts and express

himself creatively by providing an

alternative space in the writing classroom

for all kinds of communication, from

error-ridden but passionate email to box

compositions.

Sirc discusses how collage is a viable form

of expression for students, one in which

the decisions of selection, placement and

juxtaposition are important storytelling

and compositional elements to practice.

For me, collage has a natural connection to

the concept of the ultrakinetic text,

especially since ultrakinetic can mean that

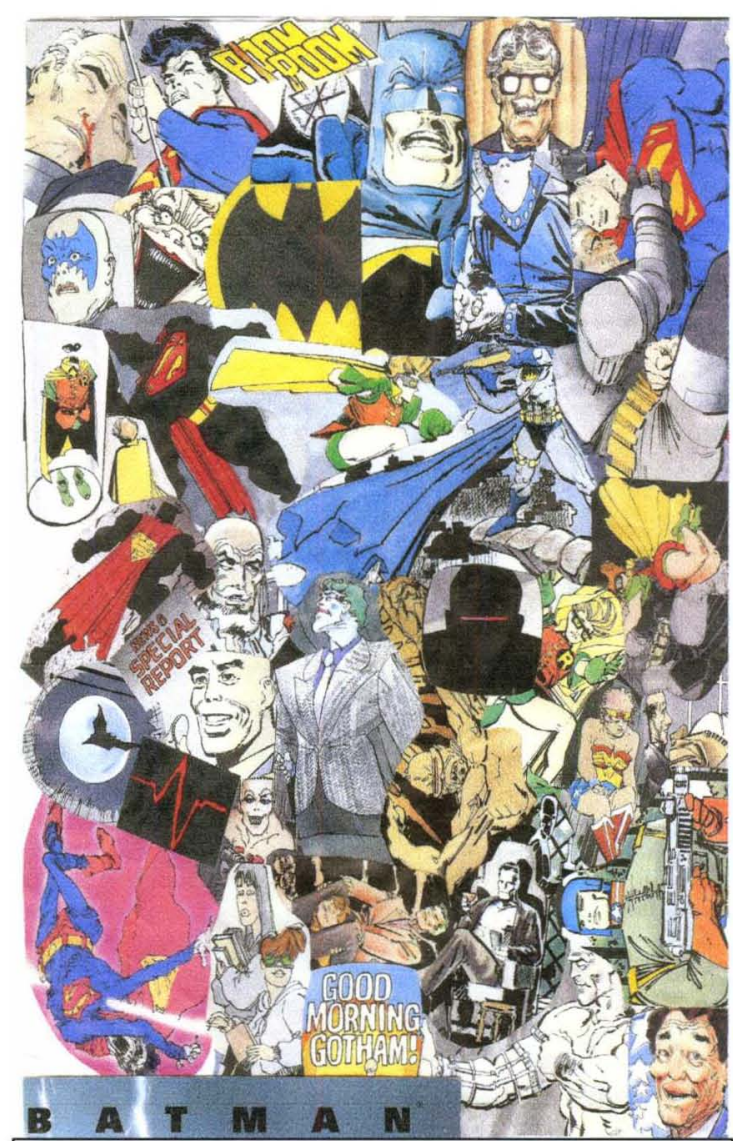

A Kind of Box:

Collage from a first year college writing composer in a theme course on Batman: all images from the graphic novel The Dark Knight Returns. In essence, the entire visual story is now told in this one page.

there is a sense of being overwhelmed by certain visuals, that there is too-much-to-look-

at. Collages, indeed, must be read; they do not present a single image that can be

absorbed immediately. Berger argues that some images, in his argument specifically

larger oil paintings of certain styles, function in this manner as well. We only are able to look at part of an image at a time, he contends. This may be true, but Sirc's "Box Logic" 
intimates that the parts are not only what makes the whole, but that a more fragmented presentation can capture a writer/composer's process and fundamental message: "The box as dossier allows a credible collection of the variety of field-notes my students amass on their journey" (123). Thus the "variety of field notes" or the parts of the whole, the bits that chronicle the student process, are viable material for consideration. What makes a box (metaphorical or material) "credible" is the collection: the composition. This hearkens to how Yancey describes the portfolio process as requiring the writer to practice "selection, collection, and reflection." A portfolio, by Sirc's description of "box logic," could be viewed as a type or form of box composition.

Sirc provides an example of the application of his "box logic" in the

Berger explains how we can read an ultrakinetic text.

Sirc shows us how students can compose ultrakinetic texts. activity "A Basic Box": "Students juxtapose quotes and images...

Once students have selected and arranged their juxtaposition. They can write their own expressive commentary, reflection on what the juxtaposed texts mean to them... and artfully integrate it into the work" (131). (This is similar to the reflective essay in the AntiEssay assignment, subsequently described in this chapter.) Because

working in multiple modes in the classroom environment may feel foreign to many students, Sirc suggests that the risk-taking of completing and presenting the project should be rewarded by the instructor, who should "be very encouraging with grading" and use successful execution, even in small steps and gestures, as building to more complex acts of communication (133). For example, Sirc suggests that assignments like "A Basic Box" can scaffold toward other efforts, such as purposeful web page design. 
The "Basic Box" could serve as an apt initial assignment when working with an

ultrakinetic text, by asking students to center their

image and quote selection on the ultrakinetic text.

In Sirc's box composition, the box and its contents

can assume many forms, at the discretion of the

composer, but the overall result is described as more

effective than other forms:

It's the box-artist's goal: text rubbing against

text, making an arrangement of materials to

see what could be done with them. The open-

ended forms and available materials permit an

intimacy and intensity that more mediated

genres make difficult; students see writing

elementally, as a material encounter rather

than commodified production. (146)

The "arrangement of materials" is the work of the

composer/collector. I take "mediated genres" to

mean more traditional forms of writing

communication in which the format is dictatorial and

confining. One of Sirc's goals is to shake up our

Experimenting with Basic Visual Rhetoric: Color Wheel Sentence Diagramming

In an early classroom teaching experience, I was faced with a fairly prescriptive set of requirements for a special section of high school English for juniors and seniors who were termed at-risk. Some of the students were struggling with sentence structure in a unit that utilized traditional sentence diagramming. Many of the students seemed to respond to projects that had a more visual nature. In recognition for this learning style affinity, an artistic approach was applied to the sentence diagramming. We reviewed basic color theory using a simple color wheel composed of primary and secondary sets of colors. Subjects were designated primary shade red and predicates, blue. The dividing mark between was yellow. Thus, knowing from the color wheel that red and yellow combine to form the secondary color orange, subject modifiers were drawn in orange. As yellow and blue combine to green, predicate modifiers were drawn in green. Students were provided their choice crayons, markers, colored pencils and paints to accomplish the technique.

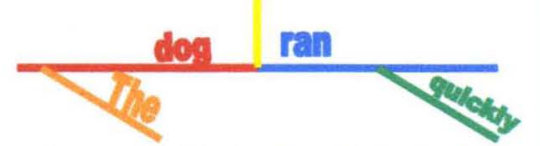

This method helped students make interdisciplinary and multimodal connections while providing a mnemonic for grammar and a sense of fun in the work.

expectations of students, the classroom, and ourselves as instructors by inviting us to

think about material considerations when collecting and ultimately producing text. He is 
hopeful about what a reconsideration of modes and methods will do for each of us, the growth of our understanding, and our abilities to communicate:

With the essay displaced, our new classroom genre might best be called a diary journal repository laboratory, picture gallery, museum, sanctuary, observatory, key...inviting us to see things in a light in which we do not know them, but which turns out to be almost that one in which we have always hoped one day to see them bathed. (146)

With the introduction of box composition and source material like the ultrakinetic anime discussed in Chapter 3 of this dissertation, this new classroom genre seems promising for helping to address the complexities of a multiliterate culture. This is important for a $21^{\text {st }}$ century mentality in which the literacy expectations for college-educated students have changed. A visual rhetoric pedagogy of ultrakinetic texts provides a more immersive, experiential education in current communication forms.

\section{Making Room for Self(e)}

In her presentation at the 2006 Watson Conference, Selfe cautioned that "when we focus on only one modality, we make our lessons less accessible" and that there is a danger that we will "limit students' rhetorical agency to our own interests." This does not mean that a responsible instructor is versed in every mode and interest possible; it means that a responsible instructor is open to a variety of modes and interests. This involves a certain willingness to take the risk to become the instructed in the classroom. "This activity depends on teachers being willing to become students," Selfe advises in an assignment 
description in "Students Who Teach

Us" in Writing New Media. The

activities she provides also ask

students to commit to the risk-taking

of teaching and learning from one

another, in the spirit of Freire's

suggestion that teachers "must be

partners of the students in their

relations with them" (75) Freire's

selection of the word partner

emphasizes the reciprocal nature of

teaching and learning, found also in

Selfe's exposition. The individual

experience is instructive no matter if

that individual happens to be teacher

or student. For many of the activities

she describes, Selfe also includes

sample response sheets that help guide

both reaction and interaction with

developing compositions. Like Sirc,

there is a box, metaphorical or

otherwise, to provide some direction:

the ribbon weight anchor to the
In Writing New Media, Selfe introduces "Technological Literacy Autobiographies" as a means of situating student experience in a meaningful and descriptive way (59).

My first encounter with the concept of a Literacy Autobiography was in Huot's Teaching College Composition course as a potential writing exercise suitable for our first year 101 students. Literacy can be conceived in many different incarnations; an autobiography is the story of one's self. Add these elements together and voila! a Literacy Autobiography. The student's interpretation of the term literacy proves revelatory as to some of his/her analytical thought processes. The sharing of self in the autobiographical form also gives the instructor an opportunity to glimpse into the thoughtful mind/life of the student. Thus, I particularly enjoy the "Lit Bio" as an introductory exercise-a way into the classroom relationship. If the student's sense of literacy is simply I-know-how-to-read, the initial description of literacy meaning many things is an immediate challenge. By extrapolating the base understanding of literacy to a realm where many other associations are appropriate, the literacy autobiography can include memories about words, sounds, utterances, reading, writing, storytelling, moments of understanding and times of miscommunication.

In the most recent Teaching of Writing (Eng 540) course that I taught, I quickly explained that one version of my own literacy autobiography might include a reflection on how I believe I have wandered my way to this point - a teacher, a student of rhetoric, and a business professional whose job it is to consider carefully the weight and possible perception of every word and image in a public document. I mused for my students, seeking to be a model for the exercise. I described how my family is from a mostly comfortable but relatively modest background. I did not struggle without necessities, nor did I have everything - certainly not always the latest or newest toy or tennis shoe (different objects of desire for different ages). But my mother had a firm policy that a book would always be made available to me whenever I wanted. Sometimes a bookstore trip for something to keep; or, more often, a library trip. I fondly remember bringing a completed list to the librarian one summer. Remember those summer contests? Prizes for reading a certain number of books... The librarian was incredulous at my timeline; she did not believe I had read that number of books in that number of days. I showed her how carefully I had recorded each author and book title. My mother informed the librarian that I had indeed read every word of every book listed and fully deserved whatever bookmark or tote bag should be awarded. This is the moment that is cemented in my memory-my mother practically stamped her foot and indignantly demanded "Quiz her! You can quiz her on any book. Ask her any question you want." I was so proud. Proud that my mother would defend me in this way, proud that I was such a quick reader, proud that I knew what was in those books. I could tell other stories, I let my students know, but this one seemed the most important to me somehow. I further delineated for the class that in a text form I would likely spend some time reflecting on why the story felt important: what it seemed to represent, feelings it evoked, implications.

A combined Selfe/Sirc assignment would ask students to write their Literacy Autobiography in the form of a box composition. With the addition of images of my younger self, my mother, a pop-up book, prize bookmarks and gold star stickers, my narrative is transformed into an ultrakinetic text to be experienced by an active reader/viewer. 
balloon of our ideas. The worksheets include reminders regarding assignment objectives as well as task steps to follow. Thus, there is a framework to help guide and focus the creative production of the composition. Some sheets are for the "composer/designer" while others are completed by the "reviewer" or "reader/viewer." Selfe explains her choice of language in these designations:

I will use the term composer/designer, instead of "author" or "artist," for instance, to describe an individual who produces or creates a visual text....to refer to the reader of visual texts, I will use the term reader/viewer and, for the complex set of activities associated with understanding and interpreting a visual text, I will use reading/viewing...they are suggestive of the richness of visual compositions. (69)

I find these delineations to be useful in encompassing the more complicated nature of portraying the breadth of processes involved in multimodal participation, for example the viewing/reading of ultrakinetic texts. The writer doesn't just write anymore and reading is not just taking in words on a page. Selfe's examples provide not just space for but a genuine invitation to participate in multimodal critique and production. In the spirit of this challenge, I offer an example endeavor in visual literacy of my own: the Anti-Essay.

\section{The Anti-Essay}

Jeff Rice criticizes the language used in one of the assignments in McQuade and McQuade's Seeing and Writing: " 'write an essay' is the prescriptive...One might wonder the purpose of reading an advertisement at all. Why don't these authors ask 
students to write their own set of images?" (152). Asking students to author texts that significantly use or include visual elements is the core of the writing activity I call the "Anti-Essay." For me, the genesis of the Anti-Essay lies in working with Berger's Ways of Seeing. In 1972, Berger remarked: "In no other form of society in history has there been such a concentration of images, such a density of visual messages" (129). The first time I read those words, it was 1993: I thought, wow, what about the "density of visual messages" twenty years later? In 1998, my first year teaching writing in college, I wondered, what will students think of these words, five years later? Now, it seems that the sheer volume of images we see is at times overwhelming. The more "dated" the Berger text becomes, the more pertinent its focus Impromptu Anti-Essay (performance version):

"I thought one of the best instances of collaboration, true Composition as a Happening, was when four students dropped peer discussion and, each taking a turn, did a 20-line rap song on the subject of Mother Teresa" (Sirc, English Composition as a Happening 199). on visual communication. Based on a BBC television series, Ways of Seeing is imageheavy itself, with several of the essays containing only visuals. Despite how accustomed we are socially and culturally to a daily overdose of visual stimuli, being confronted with an "essay" that doesn't include verbal text is still surprising to some students, even in 2009. This experience of newness creates an opportunity for an awareness-in-reading that typically produces some interesting analytical results. I also wanted this sense of working through and within the text in an attentive and responsive manner to extend to the text production inspired by the reading as well. In those first teaching endeavors, I asked students to respond either directly or indirectly to Ways of Seeing by producing what I termed an "anti-essay." The basic instructions for the assignment were to provide an argument: articulate, well-supported and evocative, but without a traditional text-only 
essay. The only way

to perform this

activity incorrectly

would be to submit a

typical MLA or APA

formatted word-only

text. I invited

students to play with

images as their text,

combine text and

image, or re-imagine

verbal text in a more

visual categorization.

Other modes were

welcome as well:

music, for example,

or film.

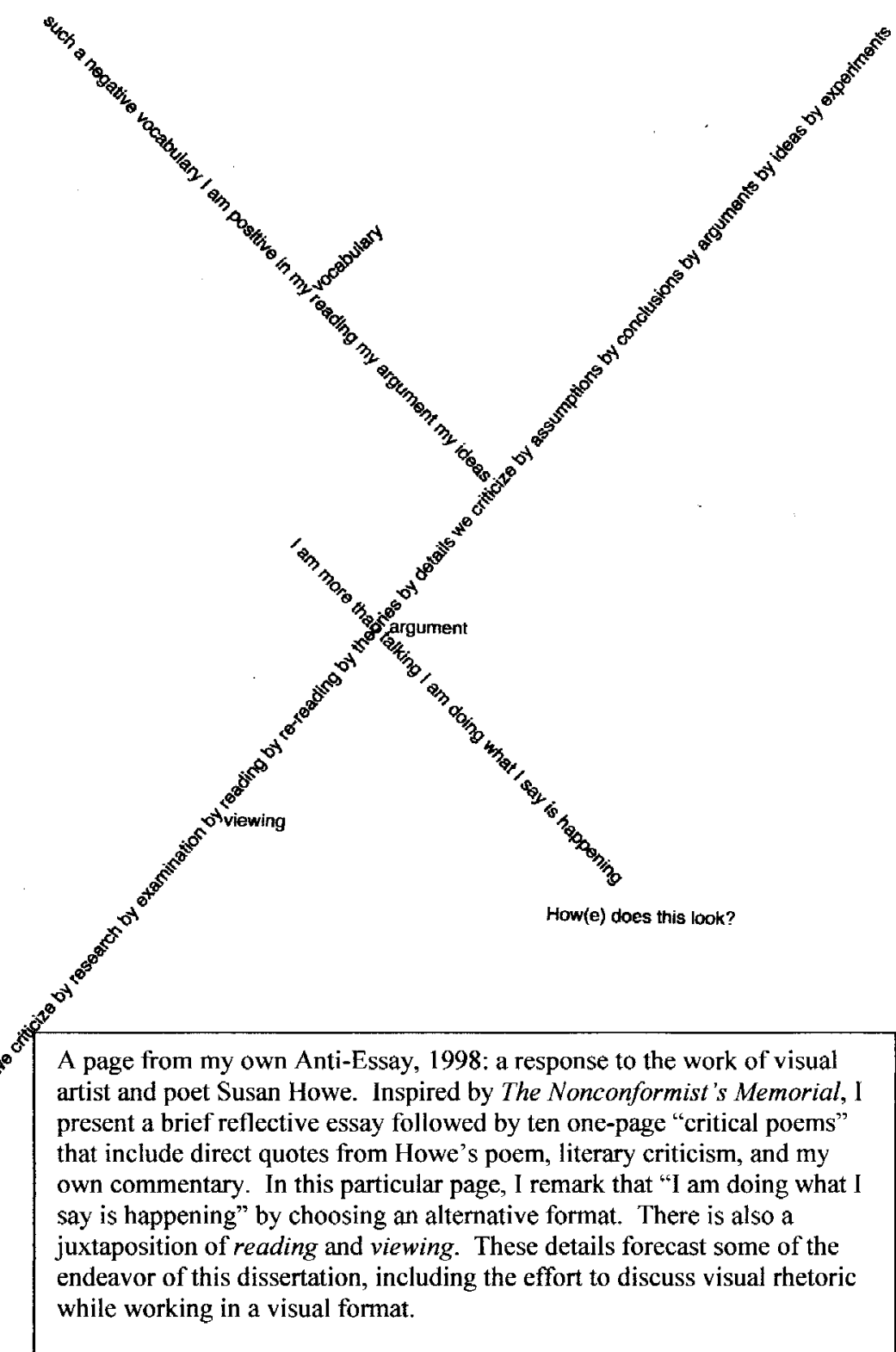

The opportunity to respond to a text that seemed new and unusual (Ways of Seeing) in a format that was potentially equally unique seemed to create a sort of academic symmetry that produced thoughtful, well-wrought compositions. When reading multiple modes, it may be natural to interact in-kind in a multimodal fashion. When students work with ultrakinetic anime texts (the multimodality of choice for this exploration), a method like 
the Anti-Essay may allow for a more complete expression of their arguments. In regard to providing opportunities to analyze and produce designed texts, Hocks provides justification that "this approach to pedagogy asks teachers not only to incorporate new kinds of texts into our classrooms but new kinds of multimodal compositional processes that ask students to envision and create something that perhaps does not yet exist" ("Understanding" 645). The results of challenging students in this manner can be very rewarding.
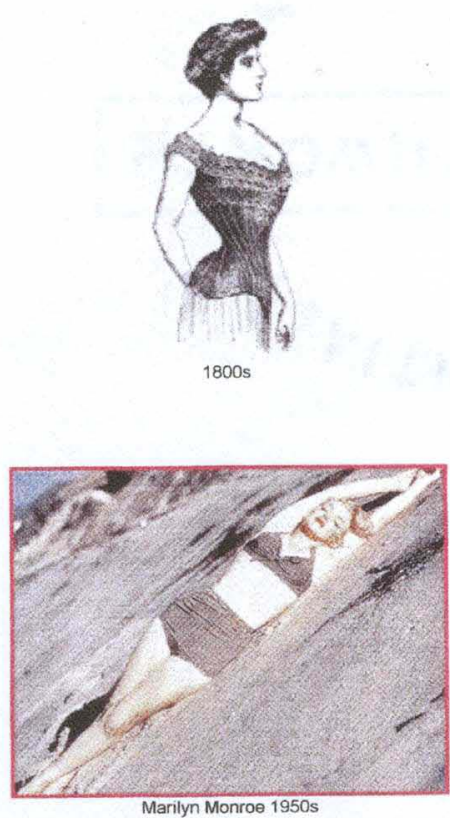
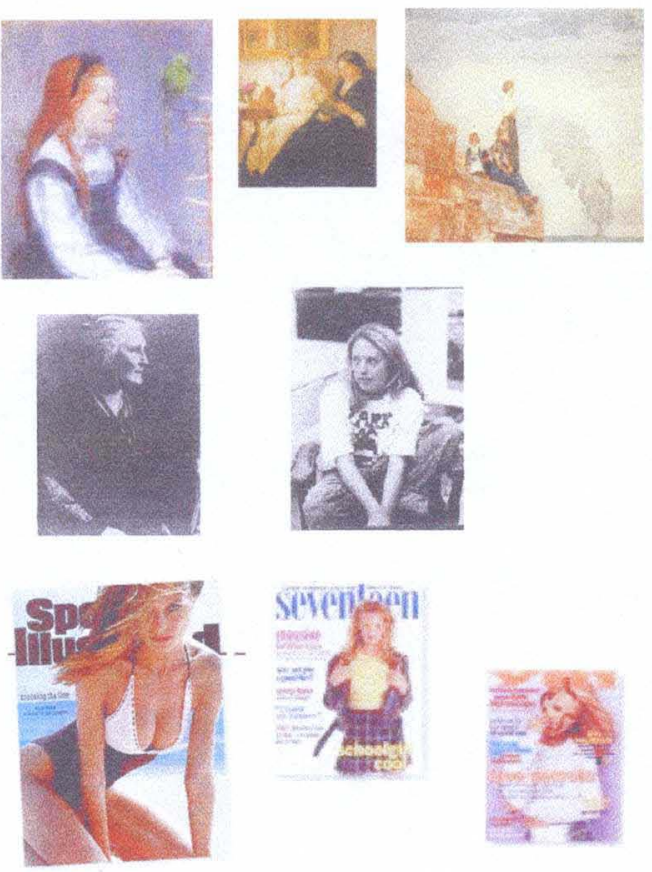

These two sample pages are from different student essays that are directly responding to Berger's pictorial essay on images of women. In the first example the writer/composer choose to present all of her images two-to-a-page in this manner. The effect draws each pair of images into direct comparison. In the second example, multiple images are 
juxtaposed, but the writer/composer did not explore the rhetorical possibilities of overlapping collage (a technique the Berger essays display).

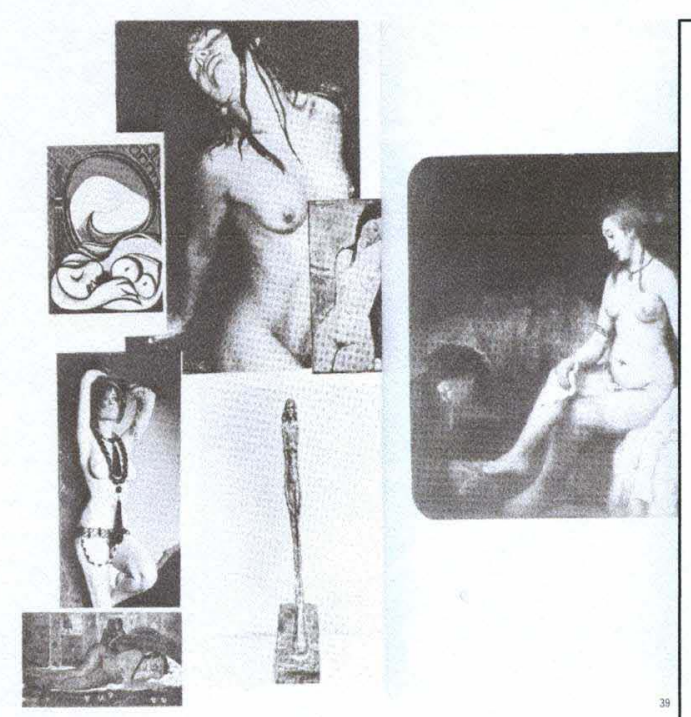

Berger's pictorial essay on women features a section on the perceived difference between pornography and art, the nude vs. the naked (39). Students have remarked that a similar argument presented in another format, like text-only, would not have captured their attention to the same degree. One student admitted that she would have argued vehemently that a pornographic photograph and a work of fine art could not be comparable, but that this single page called her to re-evaluate and re-articulate the terms in which she was describing her thoughts.

Students also reveal the impetus to respond in-kind to the text; the visual nature of the presentation evoked a desire to present an analogous visual argument.

Students do not allow (nor should they) the subject matter of the Berger examples to limit their choices. In this example, a student wanted to research provocative images of slavery in order to express his own emotional response to a slavery narrative he was reading for another class. In his reflection regarding the assignment, he indicated that

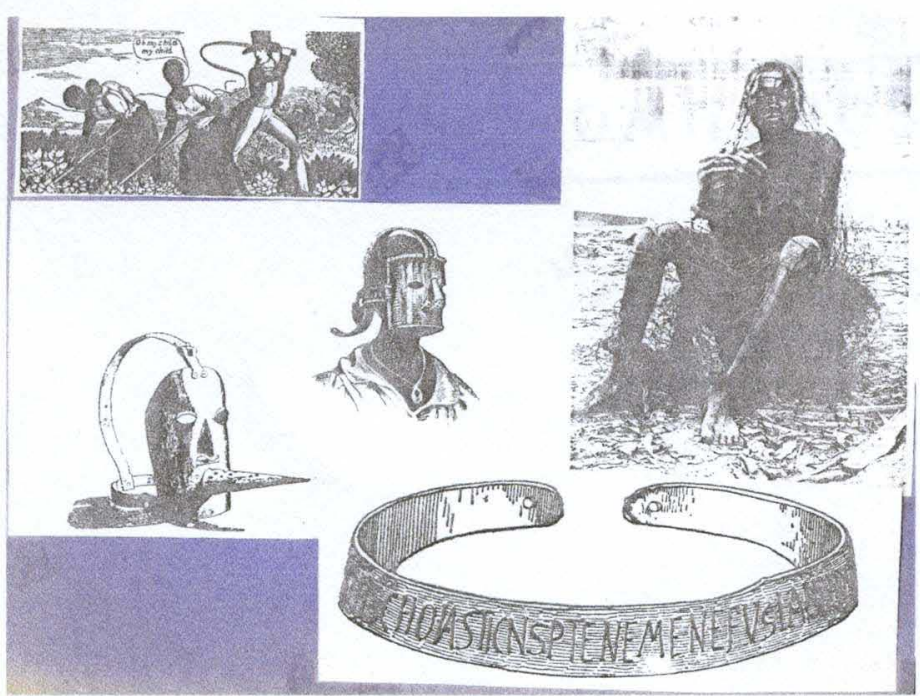
something about manipulating the physical copies of the images (cutting them out, affixing with glue on sheets of construction paper) felt like it brought him closer to his subject, as if he had more control over the presentation of the images than 
he would the verbal text of a more traditionally written essay. He also discussed the care he took in his design, even to the selection of color for the background papers (dark colors for a dark subject). This student reflection helps capture part of the intended consequences of the Anti-Essay assignment: students creating a format for their expression that is closer to themselves and their subjects. Hocks has written that "design projects require writers to look at successful models, to think deeply about audience, to design visual and verbal arguments together, and to actively construct new knowledge ("Understanding" 651-52). When the use of multiple modes is introduced to the student composer, a particular kind of agency develops, one in which the student has new levels and means of controlling his or her composition. The reflective essay provides another component that stimulates expression by asking the student to provide self-analysis regarding the composing process and the overall experience of composing in a new way. In a pedagogy of ultrakinetic texts, reflection becomes an essential part of the interactive experience of reading and responding to intricate multimodal content. Ultrakinetic helps describe visuals that, due to the highlighted intensity of their nature, incite a reaction in the viewer/reader. Just as reading an ultrakinetic text is an experience, so too is the creation of one.

The Anti-Essay provides some interesting opportunities in the interaction of collaborative writing as well. An Anti-Essay on the subject of gun control is the result of a collaborative writing assignment in which the students were asked to select together all aspects of the composition, from subject matter to final product. This particular essay definitely functions within Sirc's Duchamp-inspired box concept: text on text, layered 
voices to be considered in

whole and in part. The

essay includes a separate

section of research

description and a reflective

memo, but the "main"

essay consists of personal

narratives on paper, cut and

arranged to form one

comprehensive expression.

In their reflection, the

group members are

transparent about many of

their choices. They chose

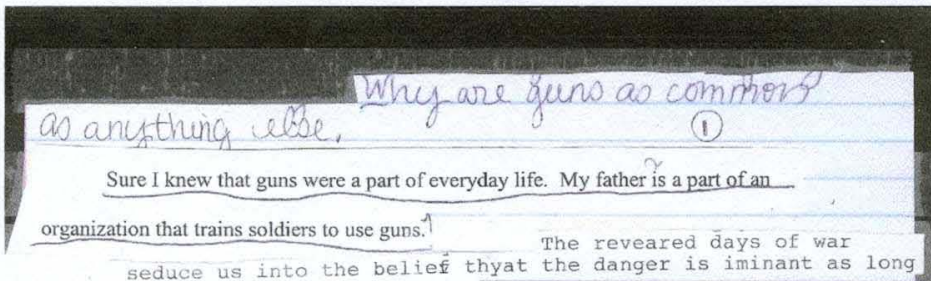
as talk the wepon is absent.

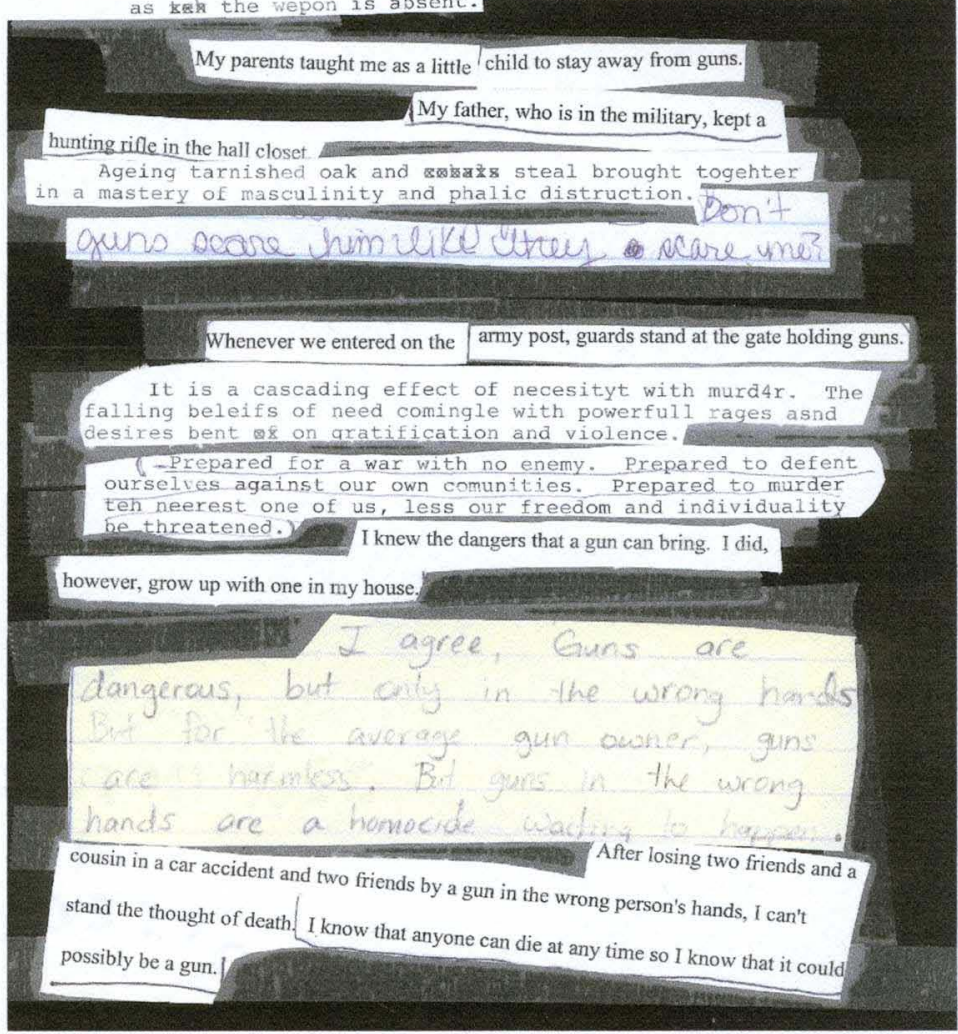

not to edit for error in any

of the text in order to present the "typos of real people," a sign of their desire for

authenticity. The handwriting and font types visually display the different voices present

in the text. Furthermore, the reflection indicates that if the essay were videotaped or

audio-recorded, it would be important for different people to read each part. The group

admits they could have chosen another mode, but wanted to have a physically tangible

product that could be held in one's hands and had the "authority of a book." This is

meant to be complicated immediately by the use of visible plastic tape to attach the 
pieces of essay to the spiral-bound thick pages. For the group, this "imperfect" visual symbolizes the raw, emotional nature of their subject matter.

I use the Anti-Essay in conjunction with viewing Tezuka's Metropolis. In this instance, the assignment requires the use of or reference to music as part of the response to the film. As previously discussed in

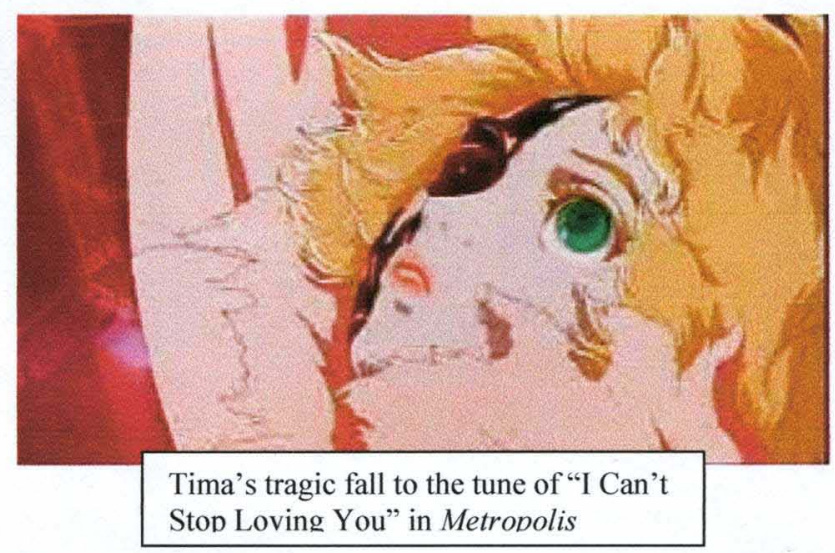

Chapter 3 , the closing sequences of the anime are intensified by the idiosyncratic choice of music. Several students have chosen to re-score the soundtrack by offering new selections of songs to accompany important moments in the film, explaining the significance of their choices and discussing the emotional effect of the original compared to their revision. Another version of Anti-Essay submits theme songs for each character as a way to explore and articulate individual motivations as presented in the film. Cowboy Bebop (both the television series and the stand-alone film) could also benefit from a similar treatment, due to the emphasis in the anime on music as a contributing factor to mood, character development, setting, and tone. A music inclusive Anti-Essay participates in the fun-factor in working with ultrakinetic texts as it encourages the use of a mode many students are passionate about and usually enjoy sharing with one another. The music also serves as a familiar anchor for many students for whom the foray into visual and/or multimodal composition may seem daunting. 


\section{Arranging Shapes on a Rectangle: Bang!}

Another way to anchor the practice of the Anti-Essay is to utilize approachable,

illustrative models. In the Preface to Picture This: Perception and Composition, Arnheim summarizes the work as providing a distinct and accessible example of working in visual rhetoric:

These simple shapes, animated by Molly Bang, do more than tell a story: they offer an order, a kind of grammar for the eyes, a recipe for yet further things to say. Therefore they also teach. Without preaching theory, they point painlessly to principles that can stimulate and guide anybody in his or her work, be he or she amateur or artist, child or adult. (x)

In Bang's own words, her project "describes picture structure in terms of our feelings" (xi). Like Berger's Ways of Seeing, Bang's Picture This is a potential inspiration for student composition of visual texts in the spirit of the Anti-Essay.

Bang's experience as a writer/composer is primarily in the area of children's book illustration, a genre where juxtaposition of word and image is almost standard practice. In writing Picture This, she sought to explain some of the decisions she made intuitively as a composer, like the reflective memo portion of the Anti-Essay. The text begins with Bang rendering her process in creating a specific, simple story image: Red Riding Hood in the woods with the Big Bad Wolf. Each step is deconstructed, from shape selection to size, color, placement and other details. For example, she looks at the consequences of color selection of the wolf in conjunction with the already defined red triangle of Red Riding Hood. 
On the left she finds the lavender wolf "a bit disturbing" where the black wolf on the right provides "cohesion" to
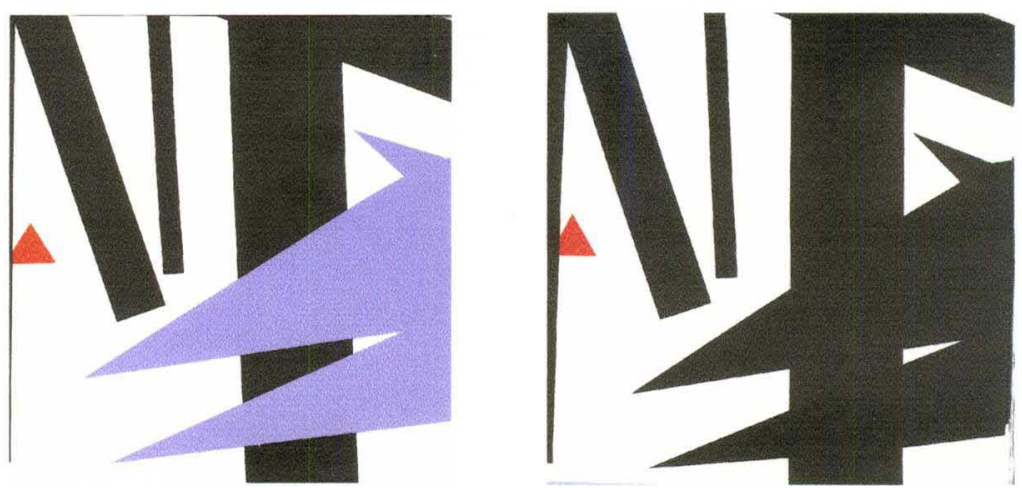

the overall picture (34). For Bang, understanding and the ability to create are joined:

"Our feelings arise because we see pictures as extensions of our real world. Pictures that affect us strongly used structural principles based on the way we have to react in the real world in order to survive. As soon as you understand these principles, you will understand why pictures have such specific emotional effects. You will understand how pictures work." (54).

Bang presents ten basic visual rhetoric guidelines ("principles") that are relatively simple for students and visual rhetoric pedagogy practitioners alike, such as "diagonal shapes are dynamic because they imply motion or tension" (62) and "the larger an object is in a picture, the stronger it feels" (100). These principles provide a vocabulary and concrete examples for comparison for viewer/readers to reference when describing what they experience in an ultrakinetic text.

After working through examples of these principles, Bang suggests an activity involving "arranging shapes on a rectangle" as an opportunity for her audience to practice the ideas 
(135). She comments on her recommendation to use pieces of construction paper and scissors only for the composition:

First, construction paper is cheap, and it is familiar. It is easy to work with and eminently 'nonthreatening.' For many of us, cutting construction paper puts us back into nursery school or kindergarten again - into a time when we got absorbed in the task at hand, with little thought of anything beyond the Right-Now-of-Making-This...Second, working with cut paper encourages experimentation. Every piece of paper can be recut, repositioned, or replaced with a different piece or pieces...Playing with paper is making a series of beautiful 'controlled' experiments.

As described, this method is incredibly easy to replicate in any classroom. It is also a simple matter to alter the assignment to a technologically mediated one, perhaps by creating the shapes in a design or painting program.

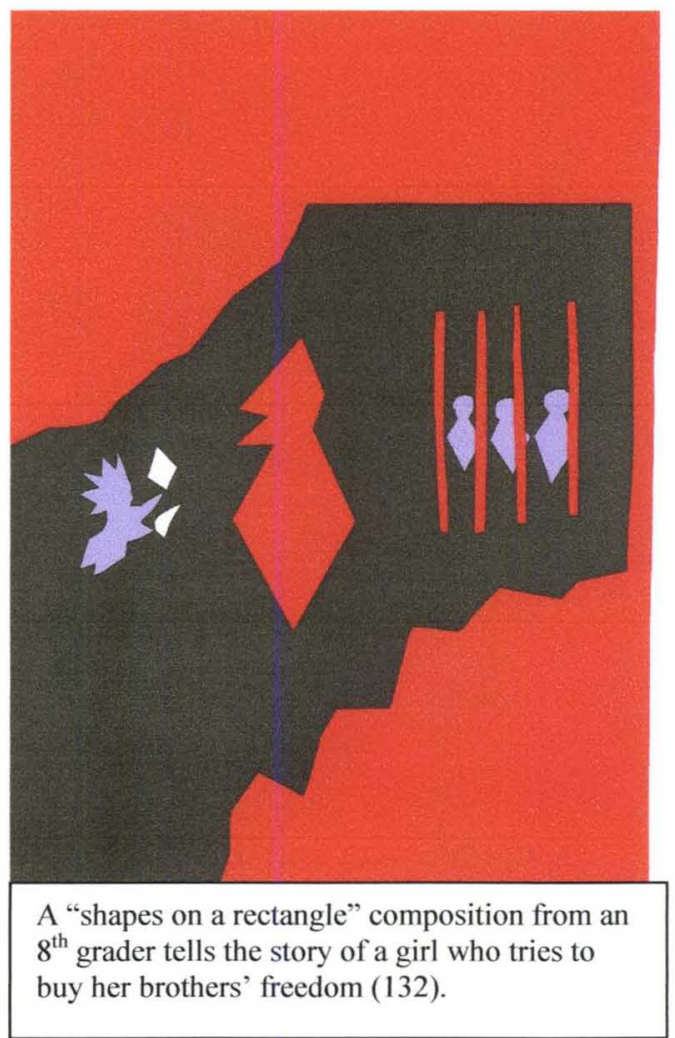

One focus suggested by Bang is applicable to working with ultrakinetic anime texts in a more obvious way: "Illustrate a cover for a book or a poster for a movie. (Ask yourself the same questions as for the other pictures, but add a further question: How can I most 
effectively use the letters to enhance the feeling I want to evoke? Look at how words are used on a variety of posters and book covers.)" (140). The questions she refers to are those she provides for consideration when beginning any visual, literate or combined composition and its intended impact:

1. What is the essence of the person/creature/thing I want to represent? What specific elements in this situation evoke strong feelings in me? How can I accentuate these?

2. What feeling do I want to evoke with this picture, and what principles might I use to do this?" (137).

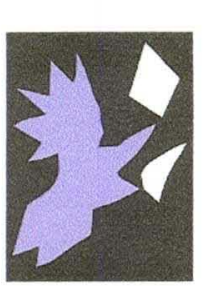

With Bang's emphasis on function of design being uniquely positioned to engender emotional audience response, it seems a natural to consider "Arranging Shapes on a Rectangle" or a variation thereof for work with ultrakinetic texts like Grave of the Fireflies in which the emotional content is ready to be un-packed. The same way dynamism is present in the ultrakinetic stillness of Grave of the Fireflies, the principles evidenced and practiced in "Arranging Shapes on a Rectangle" call attention to the expressiveness of single moments and movements.

Bang intimates the larger implications of the principles she identifies and the potential for a variety of composing methods. She does, however, seem to believe that beginning with her fundamental technique inherently will lead to sound design thereafter: "Playing with cut paper forces us to concentrate on the basic structure and on how this structure affects our emotions. But this is the basis for all our visual art forms, the foundation upon which the rest is built. Once you understand how these principles work with paper, you can use 
them in any medium" (141). This reminds me of Sirc's desire for students to be "obsessed" or passionate about their subjects; and how he is excited by box composition because it keep the writer/composer "grounded in the basic image," by which he seems to mean focused on the main evocative idea. "Arranging Shapes on a Rectangle" is a welldevised option for grounding an Anti-Essay within a visual rhetoric pedagogy.

\section{Storyboarding}

Frey and Fisher work together on the opportunities in and importance of considering contemporary visual rhetoric texts for inclusion in the K-12 classroom. Like Bang, Frey and Fisher see comprehension or understanding and visual rhetoric as inextricably linked to the production of effective communication:

We think of visual literacy as describing the complex act of meaning making using still or moving images. As with reading comprehension, visually literate learners are able to make connections, determine importance, synthesize information, evaluate, and critique. Further, these visual literacies are interwoven with textual ones, so that their interaction forms the basis for a more complete understanding. (Teaching Visual

\section{Literacy 1)}

One of the resources Frey and Fisher propose for inclusion is anime. In Teaching Visual Literacy, Chandler-Alcott authors a chapter to further substantiate the claim that the use of anime texts in the classroom is crucial for a $21^{\text {st }}$ century literate society: "more American young people have begun to identify themselves as anime fans. Consequently, literacy teachers can no longer afford to see anime as the province of a few hobbyists 
seeking to position themselves as 'alternative' through appreciation of Far Eastern Culture" (65-66). Chandler-Alcott situates her ideas in relation to the multimodality work of the New London Group and maintains that "anime films and videos can be extremely useful tools for teachers in implementing the vision of the multiliteracies theorists" (67)

One of the activities recommended in combination with anime is that of storyboarding. In filmmaking, storyboards are a sort of visual script that present in chronological order the still images of a film sequence. Storyboards look very much like comics, but the images usually are gestural or unfinished in appearance.

Hocks also uses/talks about storyboarding, but specifically as a scaffold for web design: "To teach
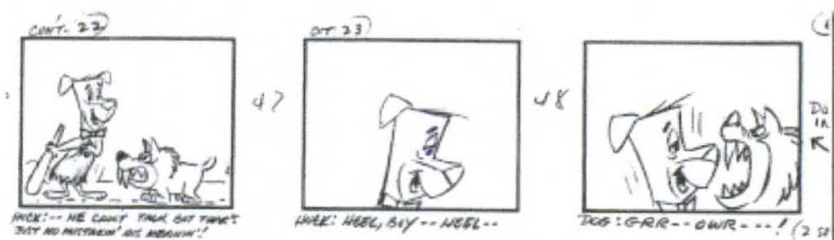

students, the storyboarding

process, I give them sheets of

paper and ask them to draw every
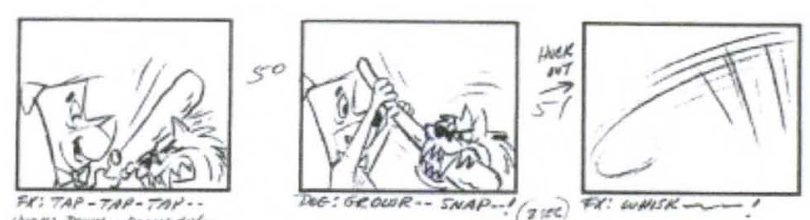

media element, each navigational

link, and all the text that appears
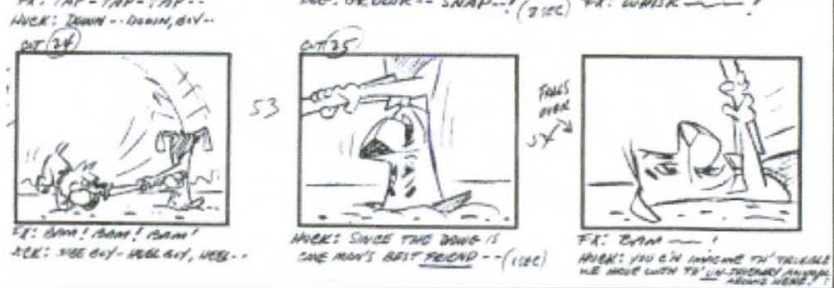

on screen. They also note the

colors and any other graphics that will be used on each screen. This process makes them pay careful attention to visual arguments, to spatial placement on the screen, and to the consistency of the interface" ("Understanding" 651). Storyboarding (like Sirc's "Basic Box") could then be a self-contained activity or a step in another process. The storyboard 
provides the visual grammar of a text, mapping the rhetorical principles that are displayed and disciplining the composer to present clear connections and expression in order to shape the viewer/reader experience that is a key factor in the ultrakinetic.

A useful resource for considering the possibilities of a storyboard-based assignment is Scott McCloud's 24 Hour Comics. McCloud recounts the genesis of the idea as a practical one. Both he and fellow comics artist Steve Bissette were struggling with timebased productivity and were looking for a way to inspire themselves to draw faster. The two challenged one another to complete an entire 24 page comic in the span of 24 consecutive hours. A sort of higher-order storyboarding, the 24-hour comic forces the

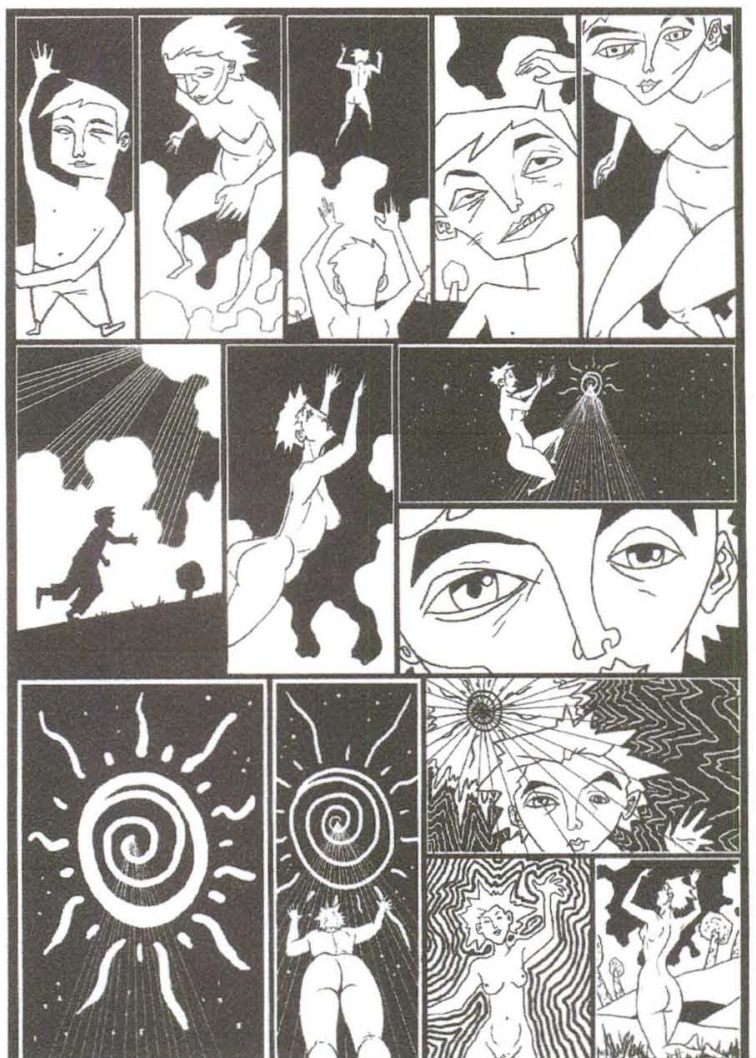

From Davison's 24 hour comic, "The Invisible Library"

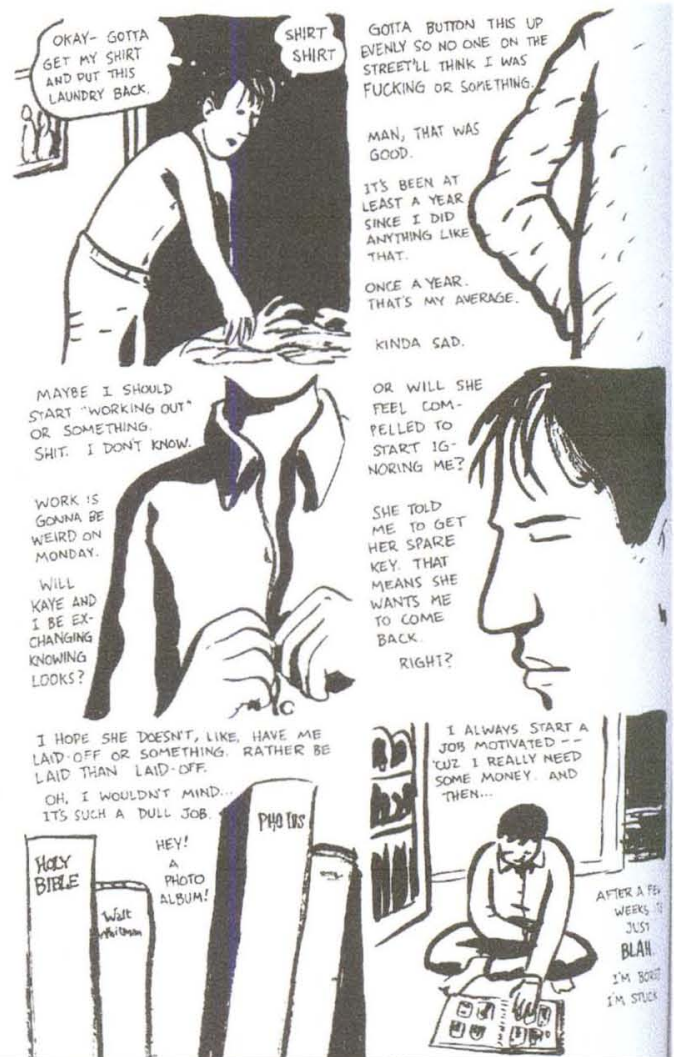

From Lasky's 24 hour comic, "Minutiae" 
composer to concentrate on the essence of both message and image; there is not enough time in the parameters of the endeavor of the 24 hour comic to perfect any component. The focus, then, remains on the experiential intent of the message.

What would a classroom version of a 24-hour comic include? One possibility would be to have students produce a storyboard in a single class session. Or the composition could be confined to another time period, perhaps the sum total of a week's worth of class meetings. The time constraint would encourage students to focus on content and construction in the same way the 24-hour comic forced McCloud to make composing decisions that were immediate and decisive. The time-constraint storyboard could be a method for jump-starting or interrupting non-productivity in a writer/composer who has become stuck (as we all do at some time).

Another variation on the storyboard is inspired by artist Marc Johns, who creates simple, usually humorous designs on Post It notes. In a comment regarding the Post It compositions (also called Sticky Note Drawings), Johns muses that “it's not so much about the craft of drawing, but more about the idea, boiled down to just the essentials. Again, students have an example of staying true to expressing whatever is vital in their argument as the critical portion of their composing process.
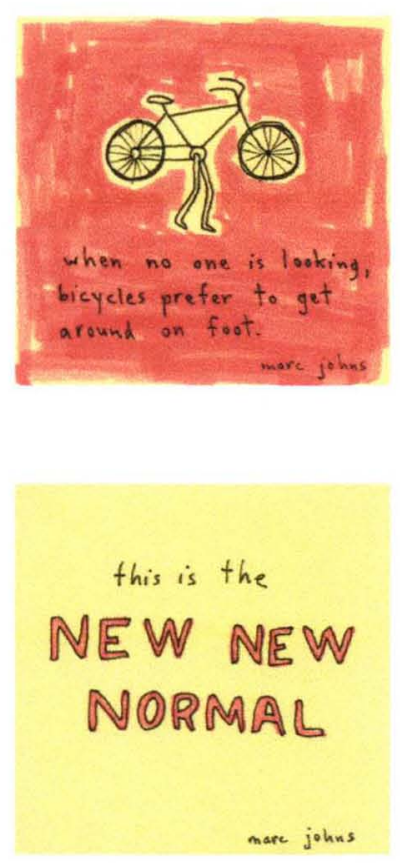
How would this work in a story board? Instead of using some other medium (large paper, dry erase or chalk board, a computer design program), students can be directed to confine each frame in their storyboard to a standard Post It note. Johns describes the limitation as deceptively challenging: "nothing like trying to cram an idea into nine square inches." Johns first collection of art, Serious Drawings (published in May 2009), would make an interesting (and fun!) addition to

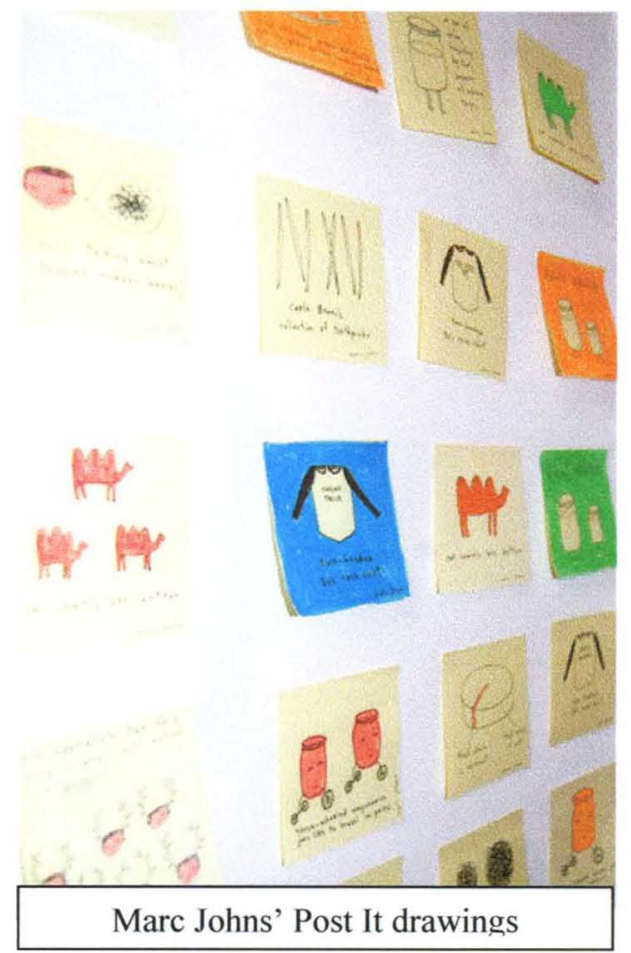
our visual rhetoric bookshelf that also includes Bang's Picture This and Berger's Ways of Seeing.

\section{Follow the Visual Road: Mapping}

Since multimodal compositions are nearly omnipresent, it is important to look to a variety of disciplines for appropriate pedagogical inspiration. In the field of business, there is growing buzz regarding the practice of "graphic recording" or professional visual notetaking. Graphic recording is a method of providing mediation for multipleparticipant meetings in which a facilitator writes and draws the cumulative ideas discussed. Graphic recording is a version of mind-mapping, a technique in which ideas and their interaction are represented visually on the page (or screen, or board). For example, artist Austin Kleon shares online his mind-maps of books he has read. Berger's Ways of Seeing is one: 


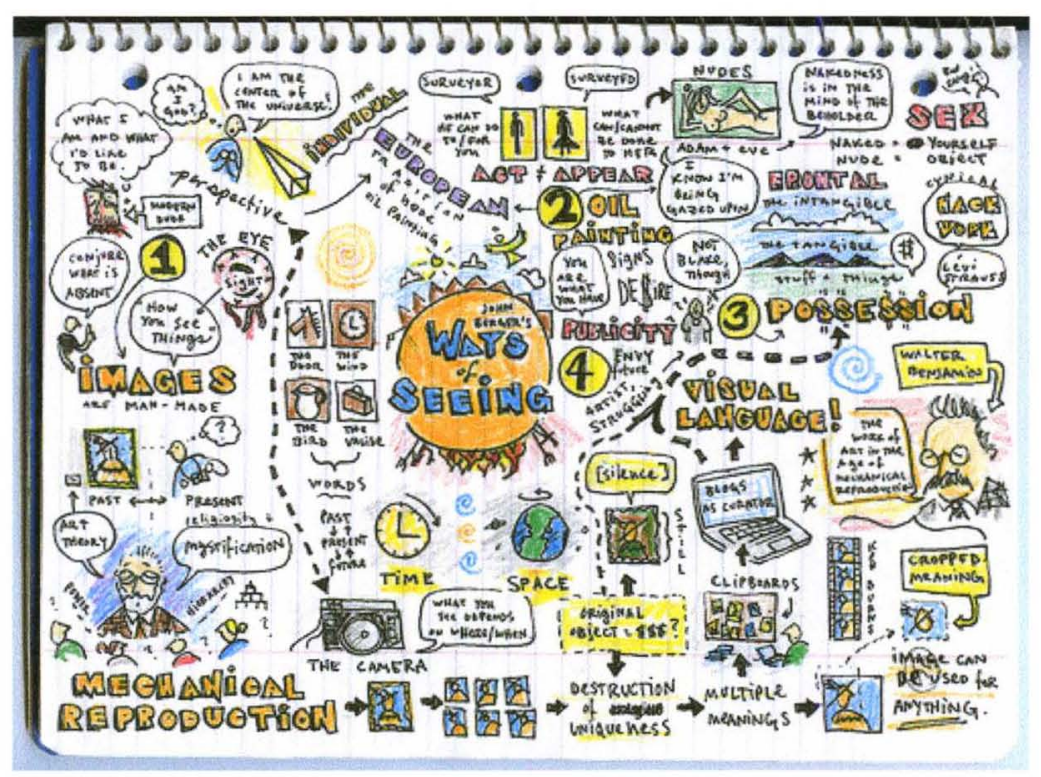

Note that Kleon scans his notes exactly as is from an ordinary spiral-bound lined notebook. Since many students display some sort of scribbling or doodling activity in class, focusing that attention into a directed process seems a viable means of teaching visual composition. The artistic ability required is come-as-you-are, find your own methods. In Writing New Media, Wysocki describes an assignment in which students are asked to compose using crayons, then analyze how the crayon-writing affected their process physically and creatively. Visual mapping or graphic recording could work well for the building of web pages as expressed by Sirc in "Box Logic."

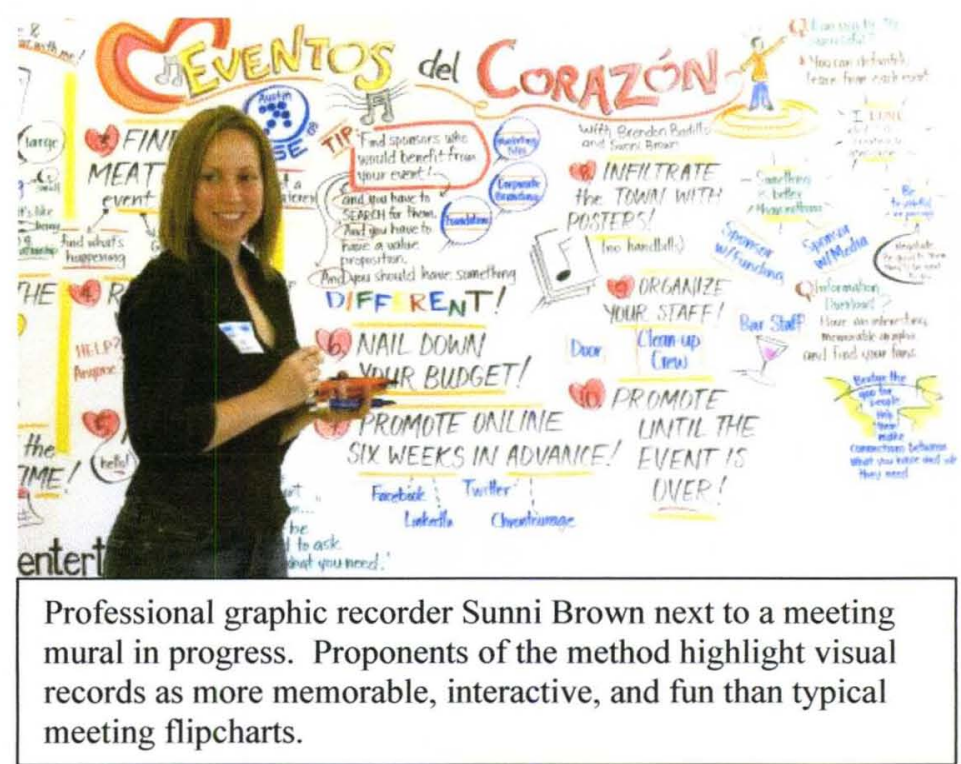


In another assignment included in Writing New Media, Selfe suggests that for the purposes of group interaction and critique, composers should label each of their images numerically in an activity constructed entirely with visuals (89). Some writer/composers may feel that the addition of the numbered labels adversely affects the aesthetic of the overall design. Thus in the spirit of providing both an experience and a suitable means of readily referencing specific aspects during discussion, the writer/composer could produce his or her designed text and an additional map or graphic record that would clearly delineate a vocabulary for reference to all of the design features. The production of such a map may also provide further transparency regarding the overall visual rhetoric of the piece, by providing additional anecdotal information or thoughts from the composer.

\section{Developing Visual Rhetoric Pedagogy}

A fundamental goal of education is to teach effective communication. It is the message that lies at the heart of communication, be it verbal, written, or pictorial, and the challenge to any communicator is to create accurate messages and interpret the messages of others with skill.

(Frey and Fisher Teaching Visual Literacy 1)

Some of the best recipes are those that are the conglomeration of other recipes: that one said a pinch, this one a dash: I use a teaspoon. In the effort to continue and expand the struggle that others have started, this chapter surveys my own idiosyncratic selection of visual rhetoric pedagogy. Part of my attraction to the assignments in Writing New Media clearly is that they can be defined as Anti-Essays, and therefore at least partially validate the impetus to formulate that assignment. Though Frey and Fisher's collection of 
practical classroom applications is intended for an audience of K-12 educators, ChandlerAlcott's determination that "sensitively planned classroom instruction around anime texts can nudge students toward more sophisticated meaning-making" (68) is relevant to my classroom as well. Similarly, her proclamation that "students who explore anime in a more formal way aren't the only ones who are likely to look at their worlds and themselves in a new way" (87) not only intimates how anime-inclusive pedagogy can change/teach, it echoes Selfe's desire for instructors to take the risk to be educated by their students, especially in effort to address and embrace multiple modalities.

In Pedagogy of the Oppressed, Freire mandates that "only through communication can human life hold meaning" (77). Such a declaration characterizes the work of composition as vital to the being of our students because we endeavor to help them communicate effectively. Effective communication has evolved even within our lifetimes to include the dizzying array of multiple modes and literacies frequently referenced in my text as well as the work of so many of my contemporaries. If, as teacher/learners of composition, we do not strive to incorporate the modes that are demanded by the world at large we lose a way to change the lives of our students.

$\gamma_{\text {It }}$ the reasons I advocate for ultrakinetic anime is the breadth and scope encompassed by such texts in terms of artistry, subject matter, and intertextuality. By using assignments like the Anti-Essay, students have the opportunity to explore, analyze, react and reflect in a multimodal manner that directly responds to the film in an evocative, interactive and 
experiential way. Thus the writer/composer does not merely relay information and/or formulate an argument; he or she creates an experience (Sirc might term a "happening.") The spice that incites the greatest impact may be practicing visual rhetoric principles in Molly Bang's “Arranging Shapes on a Rectangle." Or it could be that Sirc's box composition is the hot sauce students need. The chance to be the discerning collector or the purveyor of the fun factor present in modes like music and the ultrakinetic are roles that likely provide satisfaction. For other students, the close connection between a method like graphic recording and their future professional communication is the key ingredient.

So the visual rhetoric pedagogy recipe should be: inclusive, receptive, accessible and challenging, with methods and techniques that address a range of performance capability in a variety of modes and combinations of modes, always with the goal of developing the communication that connects and humanizes. 


\section{Chapter 5}

\section{Implications for Research and Practice in Visual Rhetoric}

Exploratory question: What if we use anime texts in Composition?

Research question: What is the impact and efficacy of using anime texts with composition students?

Schon's expression of the intuitive, almost instinctive impulse we as thinking beings share for reflection on our actions prompts instructors, students, readers, writers, and composers alike to address their work as the effort of skilled practitioners. Reflective practice is not relegated to specific fields; theory and practice intertwine in all areas, all modes, all literacies. Furthermore, Schon describes the informal inquiry of reflective practitioners as a viable form of experiment, termed exploratory experiment:

This is much of what an infant does when he explores the world around him, what an artist does when he juxtaposes colors to see what effect they make, and what a newcomer does when he wanders around a strange neighborhood. It is also what a scientist often does when he first encounters and probes a strange substance to see how it will respond.

Throughout this text, simple design elements help add a sense of visual cohesion to an argument that ranges across several chapters but seeks to maintain focus. The color design includes cool tone blues and greens, frequently presented with some transparency rather than full saturation in order to render what are naturally soothing shades softer. Plain geometric shapes are also repeated: circles, rectangles (sometimes square), and triangles. This presents a visual logic that the content is composed at least in part of building blocks. The green thread that is scribbled across and beside text draws attention to specific sections and/or connects words, phrases and/or names together; it also references the poem "The Force that Through the Green Fuse Drives the Flower" by Dylan Thomas.

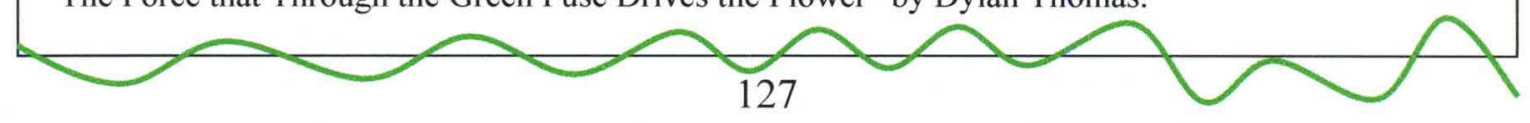


In many ways, this dissertation is an exploratory experiment in the area of visual rhetoric, with a specific focus on the ultrakinetic and anime. McCloud conveys a similar affinity for the idea of experiment when he admires the combined visual and literate (image and word) work of artists and writers whose chosen form is comics. Comics practitioners are participating in the process of exploratory experiment as described by Schon. Voices from disparate contexts combine as one on this subject when Freire joins Schon and McCloud and echoes the importance of what is, in essence, reflective practice in the caution that "Those who authentically commit themselves to the people must re-examine themselves constantly" (60). I contend that the phrase "to the people" in Freire's statement may seem specific to the social and political unrest from which he wrote, but the people may be our students, our peers, fellow artists, exploratory scientists and composers.

In Chapter 2, I anchor the study presented by this dissertation not only in the principles of reflective practice, but also significantly in Carini's desire to see education (and our culture as a whole) centered on an idea of humanness. Such a pedagogy (social/cultural structure) allows for decision making that is open, inclusive and considerate. Power, control or authority for one's self (read also
Carini's "Meditation: On Description" also serves as an apt description

(repetition/reference intended) of a manner in which the reflective practitioner may observe, record, and respond to her actions:

"Describing I pause, and pausing, attend. Describing requires that I stand back and consider. Describing requires that I not rush to judgment or conclude before I have looked. Describing makes room for something to be fully present. Describing is slow, particular work. I have to set aside familiar categories for classifying or generalizing. I have to stay with the subject of my attention. I have to give it time to speak, to show itself" (163).

In some ways, reflection necessitates a sort of listening first. 
agency) should not be seated outside or

even wholly inside the individual. Within

a pedagogy of humanness, each person has

the right and responsibility to be an active

agent in the most essential tool of

education: communication. Freire frames

his own delineation of what he calls

humanization in "a humanist and

libertarian pedagogy" (54). Individuals

are set free by joining together in

reflective practice:

\section{A revolutionary leadership must}

accordingly practice co-intentional

education. Teachers and students

(leadership and people), co-intent

on reality, are both Subjects, not

only in the task of unveiling that

reality, and thereby coming to

know it critically, but in the

task of re-creating that

knowledge. As they attain this

knowledge of reality through

common reflection and action, they
Looking [Fast] Forward

Some discussion of contemporary communication is wholly focused on the quickening of our interactions. For example, in her discussion of anime, Napier notes that:

"With its rapid shifts of narrative pace and its constantly transforming imagery, the animated medium is superbly positioned to illustrate the atmosphere of change permeating not only Japanese society but also all industrialized or industrializing societies. Moving at rapid-sometimes breakneck - pace and predicated upon the instability of form, animation is both a symptom and a metaphor for a society obsessed with change and spectacle" (12).

Garoian and Gaudelius emphasize the need for critical understanding of "the spectacle pedagogy of visual culture" (1). They also encourage student artists to participate in the creation of "counterspectacle that challenges the virulent power of mass culture" (6).

In contrast to Napier's view, Gleick provides a qualifier to his own overall argument when he reminds us that change is evident in all things, even those we think we know well:

"The book-even a book called Faster-has become a notoriously slow device: slow in the writing, slow in production, slow to read and absorb. That was not always true. The printed word began as advanced technology for rapid transmission of data into the brain" (283).

Schon, like Gleick, understands and describes the different "speeds" of interaction. He notes: "a practitioner's reflection-in-action may not be very rapid" (62) though an example of jazz musicians highlights how they "manifest a 'feel for' their material and they make on-the-spot adjustments to the sounds they hear. Listening to one another and to themselves, they felt where the music is going and adjust their playing accordingly" (55).

Manipulation of the speed of motion describes and is referenced by a world of rapid changes in communication, technology and culture.

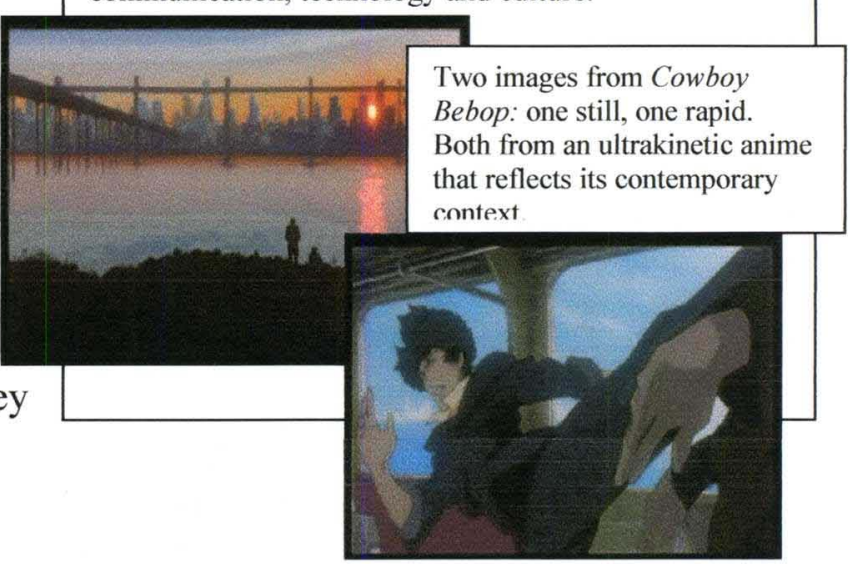


discover themselves as its permanent re-creators. (Freire 69)

This dissertation helps to create a vocabulary and method with which to discuss ourselves and our students in the contemporary classroom. Sirc struggles to present his ideas beyond the limitations of print while at the same time laboring to preserve an individualistic human quality in his students, who as imperfect beings may respond best to a variety of opportunities for expression, including imperfect ones (i.e. Duchamp's imperfect "readymade" art and student Greg White's error-ridden but evocative prose). Sirc uses 1960s art happenings as a model for how we should be providing, or at the very least, allowing experiences in the composition classroom. Marrying Sirc and Schon together I propose an exploratory experiment: the viewing/reading of ultrakinetic texts in the composition classroom providing experiences and instruction for students who prepare for a rapidly changing technological and literate world.

Ultrakinetic anime texts are just one form for study, a way to focus, but one that offers unique points of discussion and reaction or experience. Asian studies professor Thomas

Lamarre helps situate anime's academic inclusion as part of the results of the new media explosion, explaining that "the
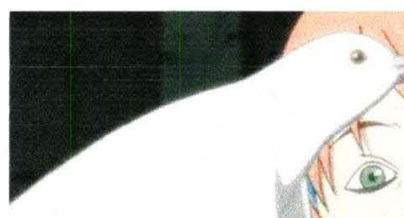
it

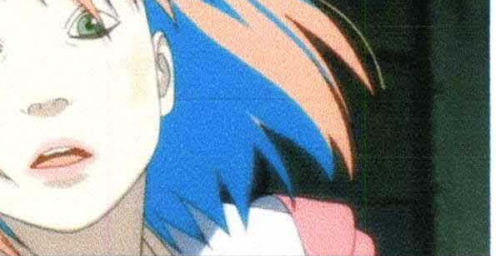

Still from The Animatrix. The nine short films, all ultrakinetic anime based on the live action feature film The Matrix (also ultrakinetic), total 89 minutes of viewing time. This makes the text very usable in the time-restricted confines of a class period. Classes could view (read) one of the films together and have time for discussion and/or written response.

emergence of new media not only forces us to re-think what cinema is, but also promises new ways to think about something like anime" (332). "New ways" to think are 
necessary because of the changing (or changed) nature of texts we read/view and write/compose.

Despite the positive impact I see in the use of ultrakinetic anime texts, there are issues to be addressed regarding their inclusion in the composition classroom. The common concerns or possible counterarguments expressed are the result of the assumption that the films are dominated by images and themes of sex and violence and therefore not fit for education. First, not all anime is "adult" in theme; there is a broad

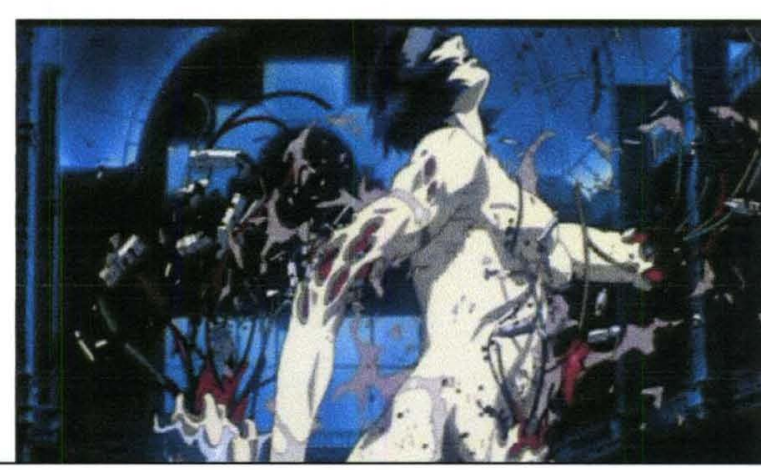

From Ghost in the Shell: nudity and violence... it must be anime.

Certainly there are anime that would not be suitable for certain audiences, but it's not all sexy violence. Also, it is important to note that extreme images of sexuality and/or violence frequently have a subject that is other than human. Here, Kusanagi is a cyborg. In the film Metropolis, one of the more violent acts has a robot for a victim.

range of subject matter and target audience throughout. To limit Japanese animation to a pornographic designation is as accurate as terming all American animation as meant for toddlers. Second, some of the more adult themes may be perfectly appropriate in the college environment. I am not necessarily advocating for hardcore pornographic anime to be daily classroom fuel, but I do recognize the critical analysis made possible in the discussion of Berger's visual essay in which so-called pornography is juxtaposed with images of art.

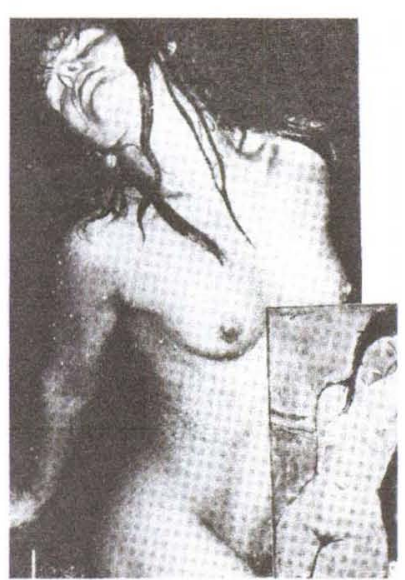


Critic Susan Napier directly addresses value in pornographic anime, that it "brings up obvious questions of gender construction and interaction, but also less obvious ones as well, such as the relation of gender, power, and control to technology, tradition, and transition" (64). I believe there are quite a few individuals who would be surprised by Napier's subsequent assertion that "the pornographic in anime turns out to be a deeply conservative genre at heart, offering its male viewers visions of fantasy identities integrally linked to a lost traditional culture" (83). In Anime Explosion, Drazen concurs, offering that "sex is a part of life and, in Japan, a part of pop culture. But manga and anime know how to add variety and keep things fresh: by lacing sex with humor, with horror, and with old-fashioned sentimentality" (59). Conscientious, unflinching and intentional examination like that of Napier and Drazen helps couch any concern that some may have regarding the inclusion of anime as the encroachment of pornography into the classroom. Not only does anime range throughout a variety of subjects and themes, an enormous selection of which are not explicit, the films and series that are PG13 and R-rated may be well worth academic consideration.

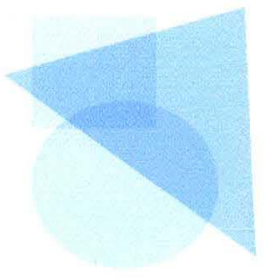

Another possible caution for the inclusion of anime may be that other sources could be just as (or more) effective in generating student response. In her look at anime in secondary education, Chandler-Olcott identifies useful methods, but she is careful to provide her own counter-argument: "There are plenty of other forms of popular culturegraffiti art, stepdancing, computer gaming, just to name a few-that have been embraced by young people while remaining marginalized in formal academic settings. Any number 
of these pursuits might be more appropriate choices for opening up the curriculum and expanding definitions of literacy in a given classroom than anime" (86). Of course there are always other choices (see Sirc or Hess for detailed accounts of the productive inclusion of hip hop and rap music, for example), but like Chandler-Olcott, I make a case for why anime is effective.

One of the parameters of ultrakinetic anime is that the visuals suggest layers of meaning within a presentation of overall information that is densely packed. Such a text serves as an effective model for composing a similarly complex visual and literate message. The gun control collaborative Anti-Essay presented in Chapter 4 combines the authors' personal narratives regarding guns and gun regulation with the not-so-secondary purpose of questioning the inherent authority of the book format. Keri Smith's Wreck This Journal is a mainstream publishing expression of the argument the gun control group developed organically. Smith provides directives like "burn this page," "throw something dipped in paint," and "draw outside the lines." Wreck This Journal is a sort of
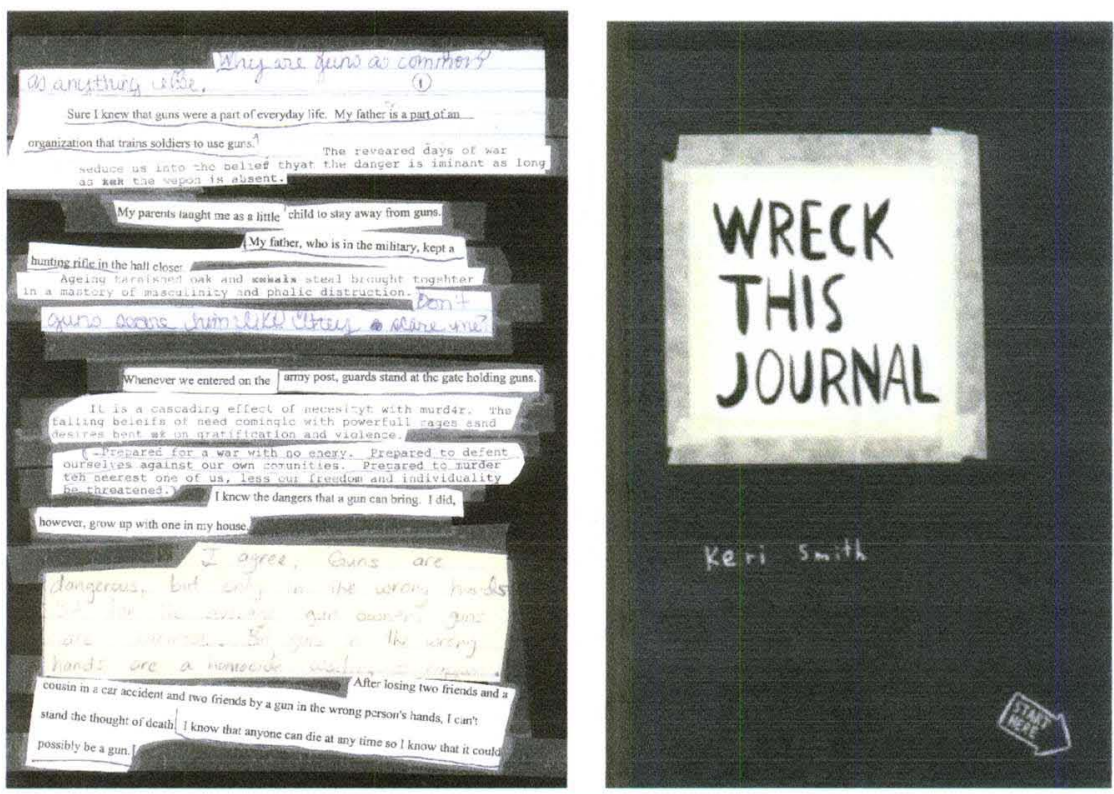

Like a proud "mom," I submit that the AntiEssay students wrecked their artifact with messy tape before Smith published hers.

(;) 
Anti-Essay paint-by-number that could provide some jump-start suggestions for students

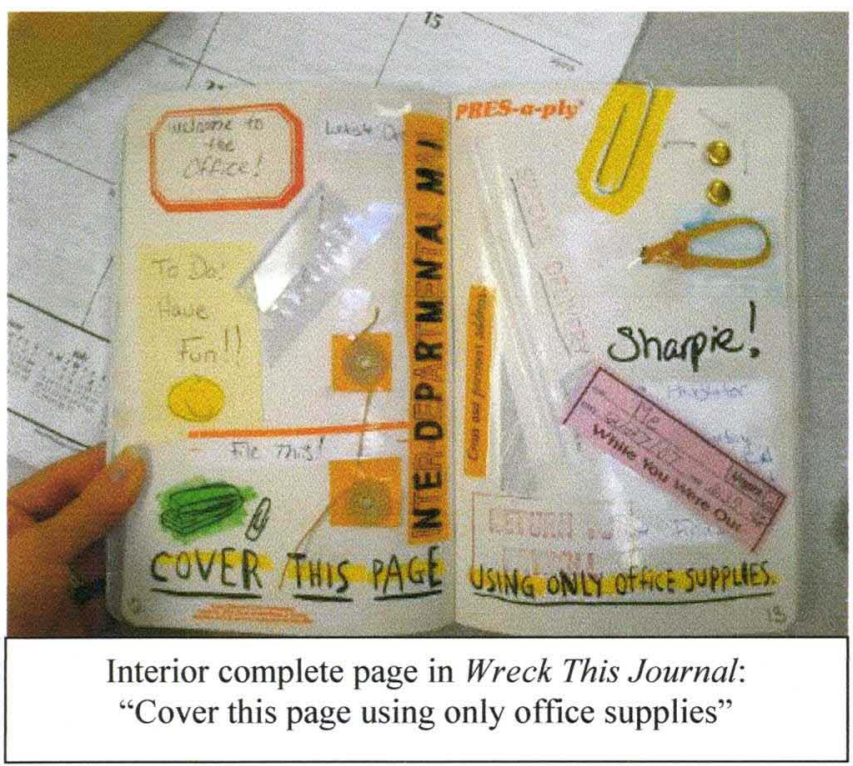

who struggle without clearly defined plans.

Another possible model for use in the classroom is found in The 1000 Journal Project. The 1000 Journal Project is an advanced shared-journal version of the Anti-Essay with an added ultrakinetic bonus: the journal physically travels to different countries. In the year 2000, a San Francisco based artist working under the name "Someguy" conceived and initiated a multimodal experiment in which 1,000 blank journals were sent to individuals or randomly left in a variety of locations with the instructions: "One thousand journals are traveling from hand to hand throughout the world. Those who find them will add their stories and drawings, and then pass the journal along in an ongoing collaborative art form. This is an experiment, and you are a part of it. You have 24 hours. Read it. Add to it. Pass it on." The exploratory experiment 1000 Journals Project produces

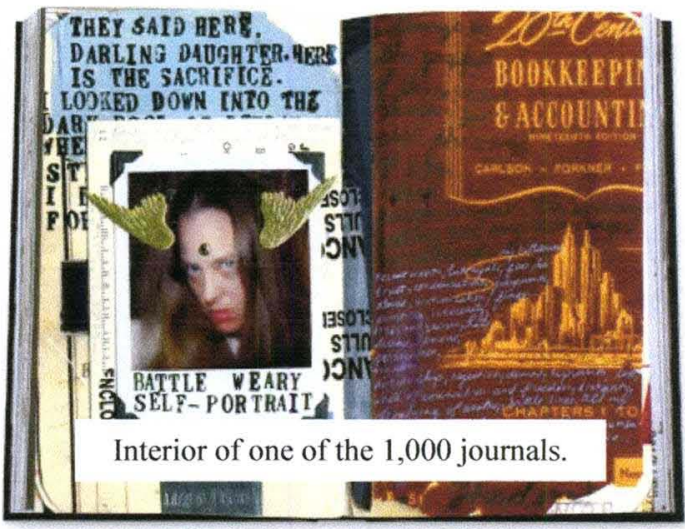
ultrakinetic visual and literate texts (some also reference, due to individual writer/composer interest, anime-related content, art or covers. The journals project is 
easily replicable in a classroom environment, and would invite participation from all

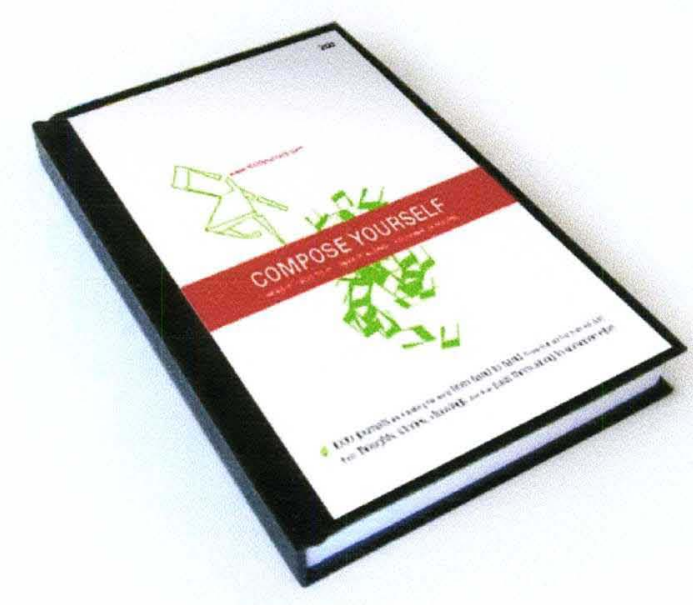

Journal \#260: "Compose Yourself" cover designed by artist Anders Hornstrup students despite predictably varying

degrees of comfort and facility with

artistic expression. The opportunity for

interactive, collaborative text production is

intrinsic to this format and a dynamic way

for students to explore some of the

complexities and nuances of

communication, particularly in a world in

which "sharing" has become so

commonplace. This making the private public is seen clearly in the constantly accessible "status updates" and other information that individuals post on Facebook or Twitter. By contributing to and analyzing the results of a collaborative art journal, students are practicing the ability to purposefully generate and subsequently investigate texts that are a part of our current culture.

One outcome of this dissertation is the observation of how the language (label?) we use to describe who communicates and how they communicate has changed. Selfe uses viewer/reader where reader once applied and writer/composer where writer used to suffice. In his 2006 Making Comics (the second successor to Understanding Comics), Scott McCloud prefers maker to either artist or writer to express the meaning-making the designer accomplishes. He also uses make and create interchangeably. In addition to the phrase active agent, Carini, like McCloud uses the label of maker as one that helps 
encompass word, image, and thoughtful analysis in text production by human beings of all ages (and therefore of great importance): "I propose, by this bringing together in one frame of the works of artists and children, a continuum of makers I believe worthy of our educational, social, and political attention" (22): "In Writing New Media, Wysocki chooses to use composer consistently to describe the individual who creates a text:

We should call 'new media texts' those that have been made by composers who are aware of the range of materialities of texts and who then highlight the materiality: such composers design texts that help readers/consumers/viewers stay alert to how any text-

In his "Box Logic," Sirc suggests that a collector is a composer as well.

like its composers and readers_-doesn't function independently of how it is made and in what contexts. Such composers design texts that make as overtly visible as possible the values they embody. (15)

In contemporary college culture it is not: students write. It is: composers design. The term writing does not encompass entirely the kinds of composing performed both in and out of the classroom. Changing culture in school, business, and social communication requires a new "composing" education for college students to be literate and effectively create the documents of the present and the future.

In order to address the question of why visual rhetoric, why now, we must look at changing texts and communication methods. Part of the business of composition now involves determining how our "the written text now has to look good" (65)

Kress Literacy in the New Media Age classrooms can react, how we will reflect and include the real world. Sirc looks very 
specifically at business communication that is typical in the office space, but not usually included in study:

Future businesspeople, for example, might profit by becoming more attentive to email gossip, how to read it, how that writing fits in our culture, whence it arises, and how it represents reality (indeed, the very reality it chooses to represent). It might give students a better sense of control over their futures, show them a side of their future profession that the textbooks don't, show them the spectacular (say, Dynasty, Survivor) just might be a more instructive text for the way business writing actually works that the professional. (Happening 220).

In their recent WPA: Writing Program Administration article "Composing in a Digital World: The Transition of a Writing Program and Its Faculty" Takayoshi and Huot recall computer company executives' response to a successful proposal, which included a student-produced movie, for $\$ 450,000$ in computers and classroom electronics. While the reps listened solemnly to the presentation of curricular goals and theoretical arguments, minutes into the presentation of the student video project, they had eagerly leaned forward in their chairs, asking questions, offering their response, and engaging with the theoretical issues in substantial understanding. One representative commented, 'I have a staff of 30 people under me-and I wish all of them knew how to make such a composition." (192)

The applicable point here to visual rhetoric pedagogy is that we need to consider new texts and that consideration should defer to some practicalities of communication for the 
preparation of the contemporary student. Napier helps describe why anime may be just such a practical resource: "It may be animation in general—and perhaps anime in particular - is the ideal artistic vehicle for expressing the hopes and nightmares of our uneasy contemporary world" (11). By presenting students visual and literate texts to read, it expands not only the skill of processing and interpreting texts, it also flexes the ability to perform exploratory experiments in the texts they choose to compose. Wysocki remarks:

Ask students to imagine that they'd spent as much time learning to drawing or to manipulate photographic images [as learning to read and write]. What do they think their attitudes toward drawing or photographs would be? How might texts be different? (Writing New Media 28)

The question isn't if texts would be different; the question is how texts would be different. The suggestion is that texts should be different.

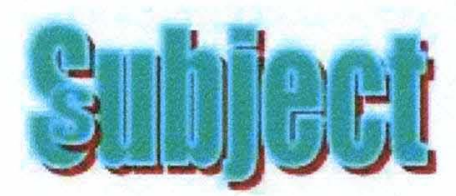

Because a visual rhetoric pedagogy that includes ultrakinetic texts is experiential, it creates a classroom atmosphere that welcomes, if not encourages, interactive, subjective thoughts and responses. With an ultrakinetic visual rhetoric pedagogy we incite students to be Carini's active agents and Freire's human re-creators. Reflective practice is something our students should be doing. The inclusion of ultrakinetic anime in a visual rhetoric pedagogy is an effective, evocative, idea generative and expressive method of creating an experience for students in which they may practice reading, explore modes of composing, and become critical thinkers and communicators suited for the $21^{\text {st }}$ century. 
To be continued by

composers... 


\section{REFERENCES}

Akira. Dir. Katsuhiro Otomo. Pioneer, 1987.

Anderson, Charles M. "In Search of a Visual Rhetoric for Instructional Television." $A V$ Communication Review 20.1 (Spring 1972): 43-63.

The Animatrix. Warner Bros., 2003.

Aristotle. The Art of Rhetoric. New York: Penguin, 1991.

Arnheim, Rudolf. Visual Thinking. Berkeley: U of California P, 1969.

Avinger, Charles. "Visual Literacy, Critical Thinking and Composition: An Integrated Approach." Pennsylvania English 19.1 (1994): 1-12.

Ball, Cheryl E. and Kristin L. Arola. IX: Visual Exercises. Boston: Bedford St. Martin's, 2004.

Bang, Molly. Picture This: Perception and Composition. Boston: Little Brown \& Co., 1991.

Barthes, Roland. Image Music Text. Trans. Stephen Heath. New York: Hill and Wang, 1977.

Barton, Ben and Marthalees Barton. "Toward a Rhetoric of Visuals for the Computer Era." Technical Writing Teacher 12 (Fall 1985): 126-45.

Baudrillard, Jean. The Ecstasy of Communication. New York: Semiotext(e), 1988.

Belton, John, ed. Movies and Mass Culture. Rutgers UP, 1996.

Berger, John. Ways of Seeing. London: Penguin, 1972.

--- and Jean Mohr. Another Way of Telling. New York: Vintage, 1995. 
Bernhardt, Stephen. "Seeing the Text." CCC 37 (1986): 66-78.

---. "The Shape of Text to Come: The Texture of Print on Screens." CCC 44 (1993): $151-75$.

Bishop, Ellen. Cinema-(To)-Graphy: Film and Writing in Contemporary Composition Courses. Portsmouth: Boynton/Cook, 1999.

Blackmon, Samantha. “ 'But I'm Just White' or How 'Other' Pedagogies Can Benefit All Students." Teaching Writing with Computers. Eds. Pamela Takayoshi and Brian Huot. Boston, MA: Houghton-Mifflin, 2003: 92-102.

Blakesley, David and Collin Brooke, eds. Enculturation 3.2: Visual Rhetoric. http://www.uta.edu/huma/enculturation/3_2/

Block, Bruce. The Visual Story: Seeing the Structure of Film, TV and New Media. Boston: Focal Press, 2001.

Blood: The Last Vampire. Dir. Hiroyuki Kitakubo. Manga Entertainment, 2000.

Bolter, Jay David and Richard Grusin. Remediation: Understanding New Media. Cambridge: MIT Press, 1999.

Braudy, Leo and Marshall Cohen, eds. Film Theory and Criticism. $5^{\text {th }}$ ed. New York: Oxford UP, 1999.

Brooker, Peter and Will, eds. Postmodern Afterimages. Boston: Bedford, 1997. Brown, Steven T. Cinema Anime. New York: Palgrave Macmillan, 2006.

Buckingham, David. Media Education: Literacy, Learning and Contemporary Culture. Malden, MA: Polity Press, 2003. 
Bukatman, Scott. Terminal Identity: The Virtual Subject in Postmodern Science Fiction. Durham: Duke UP, 1993.

Burgess, Anthony. A Clockwork Orange. New York: Norton \& Co., 1962.

Campbell, Kermit E. 'There Goes the Neighborhood: Hip Hop Creepin' on a Come Up at the U." CCC 58.3 (Feb 2007): 325-344.

Caputo, Tony C. Visual Storytelling: The Art and Technique. New York: Watson Guptill, 2003.

Carini, Patricia F. "Dear Sister Bess: An Essay on Standards, Judgment, and Writing." Assessing Writing 1 (1): 29-67.

---. Starting Strong: A Different Look at Children, School and Standards. New York: Teachers College Press, 2001.

CCCC San Antonio. Making Composition Matter: Students, Citizens, Institutions, Advocacy. Urbana, IL: NCTE, 2004

Childers, Pamela, Eric Hobson and Joan Mullin, eds. ARTiculating: Teaching Writing in a Visual World. Portsmouth, NH: Boynton/Cook, 1998.

Clements, Jonathan and Helen McCarthy. The Anime Encyclopedia. Berkeley: Stone Bridge Press, 2001.

Cope, Bill and Mary Kalantzis. Multiliteracies. New York: Routledge, 2000.

Cowboy Bebop: The Movie. Dir. Shinichiro Watanabe. Tristar, 2003.

Cowboy Bebop: The Series. Bandai Entertainment, 1999.

Crary, Jonathan. Suspensions of Perception: Attention, Spectacle, and Modern Culture.

Cambridge: MIT Press, 2000.

Curtis, David. Experimental Cinema. New York: Dell, 1971. 
Dancyger, K. The Technique of Film and Video Editing. Boston: Focal Press, 1993. Darley, Andrew. Visual Digital Culture: Surface Play and Spectacle in New Media Genres. New York: Routledge, 2000.

Danino, Nina and Michael Maziere, eds. The Undercut Reader: Critical Writings on Artist's Film and Video. London: Wallflower, 2003.

Desser, David. "Blade Runner: Science Fiction and Transcendence." Literature/Film Quarterly 13.3 (1985): 173-78.

Dick, Bernard. Anatomy of Film. Boston: Bedford, 2002.

Donnie Darko. Dir. Richard Kelly. 20 $0^{\text {th }}$ Century Fox, 2001.

Douglas, J. Yellowlees. The End of Books - Or Books without End?: Reading Interactive Narratives. Ann Arbor: U of Michigan P, 2001.

Dreams. Dir. Akira Kurosawa. Warner Bros., 1990.

Druckery, Timothy, ed. Ars Electronica: Facing the Future. Cambridge: MIT Press, 2001.

Eisner, Will. Comics and Sequential Art. Tamarac, FL: Poorhouse Press, 1985.

---. Will Eisner's Shop Talk. Wilwaukie, OR: Dark Horse Comics, 2001.

Elkins, James. On Pictures and the Words that Fail Them. Cambridge: Cambridge UP, 1998.

Faigley, Lester, Diana George, Anna Palchik and Cynthia Selfe. Picturing Texts. New York: Norton, 2004.

Farris, Christine and Chris Anson, eds. Under Construction: Working at the Intersections of Composition Theory, Research and Practice. Logan: Utah State UP, 1998. 
Field, Simon. "Out of the Box." Sight and Sound 12.11 (2002 Nov): 5.

Fight Club. Dir. David Fincher. $20^{\text {th }}$ Century Fox, 1999.

Freire, Paulo. Pedagogy of the Oppressed. New York: Continuum Intl. Pub. Group, 2000.

Frey, Nancy and Douglas Fisher, eds. Teaching Visual Literacy. Thousand Oaks, CA: Corwin, 2008.

---. "Using Graphic Novels, Anime and the Internet in an Urban High School." English Journal 93.3 (January 2004): 19-25.

Fuery, Patrick. New Developments in Film Theory. New York: Palgrave Macmillan, 2000

Garoian, Charles R. and Yvonne M. Gaudelius. Spectacle Pedagogy: Art, Politics, and Visual Culture. New York: State U of New York P, 2008.

Garrett-Petts, W.F. and Donald Lawrence, eds. Integrating Visual and Verbal Literacies. Winnipeg: Inkshed, 1996.

George, Diana. "From Analysis to Design: Visual Communication in the Teaching of Writing." CCC 54.1 (Sept 2002): 11-39.

Ghost in the Shell. Dir. Mamoru Oshii. Polygram, 1996.

Gledhill, Christine and Linda Williams. Reinventing Film Studies. London: Arnold, 2000.

Gleick, James. Faster: The Acceleration of Just About Everything. New York: Pantheon, 1999.

Goulart, Ron. Comic Book Culture: An Illustrated History. Portland, OR: Collectors P, 2000. 
Grave of the Fireflies. Dir. Isao Takahata. Central Park Media, 2002.

Handa, Carolyn. Visual Rhetoric in a Digital World: A Critical Sourcebook. Boston: Bedford St. Martin's, 2004.

Halberstam, Judith and Ira Livingston, eds. Posthuman Bodies. Bloomington: IUP, 1995.

Hanhardt, John. Worlds of Nam June Paik. New York: Guggenheim Museum, 2000.

Harste, Jerome, Virginia Woodward and Carolyn Burke. Language Stories and Literacy Lessons. Portsmouth, NH: Heinmann, 1984.

Hawisher, Gail E. and Cynthia L. Selfe, eds. Computers and Composition Special Issues: Digital Rhetoric, Digital Literacy, Computers and Composition. 18.1,2 2001.

---. Passions, Pedagogies and $21^{\text {st }}$ Century Technologies. Logan, UT: Utah State UP, 1999.

Hayles, Katherine. How We Became Posthuman. Chicago: U of Chicago P, 1999. Hill, John and Pamela Church Gibson. Film Studies: Critical Approaches. New York: Oxford UP, 2000.

Hocks, Mary. "Teaching and Learning Visual Rhetoric." Teaching Writing with Computers. Eds. Pamela Takayoshi and Brian Huot. Boston: Houghton Mifflin, 2003.

---. "Understanding Visual Rhetoric in Digital Writing Environments." CCC 54.4 (June 2003): 629-54.

Hocks, Mary and Michelle Kendrick, eds. Eloquent Images: Word and Image in the Age of New Media. Cambridge, MA: MIT Press, 2003.

Howe, Susan. The Nonconformist's Memorial. New York: New Directions, 1993. 
Inge, M. Thomas. "Comic Books." The Greenwood Guide to American Popular

Culture. Volume 1. Eds. M. Thomas Inge and Dennis Hall. Westport, Conn: Greenwood P, 2002. 275-305.

Johnson-Eilola, Johndan. Nostalgic Angels: Rearticulating Hypertext Writing.

Norwood, N.J.: Ablex Pub., 1997.

Joselit, David. Jenny Holzer. London: Phaidon Press, 1998.

Joyce, Michael. Of Two Minds: Hypertext Pedagogy and Poetics. Ann Arbor:

University of Michigan Press, 1995.

Kellner, Douglas. Media Culture. New York: Routledge, 1995.

Kill Bill. Dir. Quentin Tarantino. Miramax, 2003.

Kress, Gunter. Literacy in the New Media Age. New York: Routledge, 2003.

Kumpf, Eric. "Visual Metadiscourse: Designing the Considerate Text." Technical

Communication Quarterly 9.4 (Fall 2000): 401-24.

Lamarre, Thomas. "From Animation to Anime: Drawing Movements and Moving

Drawings." Japan Forum 14.2 (2002): 329-67.

Loveless. Dir. Yumi Nakayama. Anime Works, 2007.

Lucie-Smith, Edward. Art Today. London: Phaidon Press, 1999.

---. Art Tomorrow. New York: Vilo International, 2002.

Lunning, Frenchy. Emerging Worlds of Anime and Manga. Minneapolis: $\mathrm{U}$ of Minnesota P, 2006.

MacWilliams, Mark, ed. Japanese Visual Culture. New York: East Gate, 2008.

Manovich, Lev. The Language of New Media. Cambridge: MIT Press, 2001. 
Marc, David. Bonfire of the Humanities: Television, Subliteracy, and Long-Term Memory Loss. New York: Syracuse UP, 1995.

Markel, Mike. "What Students See: Word Processing and the Perception of Visual Design." Computers and Composition 15 (1998): 373-86.

Martinez, D. P., ed. The Worlds of Japanese Popular Culture. New York: Cambridge UP, 1998.

The Matrix. Dir. Andy and Larry Wachowski. Warner Bros., 1999.

McCloud, Scott, ed. 24 Hour Comics. Thousand Oaks, CA: About Comics, 2004.

---. Making Comics. New York: Harper, 2006.

---. Reinventing Comics. New York: Paradox Press, 2000.

---. Understanding Comics. New York: Harper, 1993.

McQuade, Donald and Christine, eds. Seeing \& Writing. Boston: Bedford/St. Martin's, 2000 .

McQuarrie, Edward F. and David Glen Mick. "Visual Rhetoric in Advertising: TextInterpretive, Experimental, and Reader-Response Analyses." The Journal of Consumer Research 26.1: 1999.

Messaris, Paul. Visual Literacy: Image, Mind, and Reality. Boulder: Westview P, 1994. Metropolis. Dir. Fritz Lang. Madacy, 1926.

Micciche, Laura. "More Than a Feeling: Disappointment and WPA Work." College English 64 (2002): 432-58.

Miller, Frank. Batman: The Dark Knight Returns. New York: DC Comics, 1997.

Mitchell, W.J.T. Iconology: Image, Text, Ideology. Chicago, U of Chicago P, 1986. 
---. Picture Theory: Essays on Verbal and Visual Representation. Chicago: U of Chicago P, 1994.

Morse, Margaret. Virtualities: Television, Media Art and Cyberculture. Bloomington: Indiana UP, 1998.

Moulin Rouge. Dir. Baz Luhrmann. $20^{\text {th }}$ Century Fox, 2001.

Muth, Marcia F. and Karla Saari Kitalong. Getting the Picture: A Brief Guide to Understanding and Creating Visual Texts. Boston: Bedford St. Martin's, 2004.

Napier, Susan J. Anime from Akira to Princess Mononoke: Experiencing Contemporary Japanese Animation. New York: Palgrave, 2000.

Neon Genesis Evangelion: Death \& Rebirth. Dir. Hideaki Anno. Manga Video, 1997.

Neon Genesis Evangelion: The Series. ADV Films, 2005.

Nguyen, Dominic. "Rebirth of the Cool." Newtype USA 4.6 (June 2005): 8-13.

Ninja Scroll. Dir. Yoshiaki Kawajiri and Quint Lancaster. Manga Video, 1995.

Osamu Tezuka's Metropolis. Dir. Rintaro. Tristar, 2001.

Packer, Randall and Ken Jordan, eds. Multimedia: From Wagner to Virtual Reality. New York: Norton, 2001.

Patten, Fred. Watching Anime, Reading Manga: 25 Years of Essays and Reviews. Berkeley, CA: Stone Bridge Press, 2004.

Peacock, Richard Beck. The Art of Movie Making. Upper Saddle River, NJ: PrenticeHall, 2001.

Penny, Simon, ed. Critical Issues in Electronic Media. Albany: SUNY Press, 1995. 
Phelps, Louise W. "Images of Student Writing: The Deep Structure of Teacher Response." Writing and Response: Theory, Practice, and Research. Ed. Chris Anson. Urbana, IL: NCTE, 1989: 37-67.

Pilling, Jayne, ed. A Reader in Animation Studies. Bloomington: IUP, 1997.

Poitras, Gilles. The Anime Companion. Berkeley: Stone Bridge Press, 1999.

---. Anime Essentials. Berkeley: Stone Bridge Press, 2001.

Porter, James and Patricia A. Sullivan. "Repetition and the Rhetoric of Visual Design." Advances in Discourse Processes 48: 1994.

Poster, Mark. The Second Media Age. Cambridge: Polity Press, 1995.

Princess Mononoke. Dir. Hayao Miyazaki. Buena Vista, 1997.

Pulp Fiction. Dir. Quentin Tarantino. Miramax, 1994.

Ramey, John Wilson. The Confluence of Visual and Verbal Rhetoric: Toward a Pedagogical Theory of the Imagetext. University of Louisville, August 2000.

Ranker, Jason. "Designing Meaning with Multiple Media Sources: A Case Study of an Eight-Year-Old Student's Writing Processes." RTE 41.4 (May 2007): 402-434.

Rice, Jeff. "Network and New Media." College English 69.2 (Nov 2006): 127-133.

---. The Rhetoric of Cool: Composition Studies and New Media. Southern Illinois UP, 2007.

Run Lola Run. Dir. Tom Tykwer. Sony, 1998.

Rush, Michael. New Media in Late $20^{\text {th }}$ Century Art. London: Thames and Hudson, 1999.

Schodt, Frederik. Dreamland Japan: Writings on Modern Manga. Berkeley: Stone Bridge Press, 1996. 
Schön, Donald A. The Reflective Practitioner: How Professionals Think in Action. New York: Basic Books, 1983.

Scott, Linda M. "Images in Advertising: The Need for a Theory of Visual Rhetoric." Journal of Consumer Research (1994) 21: 252-73.

Selfe, Cynthia. "Lest We Think the Revolution is a Revolution: Images of Technology and the Nature of Change." Passions, Pedagogies and $21^{s t}$ Century Technologies.

Shipka, Jody. “A Multimodal Task-Based Framework for Composing." CCC 57.2 (Dec 2005): 277-306.

Silvio, Carl. "Anime, Both Global and Local." Science Fiction Studies 29.3 (2002 Nov): $489-91$.

Sinatra, Richard. Visual Literacy Connections to Thinking, Reading and Writing. Springfield: Thomas Books, 1986.

Sirc, Geoffrey M. English Composition as a Happening. Logan: Utah State UP, 2002.

---. "Virtual Urbanism." Computers and Composition 18 (2001): 11-19.

Smith, Keri. Wreck This Journal. New York: Perigee, 2007.

Smith, Terry, ed. Impossible Presence: Surface and Screen in the Photogenic Era. Chicago: U of Chicago P, 2001.

Snyder, Ilana. Page to Screen: Taking Literacy into the Electronic Era. London: Routledge, 1998.

Spirited Away. Dir. Hayao Miyazaki. Buena Vista, 2001.

Stephens, Mitchell. The Rise of the Image, the Fall of the Word. New York: Oxford UP, 1998. 
Strunk, William, E.B. White and Maira Kalman. The Elements of Style Illustrated. New York: Penguin, 2007.

Syverson, M. A. "Thinking through Worlds Fair: Evolutionary Rhetoric." Computers and Composition 18 (2001): 163-76.

Takayoshi, Pamela and Brian Huot. "Composing in a Digital World: The Transition of a Writing Program and Its Faculty." WPA: Writing Program Administration 32.2 (Spring 2009):89-119.

---. eds. Teaching Writing with Computers: An Introduction. Boston, MA: HoughtonMifflin, 2003.

Tasker, Yvonne. Spectacular Bodies: Gender, Genre and the Action Cinema. New York: Routledge, 1993.

Trinh, T. Minh ha. Framer/Framed. New York: Routledge, 1992.

Tufte, Edward. Envisioning Information. Cheshire, Conn: Graphics Press, 1990.

Unsworth, Len. Teaching Multiliteracies Across the Curriculum: Changing Contexts of Text and Image in Classroom Practice. Buckingham: Open UP, 2001.

Vitanza, Victor. "Abandoned to Writing: Notes Toward Several Provocations." Enculturation 5.1 (2003).

---. "CompoZing complicating Processes." PRE/TEXT 3/01. 1999-2000.

Warnick, Barbara. "Rhetorical Criticism in New Media Environments." Rhetoric Review 20.1 (2001): 60-65.

Wells, Paul. Understanding Animation. New York: Routledge, 1998.

West, Kanye. “Stronger.” Dir. Hype Williams. Roc-A-Fella Records, 2007. 
Westbrook, Steve. "Visual Rhetoric in a Culture of Fear: Impediments to Multimedia Production." College English 68.5 (May 2006): 457-480.

Williams, Bronwyn. Tuned In: Television and the Teaching of Writing. Portsmouth: Heinemann, 2002.

Williams, Linda, ed. Viewing Positions: Ways of Seeing Film. New Jersey: Rutgers UP, 1994.

Wright, Bradford. Comic Book Nation. Baltimore: Johns Hopkins UP, 2001.

Wysocki, Anne. "Impossibly Distinct: On Form/Content and Word/Image in Two Pieces of Computer-Based Interactive Multimedia." Computers and Composition 18 (2001):137-62.

---. Visual Rhetoric. dated May 13, 2002

http://www.hu.mtu.edu/ awysocki/vizrhet/vr0.html

---. "With Eyes That Think, and Compose, and Think: On Visual Rhetoric." Teaching Writing with Computers. Eds. Pamela Takayoshi and Brian Huot. Boston: Houghton Mifflin, 2003: 182-201.

Wysocki, Anne and Johndan Johnson-Eiola. "Blinded by the Letter: Why Are We Using Literacy as a Metaphor for Everything Else?" Passions, Pedagogies and $21^{s t}$ Century Technologies.

Wysocki, Anne, Johndan Johnson-Eilola, Cynthia L. Selfe and Geoffrey Sirc. Writing New Media: Theory and Applications for Expanding the Teaching of Composition. Logan, Utah: Utah State UP, 2004.

Zettl, H. Sight, Sound, Motion: Applied Media Aesthetics. New York: Wadsworth, 1990. 


\section{APPENDIX}

\section{Afterword: Reflection on Design}

Page:

1 Photo image of blades of grass: free use clip art, standard for Microsoft Word. As described in Chapter 1, one of the design decisions for this dissertation includes using accessible technology.

"Green Fuse" drawn using AutoShapes line tool, curve function: connects and highlights ideas, names, and/or words together. Also inspired by Dylan Thomas" "The Force That Through the Green Fuse Drives the Flower."

The design of the first page serves as a preliminary signal for the visuals that are used throughout the dissertation: parallel discussion, full color images, design details, text boxes and direct audience address.

$7 \quad$ Text box with patterned line format.

10 "Big C, little c composition" image created using WordArt

11 Duchamp Fountain, 1917: www. earlham.edu/ vanbma/20 $\%$ th 20 century/ images/daytwentysix04/htm

Duchamp Bicycle Wheel: www.designboom.com/history/stilllife.html

Jackson Pollack in his studio, photo by Hans Namuth, July 1950:

www.npg.si.edu/img2/namuth/poll3.jpg

Arrows created with AutoShapes line tool, single arrow end

13 Screen capture from The Matrix

14 Screen capture, cropped for size, from Kurosawa's Dreams

15 Cover of Visual Rhetoric in a Digital World:

www.librarything.com/work/479657

Cover of Understanding Comics: http://scottmccloud.com/2-print/index.htiml

16 Scan from Understanding Comics: pages 40-41 
Cover and interior image from The Elements of Style Illustrated:

www.mairakalman.com/elements.html

19 Screen capture from KillBill

20 Computer screen capture of Microsoft Word

Spiral created with AutoShapes line tool, scribble function

21 Computer screen capture of Microsoft Word, cropped to focus on the tool bar

22 Screen capture from Loveless

27 Cover of Picturing Texts: www.picturingtexts.com

Chart from Phelps page 37: re-drawn with text boxes and line tool

28 Multiple overlapping text boxes, some color-only, some including text, integrated with a standard Microsoft Word clip art: frame

30 Cover of Starting Strong: http://store.tcpress.com/0807741329.shtml

Cover of From Another Angle: http://store.tcpress.com/0807739316.shtml

31 Scan from Starting Strong: Plate 1

32 "Geometric Combo" created using AutoShapes rectangle, triangle and oval tools. Square $=50 \%$ transparency; triangle $=75 \%$ transparency; circle $=75 \%$ transparency. These simple shapes are repeated in each chapter; in this grouping they are referred to as a "Geometric Combo."

33 Standard Microsoft Word clip art: frame. Wysocki uses a similarly decorative frame in Writing New Media, page 152.

34 Scan of the cover of Arnheim's Visual Thinking, cropped for size and manipulated using picture "washout" function.

35 Repeat from page 1 "Green Fuse."

Panzani ad from Barthes in color:

http://communication.ucsd.edu/goldfarb/cocu108/data/images/Week2/album/s lides/panzani.html 
39 Panzani ad, Prague 2008: http://marketing-alternativo.es/wpcontent/uploads/2008/10/panzani-marketing-alternativo-es.jpg

42 Circle created using AutoShapes, Oval tool. Cutouts inserted by overlaying AutoShapes, Rectangle tool.

43 AutoShapes Triangle, $60 \%$ transparency.

44 AutoShapes Triangle, $60 \%$ transparency.

45 Magritte This Is Not A Pipe: www.ionoxfordtube.com/01/2009/what-aboutconcretepoetry

Van Gogh Wheatfield with Crows:

http://art.gprc.ab.ca/blogs/modernart/2007/12/17/van-goghs-wheatfield

47 AutoShapes Callout tool, cloud function

48 Scan, cropped for size, from Understanding Comics, page 30

50 Scan from Understanding Comics, page 43

52 Repeat from page 20 Spiral

54 "Green Fuse"

Spiral

55 "Green Fuse"

Spiral

56 Scan of the cover of Mitchell's Iconology

57 Marines logo: http://marines.mil

Text box with AutoShapes triangle overlay

60 Scan of diagram from Multiliteracies page 26

61 Diagram from Multiliteracies page 201, recreated using AutoShapes rectangle and AutoShapes line: combined into one picture using Group function

65 "Geometric Combo"

66 "Geometric Combo"

67 Text box with AutoShapes triangle overlay 
70 Scan from "Rebirth of the Cool" Newtype USA 4.6 (June 2005) page 9

72 Screen capture, cropped for size, from Kurosawa's Dreams

Text box with AutoShapes triangle overlay

73 Women voting AP photo by Vahid Salimi: http://news.yahoo.com/ nphotos/Iran-Election/ss/events/w106/09/09iranelectionss

Rock throwers, AFP photo, 6-20-09: http://news.yahoo.com/nphotos/IranElection/ss/events/w106/09/09iranelectionss

Iranian police, Tazahorate Ma, 6-20-09: http://tazahorate-ma.blogspot.com/ 2009/06/Tehran-6202009.html

$74 \quad$ See photo credit listed for page 73

$75 \quad$ See photo credit listed for page 73

$76 \quad$ See photo credit listed for page 73

Goya The Third of May 1808, public domain image from Wikimedia Commons: http://en.wikipedia.org/wiki/File:Francisco_de_Goya_y_Lucientes -023.jpg

77 Screen captures, cropped for size, from Spirited Away

78 Double text box with AutoShapes overlay: triangle at 39\% transparency, circle at $75 \%$ transparency

79 "Geometric Combo"

80 "Motorcycle lights" created using AutoShapes line tool, curve function: green at 1 pt. size, yellow at 9 pt., red at 4.25 , combined using Group

81 Screen captures: left, Akira; right, video for Kanye West's "Stronger," directed by Hype Williams

82 Screen captures: left, Akira; right, video for Kanye West's "Stronger," directed by Hype Williams

"Red taillight" re-size of the red AutoShape on page 80

83 Screen captures: left, Akira; right, video for Kanye West's "Stronger," directed by Hype Williams 
84 Screen capture from Loveless

85 Screen captures from Loveless

86 Screen captures from Loveless

Text box with AutoShapes triangles, full saturation of color

87 Screen capture from Metropolis

88 Screen capture from Metropolis

89 Screen capture from Metropolis

Screen capture from Grave of the Fireflies

90 Screen capture from Grave of the Fireflies

Text box with AutoShapes rectangles in background

91 AutoShapes rectangles: green at 34\% transparency, blue at $49 \%$ transparency

Screen capture from Cowboy Bebop: The Series opening credits

92 Screen capture from Cowboy Bebop: The Series opening credits

93 Screen capture from Cowboy Bebop: The Series opening credits

95 "Geometric Combo"

99 Standard Microsoft Word clip art: recycling box

Duchamp The Green Box: http://inedandunlined.com/2007/08/28/collectedwords

100 Duchamp Box in a Valise: http://artsearch.nga.gov.au.Detail.cfm?IRN=64922 \&PICTAUS $=$ TRUE

101 Scan of page of student composition, Fall 2002, University of Louisville

105 "Geometric Combo"

107 AutoShapes triangle at $60 \%$ transparency 
108 Scan from "Visual Vision: Susan Howe's 'The Nonconformist's Memorial' written for Alan Golding's Eng 665, April 1998

109 Scans from student essay, donated to Ware teaching files

$110 \quad$ Scan from Berger's Ways of Seeing page 38

Scan from student essay

112 Scan from student essay

113 Screen capture from Metropolis

115 Scans from Bang's Picture This pages 35, 37

116 Scan from Bang's Picture This page 133

117 Crop of scan from Bang's Picture This page 133

118 AutoShapes separated and resized from "Geometric Combo": square at 50\% transparency, triangle at $75 \%$ transparency, circle at $75 \%$ transparency

119 Storyboard sample, Hanna Barbera: http://cartoonsanap.blogspot.com/2008/12 /storyboards-huckleberry-hound-in.html

120 Scans from McCloud's 24 Hour Comics: Davison "The Invisible Library" page 197; Lasky "Minutiae" page 156

121 Post-It Note art by Mare Johns: www.marcjohns.com

122 Post-It Note art by Marc Johns: www.marcjohns.com

123 Mind map by Austin Kleon: www.austinkleon.com

Photo of Sunni Brown with an in progress graphic recording: http://sunni Brown.com/category/graphic-recording

125 "Green fuse"

126 "Geometric Combo" and AutoShapes spiral

127 Text box with Microsoft Word clip art and WordArt overlay

"Green fuse"

128 Text box with AutoShapes triangle overlay at $75 \%$ transparency 
129 Screen captures from Cowboy Bebop: The Movie. Multiple text boxes, one in blue line offset to visually free-associate the idea of "spectacle"

130 Screen capture from The Animatrix

131 Screen capture from Ghost in the Shell

Cropped scan from Berger's Ways of Seeing page 38

AutoShapes triangle at $49 \%$ transparency

132 "Geometric Combo"

133 Scan of student essay (also on page 112)

Cover of Smith's Wreck This Journal: www.kerismith.com

Wreck This Journal completed interior by Jennifer Suarez from amazon.com customer images: www.amazon.com/gp/customer-media/productgallery $/ 039953346 \mathrm{X} / \mathrm{ref}=\mathrm{cm} \_$ciu_pdp_images_1?ie $=U T F 8 \&$ index $=1$

134 Interior of one of the 1,000 journals in the 1000 Journals Project: http://1000journals.com

135 Cover of one of the 1,000 journals in the 1000 Journals Project: http://1000journals.com

138 "Big C, little c composition" image created using WordArt resized from page 10

"Big S, little s subject" image created using WordArt

139 Homage to Berger's Ways of Seeing page 166 


\section{CURRICULUM VITAE}

\section{Karen Ware}

215 W. Burnett Ave. \#3

Louisville, KY 40208

502.637 .9441 home

502.489 .0936 cell

karen@atlyss.com

\section{EDUCATION}

\section{University of Louisville}

Ph.D. in Rhetoric and Composition August 2009

Dissertation: "Ultrakinetic Anime Texts: Revisioning Composition Theory and Exploring Visual Rhetoric Pedagogy"

MA in Literature August 2001

Thesis: "Frank O'Hara's Oranges: Poetry, Painters and Painting"

Spalding University

BA in Psychology and English, minor in Art History

Magna Cum Laude May 1994

Birkbeck College, University of London

Exeter College, Oxford University

Summer Study in English Literature 1993

Presentation Academy, Louisville, KY

$3^{\text {rd }}$ in class May 1991

\section{EXPERIENCE}

Atlyss, LLC

Client Services and Product Development

Fall 2006 - Present

Joined Atlyss fulltime in October of 2006, after participating on a part-time basis in the validation of the Atlyss Operating Style Assessment. Direct advisor to clients regarding the analysis of Atlyss assessment results and subsequent application of Atlyss philosophy.

Tourism Honors Academy

Program Advisor

Spring 2005 - Present

Provide administration, oversight and assistance to an academic honors program for high school students who have overcome adversity in order to achieve. The program features a 10-day summer residential session followed by feature events throughout the Senior year of the scholars. The goal is to expose bright students from diverse backgrounds and experiences to opportunities in an industry they 
Spalding University

might otherwise not consider. My participation in this program includes all stages: from the initial concept to inception to current operation.

Adjunct Professor

Fall 2004 - Present

Courses taught:

Eng 340/540: The Teaching of Writing

Kentucky Governor's Scholars Program

2006

Grant Writing Specialist and Writing Coordinator Summer 1999 - Fall

Primary writer and editor for documents, letters, communications, handbooks, reports, and grant submissions for this public/private partnership. Also assisted in program administration, including special initiatives, such as the former Effective Learning Program at Ballard High School for at-risk students.

University of Louisville

Assistant Director of Composition

Fall 2002 - Spring 2004

Served as a graduate student administrator. Responsibilities included scheduling of all sections of Composition each semester, coordination with Computer Assisted Instruction, evaluation of English transfer credits, administration of professional development workshops, student and instructor grievances and mentoring of peer teaching groups.

Literacy, Technology and Education Teaching Intern

Spring 2004

Co-taught Eng 687 graduate seminar at University of Louisville with Thomas R. Watson Visiting Professor Cynthia Selfe of Michigan Technological University. Internship included extensive management of technology equipment, including digital audio and video recorders.

Graduate Teaching Assistant, English Composition

Fall 1997 - Spring 2004

Courses taught:

Eng 101: Introduction to College Writing

Eng 102: Intermediate College Writing

Eng 105: Advanced Composition for Freshmen

Eng 309: Advanced Academic Writing

Composition)

Eng 602: Teaching College Composition (team taught with Director of

Instructor and Team Assistant

Fall 1999 - Spring 2002

Taught First-Year Composition in the Effective Learning Program, an initiative

for at-risk students. Program structure included linked courses in English, Math, History, and General Studies. Assisted with program coordination, administration and assessment.

Summer 1997

Graduate Assistant, Office of the President $\quad$ Summer 1996 -

Responsibilities included writing, editing and proofreading of correspondence, speeches and various publications pertaining to the Office and the President. Also served as Special Assistant to the Board of Overseers, the University Grievance Committee, and the Metro United Way Fund Drive. 
Conference on College Composition and Communication 2004, San Antonio

"The XX-Files: Tales of Resistance to Female Authority" with Marcy Tucker

$20^{\text {th }}$ Century Literature Conference 2004, University of Louisville

"Identity, Social Hierarchies, and Technological Dangers: Postmodern Elements in the Metropolis"

Image and Imagery Conference 2002, Brock University (Toronto, Ontario, Canada)

"Frank O'Hara's Oranges: Poetry, Painters and Painting"

Thomas R. Watson Conference 2002, University of Louisville

"Vulnerable Identities: Assessment, Resistance and Acceptance in At-Risk Students"

New Voices 2001, Georgia State University

"Toward a Theory of Effective Learning: Composition Classes and Pedagogy in a Pilot Program for At-Risk First-Year Students"

\section{PUBLICATIONS}

"Frank O'Hara's Oranges: The Visual Rhetoric of Poetry, Painters and Painting." Image and Imagery. Ed. Corrado Frederici. New York: Peter Lang, 2005.

"The Road to Mainstreaming: One Program's Successful but Cautionary Tale." with Anthony Edgington, Brian Huot and Marcy Tucker. Discord and Direction. Eds. Sharon James McGee and Carolyn Handa. Logan, Utah: Utah State UP, 2005.

"Teaching New Instructors to Comment: Reading, Responding, and Reflecting" with Anthony Edgington, Brian Huot and Stacy Taylor. Bootcamp. Ed. Sid Dobrin. NCTE [forthcoming]

\section{Affiliations and Distinctions}

Member, National Council of Teachers of English (NCTE)

Member, University of Louisville English Graduate Organization

Chair, Twentieth Century Literature Conference 1998, 1999, 2000, 2004

University of Louisville Student Athlete Faculty Mentor 1998

Alumni Member, Psi Chi, the National Honor Society in Psychology 